\title{
Para-Selective Gold-Catalyzed Direct Alkynylation of Anilines
}

\author{
Jonathan P. Brand and Jérôme Waser* \\ Laboratory of Catalysis and Organic Synthesis, Institute of Chemical Sciences and \\ Engineering, Ecole Polytechnique Fédérale de Lausanne, EPFL SB ISIC LCSO, BCH \\ 4306, 1015 Lausanne, Switzerland.
}

jerome.waser@epfl.ch

Received Date (will be automatically inserted after manuscript is accepted)

A method for the para selective alkynylation of anilines is reported using $\mathrm{AuCl}$ as catalyst and triisopropylsilylethynyl-1,2-benziodoxol-3(1H)-one (TIPS-EBX) as an electrophilic acetylene equivalent. Para-alkynyl anilines substituted at positions 2 or 3 were obtained in one step from simple anilines under mild conditions (room temperature to $60^{\circ} \mathrm{C}$ ) under air. The methodology could also be extended to the alkynylation of trimethoxybenzenes.

Heteroarylacetylenes are important structures in both organic synthesis and material sciences. ${ }^{1}$ They are versatile building blocks thanks to the large number of transformations available based on the functionalization of the triple bond. In addition, both aromatics and acetylenes have been successfully used in extended $\pi$ systems for organic electronic materials. ${ }^{2}$ In order to access heteroarylacetylenes, the Sonogashira reaction is one of the most popular methods for $\mathrm{sp}^{2}$-sp bond

(1) Diederich F.; Stang, P. J.; Tykwinski, R. R.; Acetylene Chemistry: Chemistry, Biology and Material Science; Wiley-VCH, Weinheim, 2005.

(2) (a) Tour, J. M. Acc. Chem. Res. 2000, 33, 791. (b) Tour, J. M. Chem. Rev. 1996, 96, 537. (c) Moore, J. S. Acc. Chem. Res. 1997, 30, 402. (d) Haley, M. M.; Pak, J. J.; Brand, S. C. Top. Curr. Chem. 1999, 201, 81. (e) Youngs, W. J.; Tessier, C. A.; Bradshaw, J. D. Chem. Rev. 1999, 99, 3153. (f) Haley, M. M. Synlett 1998, 557. (g) Pinto, M. R.; Schanze, K. S. Synthesis 2002, 1293. (h) Yamamoto, T. Synlett 2003 425. (i) Tobe, Y.; Sonoda, M. In Modern Cyclophane Chemistry; Gleiter, R., Hopf, H., Eds.; Wiley VCH: Weinheim, 2004; p 1. (j) Swager T.M.; Semiconducting Poly(arylene ethylene)s in Acetylene Chemistry: Chemistry, Biology and Material Science; Diederich, F.; Stang, P. J.; Tykwinski, R. R. ed.; Wiley-VCH, Weinheim, 2005. (k) James, D. K.; Tour, J. M. Top. Curr. Chem. 2005, 257, 33. formation. ${ }^{3}$ However, the main drawback of this method is the required prefunctionnalization of the $\mathrm{sp}^{2}$ carbon. Numerous methods have been developed in the field of $\mathrm{C}-\mathrm{H}$ arylation in order to avoid this prefunctionalization of aromatics. $^{4}$ Surprinsingly, reports of direct alkynylations were scarce up to $2009 . .^{5}$ Since then, important breakthroughs have been realized and several groups reported new C-H alkynylation methods. ${ }^{6}$ Our

(3) (a) Sonogashira, K.; Tohda, Y.; Hagihara, N. Tetrahedron Lett. 1975, 16, 4467. (b) Sonogashira, K. In Handbook of Organopalladium Chemistry for Organic Synthesis; Negishi, E., Ed.; Wiley: New York, 2002; p 493. (c) Sonogashira, K. J. Organomet. Chem. 2002, 653.

(4) (a) Alberico, D.; Scott, M. E.; Lautens, M. Chem. Rev. 2007, 107, 174. (b) Seregin, I. V.; Gevorgyan, V. Chem. Soc. Rev. 2007, 36, 1173. (c) Bellina, F.; Rossi, R. Tetrahedron 2009, 65, 10269. (d) Ackermann, L.; Vicente, R.; Kapdi, A. R. Angew. Chem., Int. Ed. 2009, 48, 9792.

(5) (a) Kobayashi, K.; Arisawa, M.; Yamaguchi, M. J. Am. Chem. Soc. 2002, 124, 8528. (b) Amemiya, R.; Fujii, A.; Arisawa, M.; Yamaguchi, M. J. Organomet. Chem. 2003, 686, 94. (c) Seregin, I. V.; Ryabova, V.; Gevorgyan, V. J. Am. Chem. Soc. 2007, 129, 7742. For non catalytic methods, see: (d) Trofimov, B. A.; Stepanova, Z. V.; Sobenina, L. N.; Mikhaleva, A. I.; Ushakov, I. A. Tetrahedron Lett. 2004, 45, 6513. (e) Kalinin, V. N.; Pashchenko, D. N.; She, F. M. Mendeleev Commun. 1992, 60.

(6) Reviews: (a) Dudnik, A. S.; Gevorgyan, V. Angew. Chem. Int., $E d$. 2010, 49, 2096. (b) Messaoudi, S.; Brion, J. D.; Alami, M. Eur. J. Org. Chem. 2010, 6495. Selected examples: (c) Matsuyama, N.; Hirano, 
group especially focused on the functionalization of electron-rich heterocycles such as indoles, pyrroles and thiophenes using triisopropylsilylethynyl-1,2benziodoxol-3(1H)-one (TIPS-EBX (1)) as electrophilic alkynylation reagent and $\mathrm{AuCl}$ as catalyst. ${ }^{7}$ The focus on the synthesis of silylated acetylenes is motivated by their simple deprotection to access the synthetically highly versatile terminal alkynes.

Presently, there are only two reports for the alkynylation of anilines by Yamaguchi and Chatani, ${ }^{5 b, 6 j}$ with both methods affording exclusively ortho alkynylated products. This selectivity was rationalized by a mechanism involving a directing effect of the nitrogen functional group. As the gold-catalyzed alkynylation did not require a directing group, we hypothesized that para selective functionalization could be realized (Scheme 1). Herein, we report the development of the first paraselective alkynylation of anilines using TIPS-EBX (1) as acetylene-transfer reagent, which proceeds under mild conditions (room temperature to $60{ }^{\circ} \mathrm{C}$, ambient atmosphere).

Scheme 1. Direct Alkynylation of Anilines

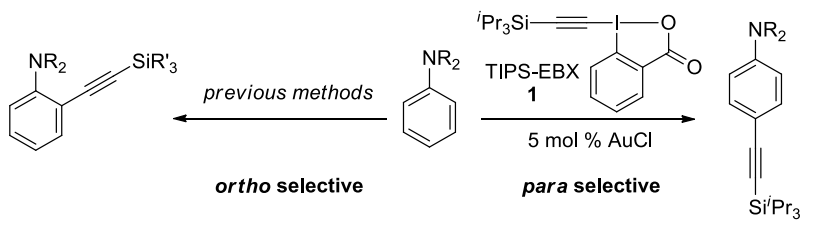

Para-alkynyl anilines are widely used in material sciences, especially as strong electron donors in push-pull chromophores for applications in optoelectronic devices (Figure 1). ${ }^{8}$ For example, tetraalkyne 2 has shown easily tunable photochromic properties. ${ }^{8 \mathrm{f}}$ The tetraethynylene $\mathbf{3}$

K.; Satoh, T.; Miura, M. Org. Lett. 2009, 11, 4156. (d) Kawano, T.; Matsuyama, N.; Hirano, K.; Satoh, T.; Miura, M. J. Org. Chem. 2010 75, 1764. (e) Kitahara, M.; Hirano, K.; Tsurugi, H.; Satoh, T.; Miura, M. Chem. Eur. J. 2010, 16, 1772. (f) Matsuyama, N.; Kitahara, M.; Hirano, K.; Satoh, T.; Miura, M. Org. Lett. 2010, 12, 2358. (g) Besselievre, F.; Piguel, S. Angew. Chem., Int. Ed. 2009, 48, 9553. (h) Berciano, B. P.; Lebrequier, S.; Besselievre, F.; Piguel, S. Org. Lett. 2010, 12, 4038. (i) Kim, S. H.; Chang, S. Org. Lett. 2010, 12, 1868. (j) Tobisu, M.; Ano, Y.; Chatani, N. Org. Lett. 2009, 11, 3250. (k) Gu, Y. H.; Wang, X. M. Tetrahedron Lett. 2009, 50, 763. (1) de Haro, T.; Nevado, C. J. Am. Chem. Soc. 2010, 132, 1512. (m) Yang, L.; Zhao, L. A.; Li, C. J. Chem. Commun. 2010, 46, 4184

(7) (a) Brand, J. P.; Charpentier, J.; Waser, J. Angew. Chem., Int. Ed. 2009, 48, 9346. (b) Brand, J. P.; Waser, J. Angew. Chem., Int. Ed. 2010, 49, 7304. (c) Brand, J. P.; Chevalley, C.; Waser, J. Beilstein J. Org. Chem. 2011, 7, 565. First synthesis of TIPS-EBX (1): (d) Zhdankin, V. V.; Kuehl, C. J.; Krasutsky, A. P.; Bolz, J. T.; Simonsen, A. J. J. Org. Chem. 1996, 61, 6547.

(8) Selected examples: (a) Kivala, M.; Diederich, F. Acc. Chem. Res. 2009, 42, 235. (b) Gobbi, L.; Elmaci, N.; Luthi, H. P.; Diederich, F. ChemPhysChem 2001, 2, 423. (c) Bosshard, C.; Spreiter, R.; Gunter, P.; Tykwinski, R. R.; Schreiber, M.; Diederich, F. Adv. Mater. 1996, 8, 231. (d) Gobbi, L.; Seiler, P.; Diederich, F. Angew. Chem., Int. Ed. 1999, 38, 674. (e) Reutenauer, P.; Kivala, M.; Jarowski, P. D.; Boudon, C.; Gisselbrecht, J. P.; Gross, M.; Diederich, F. Chem. Commun. 2007, 4898. (f) Marsden, J. A.; Miller, J. J.; Shirtcliff, L. D.; Haley, M. M. J. Am. Chem. Soc. 2005, 127, 2464.
(TEES) has molecular photoswitch properties. $^{8 \mathrm{~d}}$ Furthermore, ethynylanilines are used as starting materials for the synthesis of chromophores based on core structure 4 via [2+2]-cycloaddition with tetracyanoethene followed by retro-electrocyclization. ${ }^{8 \mathrm{e}}$ Consequently, an efficient access to para alkynylated anilines would lead to a more straightforward synthesis of electronic organic materials.

Figure 1. Para-Alkynyl Anilines in Material Sciences.

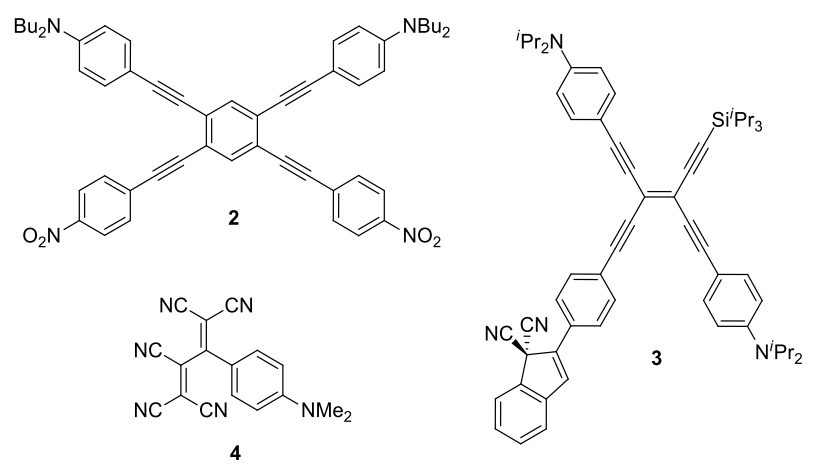

Gold catalysis has recently been investigated for the direct functionalization of benzene rings via amination, ${ }^{9}$ alkylation, ${ }^{10}$ hydroarylation ${ }^{11}$ and arylation. ${ }^{12}$ Only one example of the catalytic use of gold for the alkynylation of benzene rings has been reported, but no anilines were used in that work. ${ }^{61,13}$ Moreover, there are only two single examples of gold-catalyzed direct functionalization of anilines for amination ${ }^{9 \mathrm{~b}}$ and hydroarylation. ${ }^{11 \mathrm{~d}}$ Consequently, the extension of the alkynylation reaction to anilines would constitute an important advance, not only in the field of acetylene chemistry, but also for gold catalysis in general, especially in light of nitrogencontaining groups being reported to deactivate gold catalysts in several cases. ${ }^{14}$

In order to investigate the alkynylation of protected anilines with TIPS-EBX (1), ${ }^{15}$ we decided to use $N, N$ -

(9) (a) Li, Z. G.; Capretto, D. A.; Rahaman, R. O.; He, C. J. Am. Chem. Soc. 2007, 129, 12058. (b) Gu, L.; Neo, B. S.; Zhang, Y. Org. Lett. 2011, 13, 1872.

(10) Shi, Z. J.; He, C. J. Am. Chem. Soc. 2004, 126, 13596

(11) Selected examples: (a) Reetz, M. T.; Sommer, K. Eur. J. Org. Chem. 2003, 3485. (b) Shi, Z. J.; He, C. J. Org. Chem. 2004, 69, 3669. (c) Reich, N. W.; Yang, C. G.; Shi, Z. J.; He, C. Synlett 2006, 1278. (d) Jean, M.; van de Weghe, P. 2011, 52, 3509.

(12) Kar, A.; Mangu, N.; Kaiser, H. M.; Beller, M.; Tse, M. K. Chem. Commun. 2008, 386.

(13) For the stoichiometric use of gold, see: Fuchita, Y.; Utsunomiya, Y.; Yasutake, M. J. Chem. Soc. Dalton Trans. 2001, 2330.

(14) (a) Belot, S.; Vogt, K. A.; Besnard, C.; Krause, N.; Alexakis, A., Angew. Chem., Int. Ed. 2009, 48, 8923. (b) Monge, D.; Jensen, K. L.; Franke, P. T.; Lykke, L.; Jorgensen, K. A., Chem. Eur. J. 2010, 16, 9478. (c) Loh, C. C. J.; Badorrek, J.; Raabe, G.; Enders, D. Chem. Eur. J. 2011, 17, 13409 .

(15) Our work has been focused on TIPS-EBX (1) to afford easy to deprotect silyl acetylenes. Furthermore, a bulky silyl group was shown to be essential for the success of the reaction. See reference 7 . Indeed, no product was obtained using phenylethynyl-1,2-benziodoxol-3(1H)one as acetylene transfer reagent. 
benzylaniline (5a) as model compound since it is more nucleophilic than the corresponding carbamate and the benzyl groups are easily removed by hydrogenation. Unfortunately, the reaction conditions previously optimized for indoles ${ }^{7 a}$ only afforded a $14 \%$ yield employing $N, N$-dibenzylaniline (5a) as substrate (Table 1, entry 1) due to low conversion. The outcome of the reaction was highly dependent on the solvent, with ${ }^{i} \mathrm{PrOH}$ giving the best result (entries 2-4). No ortho alkynylation was observed for aniline 5a. The use of 1.4 equivalent of TIPS-EBX (1) was optimal (entries 4-6). The use of a higher concentration did not improve the yield (entry 7). Best results were obtained when the reaction was not pushed to full conversion in order to prevent the formation of side products. In this case, the starting material could easily be recovered. The obtained yields are in the same range as those obtained in the current state-of-the-art direct para-functionalizations of anilines. $^{16}$

Table 1. Optimization of the para-Alkynylation of Anilines.

Table 1. Optimization of the para-Alkynylation of Anilines.

\begin{tabular}{|c|c|c|c|}
\hline & $5 \mathrm{~mol}$ & \multicolumn{2}{|l|}{6} \\
\hline entry & solvent & $\begin{array}{c}\text { TIPS-EBX (1) } \\
\text { equivalents }\end{array}$ & yield $^{\mathrm{a}}$ \\
\hline 1 & $\mathrm{Et}_{2} \mathrm{O}$ & 1.2 & $14 \%$ \\
\hline 2 & $\mathrm{CH}_{2} \mathrm{Cl}_{2}$ & 1.2 & $49 \%$ \\
\hline 3 & $\mathrm{CH}_{3} \mathrm{CN}$ & 1.2 & $51 \%$ \\
\hline 4 & ${ }^{i} \mathrm{PrOH}$ & 1.2 & $58 \%$ \\
\hline 5 & ${ }^{i} \mathrm{PrOH}$ & 1.4 & $73 \%(84 \%)^{\mathrm{b}}$ \\
\hline 6 & ${ }^{i} \mathrm{PrOH}$ & 1.6 & $55 \%$ \\
\hline $7^{\mathrm{c}}$ & ${ }^{i} \mathrm{PrOH}$ & 1.4 & $68 \%(81 \%)^{\mathrm{b}}$ \\
\hline \multicolumn{4}{|c|}{$\begin{array}{l}{ }^{a} \text { Reaction conditions: } 0.20 \mathrm{mmol} N, N \text {-dibenzylaniline }(\mathbf{5 a}), 0.01 \\
\text { mmol AuCl in } 4 \mathrm{~mL}{ }^{i} \mathrm{PrOH} \text { at } 23{ }^{\circ} \mathrm{C} \text { under air. Isolated yield after } \\
\text { column chromatography. }{ }^{\mathrm{b}} \text { Yield based on recovered starting material } \\
\text { (brsm). }{ }^{\mathrm{c}} 2 \mathrm{~mL}{ }^{i} \mathrm{PrOH} \text {. }\end{array}$} \\
\hline
\end{tabular}

We then focused our attention on the scope of the reaction (Table 2). In addition to benzyl (entry 1), butyl, ethyl and methyl groups were tolerated as nitrogen substituents, but led to lower yields (entries 2-4). Smaller alkyl groups led to a complex mixture of side products, including $8 \%$ of the ortho-para disubstituted product for $\mathrm{R}=\mathrm{Me}$ (entry 4). These results indicate that the process has the regioselectivity of an aromatic electrophilic substitution. It is in line with our previous highly SEAr regioselective methodologies for the alkynylation of indoles, pyrroles and thiophenes. ${ }^{7 \mathrm{a}, \mathrm{b}}$

Ortho methyl, phenyl and methoxy groups were tolerated for monoprotected anilines (entries 5-7). ${ }^{17}$ This result also demonstrated that the method was tolerant towards a free NH bond on the aniline. The alkynylation

(16) Ciana, C. L.; Phipps, R. J.; Brandt, J. R.; Meyer, F. M.; Gaunt, M. J. Angew. Chem., Int. Ed. 2011, 50, 458.

(17) Ortho substituted $N, N$-disubstituted anilines were not reactive, probably because of the deconjugation of the nitrogen for steric reasons. reaction was even successful in the case of the less reactive dibenzyl 2-aminonaphthalene $(\mathbf{5 h})$, although full conversion could not be achieved in this case (entry 8).

Table 2. Scope of the para-Alkynylation of Anilines.

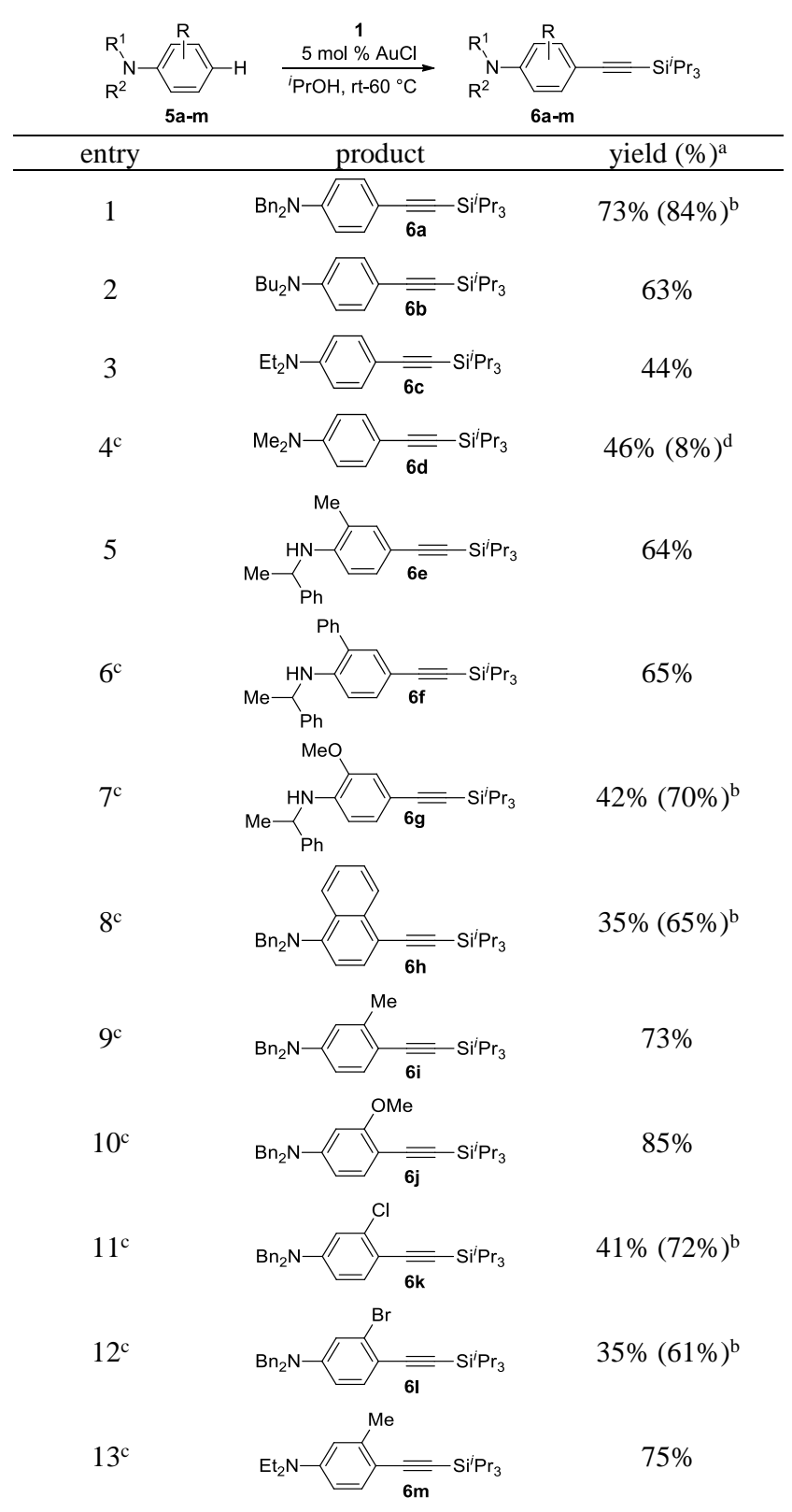

Reaction conditions: $0.40 \mathrm{mmol} \mathbf{5}, 0.56 \mathrm{mmol} \mathbf{1}, 0.02 \mathrm{mmol} \mathrm{AuCl}$ in 8 $\mathrm{mL}{ }^{i} \mathrm{PrOH}$ at $23{ }^{\circ} \mathrm{C}$ under air. Isolated yields. ${ }^{\text {b}}$ Yields based on recovered starting materials. ${ }^{c}$ at $60{ }^{\circ} \mathrm{C}$ in $2 \mathrm{~mL}{ }^{i} \mathrm{PrOH}$. ${ }^{\mathrm{d}}$ ortho-para disubstituted product.

Importantly, alkynylation was also possible for alkyl, methoxy, chloro and bromo groups in the meta position, giving more sterically hindered 1,3,4-substituted anilines (entries 9-13). In addition, the tolerance to chloro and bromo substituents demonstrated the orthogonality of the method to classical Pd cross-couplings. Low yields of ortho alkynylated products were obtained using para 
substituted anilines. ${ }^{18}$ In the case of 4-methyl- $N, N$ dimethyl aniline (5n), one of the main products obtained was the $\mathrm{sp}^{3}$ coupling product on the methyl substituent of the nitrogen (eq 1). It is interesting to note that this reaction can easily be achieved with free acetylenes and metal catalysts under oxidative conditions. ${ }^{19}$ Consequently, it could indicate that this product results from the relatively high oxidation capacity of TIPS-EBX (1).

$$
\text { Me }
$$
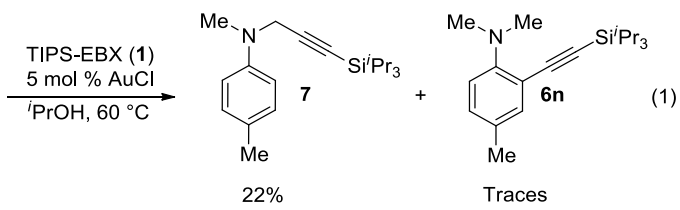

In addition to anilines, the reaction could also be applied towards the alkynylation of trimethoxybenzenes (Figure 2). Up to now, the only report for the alkynylation of this class of substrates was limited to the use of more electron-deficient propiolic acid derivatives. ${ }^{61}$ The use of TIPS-EBX (1) allowed access to an easily deprotectible non electron-deficient acetylene.

Figure 2. Alkynylation of trimethoxybenzenes ${ }^{\mathrm{a}}$

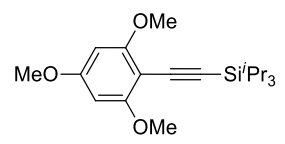

8 a, $51 \%(80 \%)$

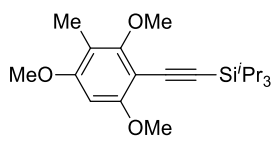

8 b, $50 \%(79 \%)$

a Reaction conditions: $0.40 \mathrm{mmol}$ trimethoxybenzene, $0.56 \mathrm{mmol} \mathbf{1}, 0.02$ $\mathrm{mmol} \mathrm{AuCl}$ in $2 \mathrm{~mL}{ }^{i} \mathrm{PrOH}$ at $60{ }^{\circ} \mathrm{C}$ under air. Isolated yields. Yields based on recovered starting materials under parenthesis.

In conclusion, we have developed the first paraselective direct alkynylation of anilines using gold chloride as catalyst and TIPS-EBX (1) as acetylenetransfer reagent. The method allowed a new and efficient access to important building blocks in synthetic chemistry and material sciences under mild reaction conditions and at ambient atmosphere. We demonstrated that a wide range of anilines could be alkynylated regioselectively using the developed methodology. Investigations towards the elucidation of the reaction mechanism are currently ongoing in our laboratory.

Supporting Information. Experimental procedures, analytical data for all new compounds. This material is

(18) 4-Methyl- $N, N$-dibenzylaniline was ortho alkynylated in $18 \%$ yield.

(19) For $\mathrm{sp}^{3}$ alkynylation of dimethylaniline (5d), see: (a) Li, Z. P.; Li, C. J. J. Am. Chem. Soc. 2004, 126, 11810. (b) Volla, C. M. R.; Vogel, P. Org. Lett. 2009, 11, 1701. (c) Liu, P.; Zhou, C. Y.; Xiang, S.; Che, C. M. Chem. Commun. 2010, 46, 2739. For cyanation of dimethylaniline (5d) using hypervalent iodine, see: (d) Zhdankin, V. V.; Kuehl, C. J.; Krasutsky, A. P.; Bolz, J. T.; Mismash, B.; Woodward, J. K.; Simonsen, A. J. Tetrahedron Lett. 1995, 36, 7975. For $\mathrm{sp}^{3}$ cyanation of dimethylaniline (5d) using a gold complexe, see: (e) Zhang, Y.; Peng, H.; Zhang, M.; Cheng, Y. X.; Zhu, C. J. Chem. Commun. 2011, 47, 2354. 


\title{
Supporting Information
}

\section{Para-Selective Gold-Catalyzed Direct Alkynylation of Anilines}

\author{
Jonathan P. Brand and Jérôme Waser.* \\ Laboratory of Catalysis and Organic Synthesis, Institute of Chemical Sciences and Engineering, Ecole Polytechnique \\ Fédérale de Lausanne, EPFL SB ISIC LCSO, BCH 4306, 1015 Lausanne, Switzerland.
}

38 pages

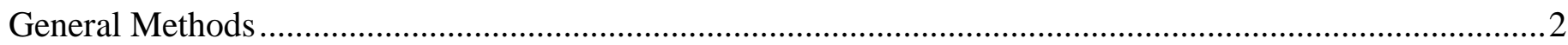

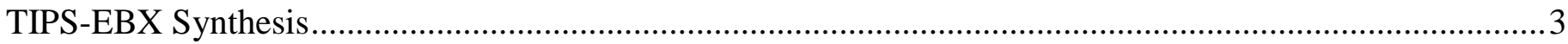

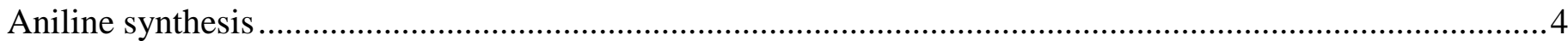

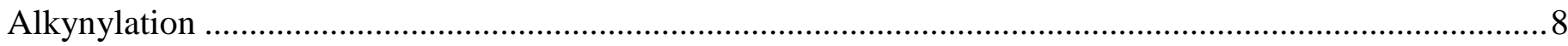

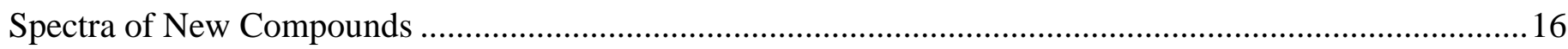




\section{General Methods}

All reactions were carried out in oven dried glassware under an atmosphere of nitrogen, unless stated otherwise. For quantitative flash chromatography technical grade solvents were used. For flash chromatography for analysis, HPLC grade solvents from Sigma-Aldrich were used. THF, $\mathrm{Et}_{2} \mathrm{O}, \mathrm{CH}_{3} \mathrm{CN}_{\text {, }}$ toluene, hexane and $\mathrm{CH}_{2} \mathrm{Cl}_{2}$ were dried by passage over activated alumina under nitrogen atmosphere $\left(\mathrm{H}_{2} \mathrm{O}\right.$ content $<10 \mathrm{ppm}$, Karl-Fischer titration). $\mathrm{NEt}_{3}$ and pyridine were distilled under nitrogen from KOH. Gold chloride was purchased from Aldrich or Alfa Aesar and kept in desiccator under anhydrous condition (decrease of reactivity has been observed for catalyst if prolonged exposition to air ( $\mathrm{ca} 1 \mathrm{month}$ ). All chemicals were purchased from Acros, Aldrich, Fluka, VWR, Aplichem or Merck and used as such unless stated otherwise. Chromatographic purification was performed as flash chromatography using MachereyNagel silica 40-63, $60 \AA$, using the solvents indicated as eluent with 0.1-0.5 bar pressure. TLC was performed on Merck silica gel $60 \mathrm{~F}_{254}$ TLC glass plates or aluminium plates and visualized with UV light, permanganate stain, CAN stain or anisaldehyde stain. Melting points were measured on a calibrated Büchi B-540 melting point apparatus using open glass capillaries. ${ }^{1} \mathrm{H}-\mathrm{NMR}$ spectra were recorded on a Brucker DPX-400 $400 \mathrm{MHz}$ spectrometer in chloroform-d, $\mathrm{C}_{6} \mathrm{D}_{6}$ DMSO- $\mathrm{d}_{6}$ or $\mathrm{CD}_{3} \mathrm{OD}$, all signals are reported in ppm with the internal chloroform signal at $7.26 \mathrm{ppm}$, the internal $\mathrm{C}_{6} \mathrm{D}_{6}$ signal at $7.16 \mathrm{ppm}$. the internal DMSO signal at $2.50 \mathrm{ppm}$ or the internal methanol signal at $3.30 \mathrm{ppm}$ as standard. The data is being reported as $(\mathrm{s}=$ singlet, $\mathrm{d}=$ doublet, $\mathrm{t}=$ triplet, $\mathrm{q}=$ quadruplet, $\mathrm{qi}=$ quintet, $\mathrm{m}=$ multiplet or unresolved, br = broad signal, app = apparent, coupling constant(s) in Hz, integration, interpretation). ${ }^{13} \mathrm{C}$ NMR spectra were recorded with ${ }^{1} \mathrm{H}$-decoupling on a Brucker DPX-400 $100 \mathrm{MHz}$ spectrometer in chloroform-d, DMSO- $\mathrm{d}_{6}$ or $\mathrm{CD}_{3} \mathrm{OD}$, all signals are reported in ppm with the internal chloroform signal at $77.0 \mathrm{ppm}$, the internal DMSO signal at $39.5 \mathrm{ppm}$ or the internal methanol signal at $49.0 \mathrm{ppm}$ as standard. Some benziodoxol(on)es NMRs in $\mathrm{CDCl}_{3}$ showed a small dependence to the concentration. As a result, the concentration of the corresponding NMR samples is indicated. Infrared spectra were recorded on a JASCO FT-IR B4100 spectrophotometer with an ATR PRO410-S and a ZnSe prisma and are reported as $\mathrm{cm}^{-1}(\mathrm{w}=$ weak, $\mathrm{m}=$ medium, $\mathrm{s}=$ strong, $\mathrm{br}=$ broad). Gas chromatographic and low resolution mass spectrometric measurements were performed on a Perkin-Elmer Clarus 600 gas chromatographer and mass spectrometer using a Perkin-Elemer Elite fused silica column (length: $30 \mathrm{~m}$, diameter: $0.32 \mathrm{~mm}$ ) and Helium as carrier gas. High resolution mass spectrometric measurements were performed by the mass spectrometry service of ISIC at the EPFL on a MICROMASS (ESI) Q-TOF Ultima API. HPLC measurement were done on a JASCO HPLC system with an AS2055 Autosampler, a PU 2089 Pump, a UV 2075 detector and a SEDEX 85 (SEDERE) detector using a CHIRALPAK IC column from DAICEL Chemical Industries Ltd. HPLC grade solvents from Sigma-Aldrich were used. 


\section{TIPS-EBX Synthesis}

Triisopropylsilyl trimethylsilylacetylene (10)

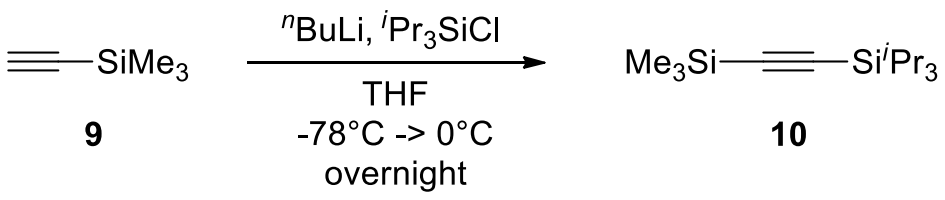

Following a reported procedure, ${ }^{1} n$-butyllithium $(2.5 \mathrm{M}$ in hexanes, $12.0 \mathrm{~mL}, 29.9 \mathrm{mmol}, 0.98$ equiv) was added dropwise to a stirred solution of ethynyltrimethylsilane (9) (3.0 g, $30 \mathrm{mmol}, 1.0$ equiv) in THF (48 $\mathrm{mL}$ ) at $-78{ }^{\circ} \mathrm{C}$. The mixture was then warmed to $0{ }^{\circ} \mathrm{C}$ and stirred for $5 \mathrm{~min}$. The mixture was then cooled back to $-78{ }^{\circ} \mathrm{C}$ and chlorotriisopropylsilane $(6.4 \mathrm{~mL}, 30 \mathrm{mmol}, 1.0$ equiv) was added dropwise. The mixture was then allowed to warm to room temperature and stirred overnight. A saturated solution of ammonium chloride $(40 \mathrm{~mL})$ was added, and the reaction mixture was extracted with diethyl ether $(2 \times 60 \mathrm{~mL})$. The organic layer was washed with water and brine, then dried over $\mathrm{MgSO}_{4}$, filtered and concentrated under reduced pressure to obtain a colorless liquid which was further purified by Kugelrohr distillation (56$57^{\circ} \mathrm{C} / 0.25 \mathrm{mmHg}$ ) to yield $10\left(7.16 \mathrm{~g}, 28.0 \mathrm{mmol}, 92 \%\right.$ yield) as a colorless liquid. ${ }^{1} \mathrm{H} \mathrm{NMR}(400 \mathrm{MHz}$, $\left.\mathrm{CDCl}_{3}\right) \delta 1.08$ (m, $\left.21 \mathrm{H}, \mathrm{TIPS}\right), 0.18$ (s, 9 H, TMS). IR $v 2959$ (m), 2944 (m), 2896 (w), 2867 (m), 1464 (w), $1385(\mathrm{w}), 1250(\mathrm{~m}), 996(\mathrm{w}), 842(\mathrm{~s}), 764(\mathrm{~s}), 675(\mathrm{~m}), 660(\mathrm{~m})$. Characterization data of 10 corresponded to the literature values. ${ }^{1}$

1-Hydroxy-1,2-benziodoxol-3(1H)-one (12)

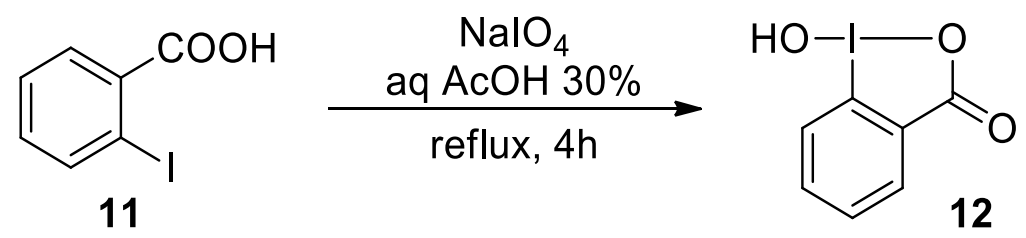

Following a reported procedure, ${ }^{2} \mathrm{NaIO}_{4}(6.7 \mathrm{~g}, 31 \mathrm{mmol} ; 1.0$ equiv) and 2-iodobenzoic acid (11) (7.4 g, $30 \mathrm{mmol}, 1.0$ equiv) were suspended in $30 \%$ (v:v) aq. $\mathrm{AcOH}(45 \mathrm{~mL})$. The mixture was vigorously stirred and refluxed for $4 \mathrm{~h}$. The reaction mixture was then diluted with cold water $(120 \mathrm{~mL})$ and allowed to cool to room temperature, protecting it from light. After $1 \mathrm{~h}$, the crude product was collected by filtration, washed on the filter with ice water $(3 \times 30 \mathrm{~mL})$ and acetone $(3 \times 30 \mathrm{~mL})$, and air-dried in the dark to give the pure product 12 (7.3 g, $19 \mathrm{mmol}, 92 \%$ yield) as a colorless solid. ${ }^{1} \mathrm{H}$ NMR $\left(400 \mathrm{MHz},\left(\mathrm{CD}_{3}\right)_{2} \mathrm{SO}\right) \delta 8.02(\mathrm{dd}, J=$ 7.7, $1.4 \mathrm{~Hz}, 1 \mathrm{H}, \operatorname{Ar} H), 7.97(\mathrm{~m}, 1 \mathrm{H}, \operatorname{Ar} H), 7.85(\mathrm{dd}, J=8.2,0.7 \mathrm{~Hz}, 1 \mathrm{H}, \operatorname{Ar} H), 7.71$ (td, $J=7.6,1.2 \mathrm{~Hz}, 1$ $\mathrm{H}, \mathrm{ArH}) .{ }^{13} \mathrm{C} \mathrm{NMR}\left(100 \mathrm{MHz},\left(\mathrm{CD}_{3}\right)_{2} \mathrm{SO}\right) \delta 167.7,134.5,131.5,131.1,130.4,126.3,120.4$. IR v $3083(\mathrm{w})$, 3060 (w), 2867 (w), 2402 (w), 1601 (m), 1585 (m), 1564 (m), 1440 (m), 1338 (s), 1302 (m), 1148 (m), 1018 (w), $834(\mathrm{~m}), 798(\mathrm{w}), 740(\mathrm{~s}), 694(\mathrm{~s}), 674(\mathrm{~m}), 649(\mathrm{~m})$. The characterization data for compounds 12 corresponded to the reported values. ${ }^{2}$

\section{1-[(Triisopropyllsilyl)ethynyl]-1,2-benziodoxol-3(1H)-one (TIPS-EBX, 1)}

(1) Helal, C J.; Magriotis, P. A; Corey, E. J. J. Am. Chem. Soc. 1996, 118, 10938.

(2) Kraszkiewicz, L.; Skulski, L. Arkivoc 2003, 6, 120. 


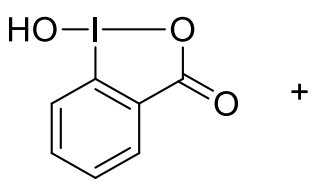

12

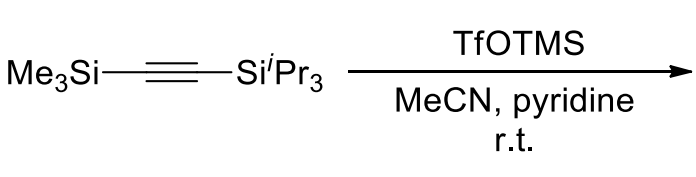

10

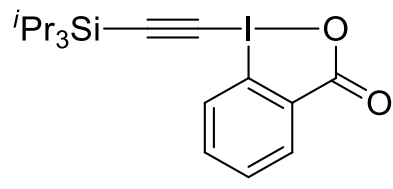

1

2-iodosylbenzoic acid (12) (21.7 g, $82.0 \mathrm{mmol}, 1.0$ equiv) was charged in oven-dried three-neck 1L flask equipped with a magnetic stirred. After 3 vacuum/nitrogen cycles, anhydrous acetonitrile $(500 \mathrm{~mL})$ was added via canula and cooled to $4{ }^{\circ} \mathrm{C}$. Trimethylsilyltriflate $(16.4 \mathrm{~mL}, 90.0 \mathrm{mmol}, 1.1$ equiv) was added dropwise via a dropping funnel over $30 \mathrm{~min}$ (no temperature increase was observed). After $15 \mathrm{~min}$, (trimethylsilyl)(triisopropylsilyl)acetylene (10) (23.0 g, $90.0 \mathrm{mmol}, 1.1$ equiv) was added via canula over 15 min (no temperature increase was observed). After $30 \mathrm{~min}$, the suspension became an orange solution. After $10 \mathrm{~min}$, pyridine $(7.0 \mathrm{~mL}, 90 \mathrm{mmol}, 1.1$ equiv) was added via syringe. After $15 \mathrm{~min}$, the reaction mixture was transferred in a one-neck $1 \mathrm{~L}$ flask and reduced under vacuum until a solid was obtained. The solid was dissolved in $\mathrm{CH}_{2} \mathrm{Cl}_{2}(200 \mathrm{~mL})$ and transferred in a 1L separatory funnel. The organic layer was added and washed with $1 \mathrm{M} \mathrm{HCl}(200 \mathrm{~mL})$ and the aqueous layer was extracted with $\mathrm{CH}_{2} \mathrm{Cl}_{2}(200 \mathrm{~mL})$. The organic layers were combined, washed with a saturated solution of $\mathrm{NaHCO}_{3}(2 \times 200 \mathrm{~mL})$, dried over $\mathrm{MgSO}_{4}$, filtered and the solvent was evaporated under reduced pressure. Recrystallization from acetonitrile ( $\mathrm{ca} 120$ $\mathrm{mL})$ afforded $1(30.1 \mathrm{~g}, 70.2 \mathrm{mmol}, 86 \%)$ as colorless cristals. Mp (Dec.) 170-176 ${ }^{\circ} \mathrm{C} .{ }^{1} \mathrm{H} \mathrm{NMR}(400 \mathrm{MHz}$, $\left.\mathrm{CDCl}_{3}\right) \delta 8.44(\mathrm{~m}, 1 \mathrm{H}, \mathrm{ArH}), 8.29(\mathrm{~m}, 1 \mathrm{H}, \mathrm{ArH}), 7.77$ (m, $\left.2 \mathrm{H}, \mathrm{ArH}\right), 1.16$ (m, $\left.21 \mathrm{H}, \mathrm{TIPS}\right) .{ }^{13} \mathrm{C}$ NMR $(100$ $\left.\mathrm{MHz}, \mathrm{CDCl}_{3}\right) \delta 166.4,134.6,132.3,131.4,131.4,126.1,115.6,114.1,64.6,18.4,11.1 . \mathrm{IR} v 2943(\mathrm{~m}), 2865$ (m), 1716 (m), 1618 (m), 1604 (s), 1584 (m), 1557 (m), 1465 (m), 1439 (w), 1349 (m), 1291 (m), 1270 (w), $1244(\mathrm{~m}), 1140(\mathrm{~m}), 1016(\mathrm{~m}), 999(\mathrm{~m}), 883(\mathrm{~m}), 833(\mathrm{~m}), 742(\mathrm{~m}), 702(\mathrm{~s}), 636(\mathrm{~m})$. Characterization data of $\mathbf{1}$ corresponded to the literature values. ${ }^{3} \mathrm{~A}$ X-ray structure is available as a separate cif file.

\section{Aniline synthesis}

Anilines 5a-d and 5n are commercially available. The other anilines were synthesized following known procedures as indicated below. The used trimethoxybenzenes were all commercially available.

\section{2-Methyl- $N$-(1-phenylethyl)aniline (5e)}<smiles>Cc1ccccc1N</smiles>

13<smiles>CC(=O)c1ccccc1</smiles>

14

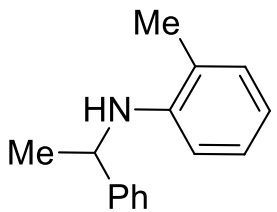

$5 e$

Following a reported procedure, ${ }^{4}$ acetophenone $(\mathbf{1 3})(0.82 \mathrm{~mL}, 7.0 \mathrm{mmol}, 1$ equiv) was added to a stirring solution of ortho-toluidine $(\mathbf{1 3})(0.75 \mathrm{~mL}, 7.0 \mathrm{mmol}, 1$ equiv) in $\mathrm{AcOH}(11.7 \mathrm{~mL})$ and $\mathrm{MeOH}(2.3 \mathrm{~mL})$. After $1 \mathrm{~h} 30, \mathrm{NaBH}_{3} \mathrm{CN}$ (480 mg, $7.70 \mathrm{mmol}, 1.1$ equiv) was added. The reaction was stirred at RT for $20 \mathrm{~h}$. The reaction was quenched with a saturated solution of $\mathrm{Na}_{2} \mathrm{CO}_{3}(10 \mathrm{~mL})$ and extracted twice with $\mathrm{CH}_{2} \mathrm{Cl}_{2}$ $(20 \mathrm{~mL})$. The organic layers were combined, dried over $\mathrm{MgSO}_{4}$, filtered and concentrated under vacuum. Purification by column chromatography (Pentane/Et ${ }_{2} \mathrm{O}$ 99/1) afforded 5e (699 mg, $3.31 \mathrm{mmol}$, 47\%) as a colorless oil. $\mathrm{R}_{\mathrm{f}}\left(\mathrm{Pentane}_{\mathrm{Et}} \mathrm{O}\right.$ 99/1): 0.2. ${ }^{1} \mathrm{H} \mathrm{NMR}\left(400 \mathrm{MHz}, \mathrm{CDCl}_{3}\right) \delta 7.34(\mathrm{~m}, 4 \mathrm{H}, \mathrm{ArH}), 7.23(\mathrm{tm}, 1 \mathrm{H}$,

(3) Zhdankin, V. V.; Kuehl, C. J.; Krasutsky, A. P.; Bolz, J. T.; Simonsen, A. J. 1996, 61, 6547.

(4) Ciana, C. L.; Phipps, R. J.; Brandt, J. R.; Meyer, F. M.; Gaunt, M. J. Angew. Chem., Int. Ed. 2011, $50,458$. 
$J=7.0 \mathrm{~Hz}, \mathrm{ArH}), 7.05(\mathrm{~d}, 1 \mathrm{H}, J=6.9 \mathrm{~Hz}, \mathrm{ArH}), 6.96(\mathrm{t}, 1 \mathrm{H}, J=7.2 \mathrm{~Hz}, \mathrm{ArH}), 6.60(\mathrm{td}, 1 \mathrm{H}, J=7.4,0.8$ $\mathrm{Hz}, \mathrm{ArH}), 6.36$ (d, $1 \mathrm{H}, J=8.1 \mathrm{~Hz}, \mathrm{ArH}), 4.54(\mathrm{~m}, 1 \mathrm{H}, \mathrm{CH}), 3.85$ (br s, $1 \mathrm{H}, J=2.3,0.5 \mathrm{~Hz}, \mathrm{NH}), 2.23$ (s, 3 $\left.\mathrm{H}, \mathrm{CH}_{3}\right), 1.56\left(\mathrm{~d}, 3 \mathrm{H}, J=6.7 \mathrm{~Hz}, \mathrm{CH}_{3}\right) .{ }^{13} \mathrm{C} \mathrm{NMR}\left(101 \mathrm{MHz}, \mathrm{CDCl}_{3}\right) \delta 145.2,145.1,129.9,128.6,127.0$, $126.8,125.7,121.5,116.8,111.0,53.3,25.3,17.6$. In accordance with reported data. ${ }^{4}$

\section{$N$-(1-phenylethyl)-[1,1'-biphenyl]-2-amine (5f)}

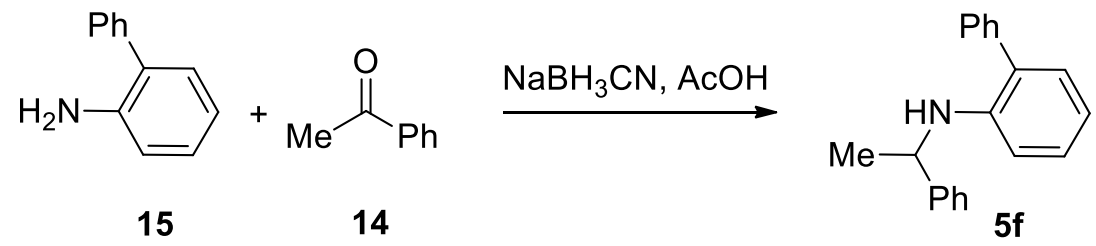

Following a reported procedure, ${ }^{4}$ acetophenone $(\mathbf{1 4})(0.82 \mathrm{~mL}, 7.0 \mathrm{mmol}, 1$ equiv) was added to a stirring solution of 2-aminodiphenyl (15) (1.25 g, $7.00 \mathrm{mmol}, 1$ equiv) in $\mathrm{AcOH}(2.3 \mathrm{~mL})$ and $\mathrm{MeOH}(11.7 \mathrm{~mL})$. After $1 \mathrm{~h}, \mathrm{NaBH}_{3} \mathrm{CN}$ (480 mg, $7.70 \mathrm{mmol}, 1.1$ equiv) was added. The reaction was stirred at RT for $20 \mathrm{~h}$. The reaction was quenched with a saturated solution of $\mathrm{Na}_{2} \mathrm{CO}_{3}$ and extracted twice with $\mathrm{CH}_{2} \mathrm{Cl}_{2}$. The organic layers were combined, dried over $\mathrm{MgSO}_{4}$, filtered and concentrated under vacuum. Purification by column chromatography (Hexane/EtOAc 99/1 to 9/1) afforded $\mathbf{5 f}$ (534 $\mathrm{mg}, 2.35 \mathrm{mmol}, 34 \%$ ) as a colorless oil. $\mathrm{R}_{\mathrm{f}}$ (Hexane/EtOAc 95/5): 0.3. ${ }^{1} \mathrm{H}$ NMR (400 MHz, $\left.\mathrm{CDCl}_{3}\right) \delta 7.50$ (m, $\left.4 \mathrm{H}, \mathrm{ArH}\right), 7.39$ (m, $\left.1 \mathrm{H}, \mathrm{ArH}\right)$, $7.32(\mathrm{~m}, 4 \mathrm{H}, \mathrm{ArH}), 7.22(\mathrm{~m}, 1 \mathrm{H}, \mathrm{ArH}), 7.06(\mathrm{~m}, 2 \mathrm{H}, \mathrm{ArH}), 6.71(\mathrm{td}, 1 \mathrm{H}, J=7.4,1.1 \mathrm{~Hz}, \operatorname{ArH}), 6.45(\mathrm{~d}, 1$ $\mathrm{H}, J=8.1 \mathrm{~Hz}, \mathrm{ArH}), 4.48(\mathrm{q}, 1 \mathrm{H}, J=6.7 \mathrm{~Hz}, \mathrm{CH}), 4.31$ (br s, $1 \mathrm{H}, \mathrm{NH}), 1.40\left(\mathrm{~d}, 3 \mathrm{H}, J=6.7 \mathrm{~Hz}, \mathrm{CH}_{3}\right) .{ }^{13} \mathrm{C}$ NMR $\left(101 \mathrm{MHz}, \mathrm{CDCl}_{3}\right) \delta 145.2,143.9,139.6,130.1,129.3,129.0,128.6,128.5,127.5,127.2,126.8$, $125.7,116.9,111.7,53.6,25.0$. Consistent with reported values. ${ }^{5}$

\section{2-Methoxy- $N$-(1-phenylethyl)aniline (5g)}
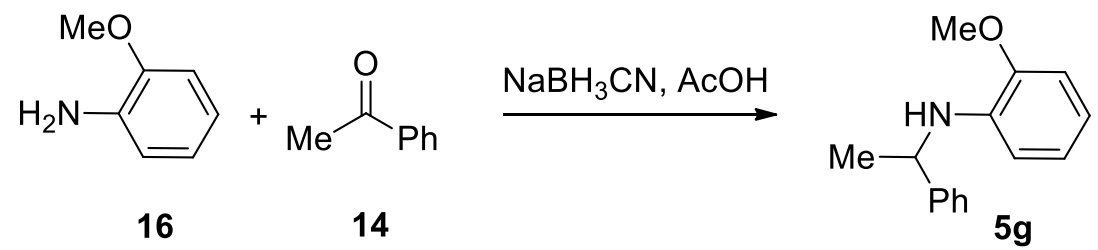

Following a reported procedure, ${ }^{4}$ acetophenone $(\mathbf{1 4})(0.59 \mathrm{~mL}, 5.0 \mathrm{mmol}, 1$ equiv $)$ was added to a stirring solution of ortho-anisidine (16) $(0.54 \mathrm{~mL}, 5.0 \mathrm{mmol}, 1$ equiv) in $\mathrm{AcOH}(1.6 \mathrm{~mL})$ and $\mathrm{MeOH}(8.4 \mathrm{~mL})$. After $45 \mathrm{~min}, \mathrm{NaBH}_{3} \mathrm{CN}$ (344 mg, $5.50 \mathrm{mmol}, 1.1$ equiv) was added. The reaction was stirred at RT for $20 \mathrm{~h}$. The reaction was quenched with saturated solution of $\mathrm{Na}_{2} \mathrm{CO}_{3}$ and extracted twice with $\mathrm{CH}_{2} \mathrm{Cl}_{2}$. The organic layers were combined, dried over $\mathrm{MgSO}_{4}$, filtered and concentrated under vacuum. Purification by column chromatography (Pentane/Et $2 \mathrm{O}$ 98/2) afforded 5g (432 mg, $1.91 \mathrm{mmol}, 38 \%$ ) as a colorless solid. $\mathrm{R}_{\mathrm{f}}$ (Pentane/Et ${ }_{2} \mathrm{O}$ 98/2): 0.25. Mp: 56-57 ${ }^{\circ} \mathrm{C} .{ }^{1} \mathrm{H} \mathrm{NMR}\left(400 \mathrm{MHz}, \mathrm{CDCl}_{3}\right) \delta 7.40$ (m, $\left.2 \mathrm{H}, \mathrm{ArH}\right), 7.34(\mathrm{t}, 2 \mathrm{H}, J$ $=7.3 \mathrm{~Hz}, \operatorname{ArH}), 7.25(\mathrm{~m}, 1 \mathrm{H}, \mathrm{ArH}), 6.80(\mathrm{~d}, 1 \mathrm{H}, J=7.8 \mathrm{~Hz}, \operatorname{ArH}), 6.73(\mathrm{~m}, 1 \mathrm{H}, \mathrm{ArH}), 6.64(\mathrm{~m}, 1 \mathrm{H}$, ArH), $6.38(\mathrm{~d}, 1 \mathrm{H}, J=7.8 \mathrm{~Hz}, \mathrm{ArH}), 4.66(\mathrm{~s}, 1 \mathrm{H}, \mathrm{NH}), 4.51(\mathrm{q}, 1 \mathrm{H}, J=6.2 \mathrm{~Hz}, \mathrm{CH}), 3.91\left(\mathrm{~s}, 3 \mathrm{H}, \mathrm{CH}_{3}\right)$, $1.58\left(\mathrm{~m}, 3 \mathrm{H}, \mathrm{CH}_{3}\right) .{ }^{13} \mathrm{C} \mathrm{NMR}\left(101 \mathrm{MHz}, \mathrm{CDCl}_{3}\right) \delta 146.5,145.4,137.2,128.5,126.7,125.8,121.1,116.3$, 111.0, 109.2, 55.4, 53.3, 25.1. IR 3426 (w), 3065 (w), 3027 (w), 2965 (w), $2954(w), 2866(w), 2834(w)$, 1603 (m), 1511 (s), 1456 (m), 1447 (m), 1373 (w), 1346 (w), 1279 (w), 1252 (s), 1223 (s), 1177 (w), 1144

(5) R. E. Meadows, S. Woodward, Tetrahedron 2008, 64, 1218. 
(m), 1110 (w), 1058 (w), 1029 (s), 910 (w), 764 (m), 735 (s), 701 (s). HRMS (ESI) calcd for $\mathrm{C}_{15} \mathrm{H}_{18} \mathrm{NO}^{+}$ $[\mathrm{M}+\mathrm{H}]^{+} 228.1383$; found 228.1380 .

\section{$N, N$-Dibenzylnaphthalen-1-amine (5h)}

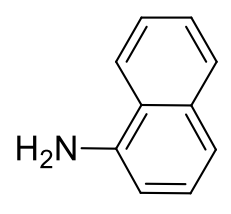

17

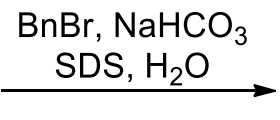

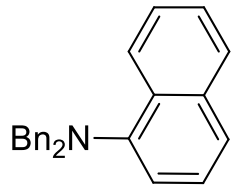

$5 \mathrm{~h}$

Following a reported procedure, ${ }^{6}$ benzyl bromide $(1.83 \mathrm{~mL}, 15.4 \mathrm{mmol}, 2.2$ equiv) was added to a stirring solution of 2-aminonaphthalene (17) (1.0 g, $7.0 \mathrm{mmol}, 1$ equiv), $\mathrm{NaHCO}_{3}$ (1.29 g, $15.4 \mathrm{mmol}, 2.2$ equiv) and sodium dodecyl sulfate ( $14 \mathrm{mg}, 0.49 \mathrm{mmol}, 0.007$ equiv) in water $(15 \mathrm{~mL})$ and stirred at $80{ }^{\circ} \mathrm{C}$ for $1 \mathrm{~h}$. The reaction was cooled and extracted twice with $\mathrm{CH}_{2} \mathrm{Cl}_{2}(20 \mathrm{~mL})$. The organic layers were dried on $\mathrm{MgSO}_{4}$ and concentrated under vacuum. The resulting oil was purified by column chromatography (pentane/Et ${ }_{2} \mathrm{O}$ 99/1) to afford $\mathbf{5 h}(1.93 \mathrm{~g}, 5.98 \mathrm{mmol}, 85 \%)$ as a colorless oil. $\mathrm{R}_{\mathrm{f}}\left(\right.$ pentane/Et $_{2} \mathrm{O}$ 99/1): 0.25. ${ }^{1} \mathrm{H}$ NMR (400 $\left.\mathrm{MHz}, \mathrm{CDCl}_{3}\right) \delta 8.62(\mathrm{~d}, 1 \mathrm{H}, J=8.2 \mathrm{~Hz}, \mathrm{ArH}), 7.91(\mathrm{~d}, 1 \mathrm{H}, J=8.0 \mathrm{~Hz}, \mathrm{ArH}), 7.51-7.64(\mathrm{~m}, 3 \mathrm{H}, \operatorname{ArH})$, 7.25-7.39 (m, $11 \mathrm{H}, \mathrm{ArH}), 7.00(\mathrm{~d}, 1 \mathrm{H}, J=7.4 \mathrm{~Hz}, \mathrm{ArH}), 4.38\left(\mathrm{~s}, 4 \mathrm{H}, \mathrm{CH}_{2}\right) .{ }^{13} \mathrm{C} \mathrm{NMR}\left(101 \mathrm{MHz}, \mathrm{CDCl}_{3}\right) \delta$ $147.4,138.2,134.9,129.7,128.5,128.4,128.2$, 126.9, 125.7, 125.5, 125.4, 123.7, 123.5, 118.4, 57.1. IR 3085 (w), $3061(w), 3028(w), 2920(w), 2841(w), 1949(w), 1812(w), 1594(w), 1575(m), 1509(w), 1495$ (m), 1454 (m), $1401(\mathrm{~m}), 1363$ (w), 1259 (w), 1223 (w), 1155 (w), 1100 (w), 1077 (w), $1029(\mathrm{w}), 1001(\mathrm{w})$, 951 (w), 909 (m), 798 (m), 775 (s), 735 (s), 698 (s). HRMS (ESI) calcd for $\mathrm{C}_{24} \mathrm{H}_{22} \mathrm{~N}^{+}[\mathrm{M}+\mathrm{H}]^{+}$324.1747; found 324.1737 .

\section{2-Methyl- $N$-(1-phenylethyl)aniline (5i)}

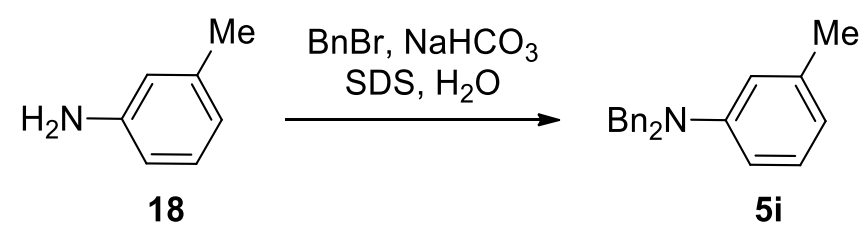

Following a reported procedure, ${ }^{6}$ benzyl bromide $(1.32 \mathrm{~mL}, 11.0 \mathrm{mmol}, 2.2$ equiv $)$ was added to a stirring solution of $m$-toluidine (18) (535 mg, $5.00 \mathrm{mmol}, 1$ equiv), $\mathrm{NaHCO}_{3}(924 \mathrm{mg}, 11.0 \mathrm{mmol}, 1$ equiv) and sodium dodecyl sulfate $\left(20 \mathrm{mg}, 0.069 \mathrm{mmol}, 0.14\right.$ equiv) in water $(20 \mathrm{~mL})$ at $80{ }^{\circ} \mathrm{C}$. After $1 \mathrm{~h}$, the reaction was cooled and extracted twice with $\mathrm{CH}_{2} \mathrm{Cl}_{2}(20 \mathrm{~mL})$. The organic layers were dried on $\mathrm{MgSO}_{4}$ and concentrated under vacuum. The resulting oil was purified by column chromatography (pentane/Et $2 \mathrm{O} 99 / 1$ ) to afford $5 \mathbf{i}(1.3 \mathrm{~g}, 4.6 \mathrm{mmol}, 92 \%)$ as a colorless solid. $\mathrm{R}_{\mathrm{f}}$ (pentane/Et $\mathrm{E}_{2} \mathrm{O}$ 99/1): 0.2. Mp: 77-78 ${ }^{\circ} \mathrm{C} .{ }^{1} \mathrm{H} \mathrm{NMR}$ $\left(400 \mathrm{MHz}, \mathrm{CDCl}_{3}\right) \delta 7.32(\mathrm{~m}, 10 \mathrm{H}, \mathrm{ArH}), 7.11(\mathrm{~m}, 1 \mathrm{H}, \mathrm{ArH}), 6.61(\mathrm{~m}, 3 \mathrm{H}, \mathrm{ArH}), 4.67\left(\mathrm{~s}, 4 \mathrm{H}, \mathrm{CH}_{2}\right), 2.29$ $\left(\mathrm{s}, 3 \mathrm{H}, \mathrm{CH}_{3}\right) .{ }^{13} \mathrm{C} \mathrm{NMR}\left(101 \mathrm{MHz}, \mathrm{CDCl}_{3}\right) \delta 149.4,138.9,138.7,129.1,128.6,126.8,126.7,117.7,113.0$, 109.7, 54.0, 21.9. IR 3085 (w), 3028 (w), 2912 (w), 2862 (w), 1949 (w), 1809 (w), 1602 (s), 1573 (m), 1495 (s), 1451 (s), 1390 (m), 1360 (m), 1328 (w), $1297(\mathrm{w}), 1249$ (m), $1188(\mathrm{~m}), 1181(\mathrm{~m}), 1075(\mathrm{w}), 1029$ (w), $991(\mathrm{~m}), 961$ (m), $911(\mathrm{w}), 838$ (w), 767 (s), 736 (s), 695 (s). HRMS (ESI) calcd for $\mathrm{C}_{21} \mathrm{H}_{22} \mathrm{~N}^{+}[\mathrm{M}+\mathrm{H}]^{+}$ 288.1747; found 288.1734.

(6) Singh, C. B.; Kavala, V.; Samal, A. K.; Patel, B. K. Eur. J. Org. Chem. 2007, 1369 


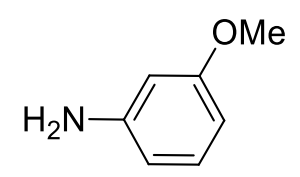

19

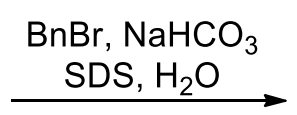

SDS, $\mathrm{H}_{2} \mathrm{O}$

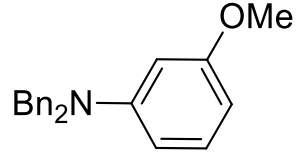

$5 \mathbf{j}$

Following a reported procedure, ${ }^{6}$ benzyl bromide $(1.32 \mathrm{~mL}, 11.0 \mathrm{mmol}, 2.2$ equiv) was added to a stirring solution of $m$-anisidine (19) $\left(559 \mu \mathrm{L}, 5.00 \mathrm{mmol}, 1\right.$ equiv), $\mathrm{NaHCO}_{3}(924 \mathrm{mg}, 11.0 \mathrm{mmol}, 1$ equiv) and sodium dodecyl sulfate (20 mg, $0.069 \mathrm{mmol}, 0.14$ equiv) in water $(20 \mathrm{~mL})$ and stirred at $80{ }^{\circ} \mathrm{C}$ for $1 \mathrm{~h}$. The reaction was cooled and extracted twice with $\mathrm{CH}_{2} \mathrm{Cl}_{2}(20 \mathrm{~mL})$. The organic layers were dried on $\mathrm{MgSO}_{4}$ and concentrated under vacuum. The resulting oil was purified by column chromatography (pentane/Et $2 \mathrm{O} 95 / 5$ ) to afford $5 \mathbf{j}(1.4 \mathrm{~g}, 4.6 \mathrm{mmol}, 92 \%)$ as a colorless oil. $\mathrm{R}_{\mathrm{f}}$ (pentane/Et $\mathrm{H}_{2} \mathrm{O}$ 95/5): 0.3. ${ }^{1} \mathrm{H} \mathrm{NMR}(400 \mathrm{MHz}$, $\left.\mathrm{CDCl}_{3}\right) \delta 7.32(\mathrm{~m}, 4 \mathrm{H}, \mathrm{ArH}), 7.25(\mathrm{~m}, 6 \mathrm{H}, \mathrm{ArH}), 7.08(\mathrm{t}, 1 \mathrm{H}, J=8.1 \mathrm{~Hz}, \mathrm{ArH}), 6.37$ (m, $\left.1 \mathrm{H}, \mathrm{ArH}\right), 6.28$ $(\mathrm{m}, 2 \mathrm{H}, \mathrm{ArH}), 4.64$ (s, $\left.4 \mathrm{H}, \mathrm{ArH}, \mathrm{CH}_{2}\right), 3.69\left(\mathrm{~m}, 3 \mathrm{H}, \mathrm{CH}_{3}\right) .{ }^{13} \mathrm{C} \mathrm{NMR}\left(101 \mathrm{MHz}, \mathrm{CDCl}_{3}\right) \delta 160.7,150.5$, 138.5, 129.8, 128.6, 126.8, 126.6, 105.6, 101.4, 99.0, 54.9, 54.2. IR 3063 (w), 3030 (w), 2907 (w), 2832 (w), 2140 (w), 1612 (s), 1576 (m), 1500 (s), 1453 (m), 1392 (w), 1361 (m), 1329 (w), 1266 (m), 1204 (s), 1168 (s), $1054(\mathrm{w}), 1043(\mathrm{w}), 989(\mathrm{w}), 962$ (m), $911(\mathrm{w}), 827$ (w), 751 (m), 735 (s), 697 (m). HRMS (ESI) calcd for $\mathrm{C}_{21} \mathrm{H}_{22} \mathrm{NO}^{+}[\mathrm{M}+\mathrm{H}]^{+}$304.1696; found 304.1690.

\section{$N, N$-Dibenzyl-3-chloroaniline (5k)}

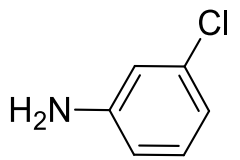

20

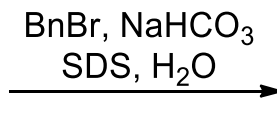

SDS, $\mathrm{H}_{2} \mathrm{O}$

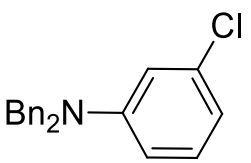

$5 \mathbf{k}$

Following a reported procedure, ${ }^{6}$ benzyl bromide $(1.32 \mathrm{~mL}, 11.0 \mathrm{mmol}, 2.2$ equiv) was added to a stirring solution of 3-chloroaniline (20) $\left(532 \mu \mathrm{L}, 5.00 \mathrm{mmol}, 1\right.$ equiv), $\mathrm{NaHCO}_{3}$ (924 mg, $11.0 \mathrm{mmol}, 1$ equiv) and sodium dodecyl sulfate (20 mg, $0.069 \mathrm{mmol}, 0.14$ equiv) in water $(20 \mathrm{~mL})$ and stirred at $80{ }^{\circ} \mathrm{C}$ for $1 \mathrm{~h}$. The reaction was cooled and extracted twice with $\mathrm{CH}_{2} \mathrm{Cl}_{2}(20 \mathrm{~mL})$. The organic layers were dried on $\mathrm{MgSO}_{4}$ and concentrated under vacuum. The resulting oil was purified by column chromatography (pentane/Et $2 \mathrm{O} 98 / 2$ ) to afford $\mathbf{5 k}(0.94 \mathrm{~g}, 3.1 \mathrm{mmol}, 76 \%)$ as a colorless oil. $\mathbf{R}_{\mathrm{f}}$ (pentane/Et $\left.{ }_{2} \mathrm{O} 98 / 2\right): 0.2 .{ }^{1} \mathrm{H} \mathrm{NMR}(400 \mathrm{MHz}$, $\left.\mathrm{CDCl}_{3}\right) \delta$ 7.38-7.16 (m, $\left.10 \mathrm{H}, \mathrm{ArH}\right), 7.06(\mathrm{t}, 1 \mathrm{H}, J=8.1 \mathrm{~Hz}, \mathrm{ArH}), 6.72(\mathrm{t}, 1 \mathrm{H}, J=2.2 \mathrm{~Hz}, \mathrm{ArH}), 6.66$ (ddd, $1 \mathrm{H}, J=7.8,1.8,0.7 \mathrm{~Hz}, \mathrm{ArH}), 6.59(\mathrm{ddd}, 1 \mathrm{H}, J=8.6,2.7,0.9 \mathrm{~Hz}, \mathrm{ArH}), 4.63\left(\mathrm{~m}, 4 \mathrm{H}, \mathrm{CH}_{2}\right) .{ }^{13} \mathrm{C} \mathrm{NMR}$ $\left(101 \mathrm{MHz} \mathrm{CDCl}_{3}\right) \delta 150.3,137.8,130.1,128.7,127.1,126.5,116.6,112.2,110.6,54.0$. One carbon not resolved. Consistent with reported values. ${ }^{6}$

\section{$N, N$-Dibenzyl-3-bromoaniline (5l)}

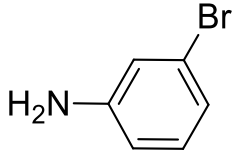

21
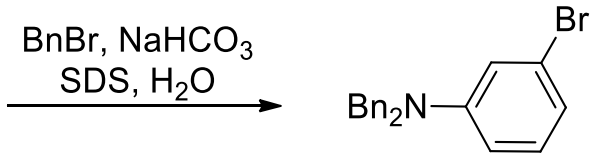

5 I

Following a reported procedure, ${ }^{6}$ benzyl bromide $(1.32 \mathrm{~mL}, 11.0 \mathrm{mmol}, 2.2$ equiv) was added to a stirring solution of 3-bromoaniline (21) (544 $\mu \mathrm{L}, 5.00 \mathrm{mmol}, 1$ equiv), $\mathrm{NaHCO}_{3}$ (924 mg, $11.0 \mathrm{mmol}, 1$ equiv) and sodium dodecyl sulfate (20 mg, $0.069 \mathrm{mmol}, 0.14$ equiv) in water $(20 \mathrm{~mL})$ and stirred at $80{ }^{\circ} \mathrm{C}$ for $2 \mathrm{~h}$. The 
reaction was cooled and extracted twice with $\mathrm{CH}_{2} \mathrm{Cl}_{2}(20 \mathrm{~mL})$. The organic layers were dried on $\mathrm{MgSO}_{4}$ and concentrated under vacuum. The resulting oil was purified by column chromatography (Hexane/EtOAc 99/1 to 9/1) and then purified by recrystallization in $\mathrm{H}_{2} \mathrm{O} / \mathrm{EtOH}$ to afford $\mathbf{5 l}$ (708 $\mathrm{mg}, 1.93 \mathrm{mmol}, 39 \%$ ) as colorless needles. $\mathrm{R}_{\mathrm{f}}$ (pentane/EtOAc 95/5): 0.5. Mp: 94-95 ${ }^{\circ} \mathrm{C} .{ }^{1} \mathrm{H}$ NMR (400 MHz, $\left.\mathrm{CDCl}_{3}\right) \delta 7.34(\mathrm{~m}, 4 \mathrm{H}$, ArH), 7.30-7.19 (m, $6 \mathrm{H}, \mathrm{ArH}), 7.00(\mathrm{~m}, 1 \mathrm{H}, J=7.9 \mathrm{~Hz}, \mathrm{ArH}), 6.89$ (t, $1 \mathrm{H}, J=1.8 \mathrm{~Hz}, \operatorname{ArH}), 6.81(\mathrm{~d}$, $1 \mathrm{H}, J=7.8 \mathrm{~Hz}, \mathrm{ArH}), 6.63(\mathrm{~d}, 1 \mathrm{H}, J=8.4 \mathrm{~Hz}, \mathrm{ArH}), 4.63\left(\mathrm{~s}, 4 \mathrm{H}, \mathrm{CH}_{2}\right) .{ }^{13} \mathrm{C} \mathrm{NMR}\left(101 \mathrm{MHz}, \mathrm{CDCl}_{3}\right) \delta$ $150.4,137.7,130.4,128.7,127.1,126.5,123.5,119.6,115.0,111.0,54.0$. Consistent with reported data. ${ }^{7}$

\section{$N, N$-Dibenzyl-4-methylaniline (23)}

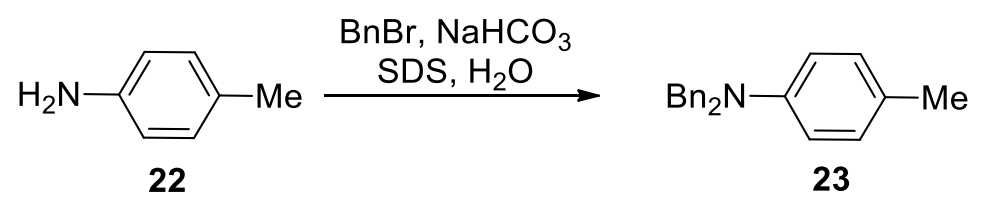

Following a reported procedure, ${ }^{6}$ benzyl bromide $(2.62 \mathrm{~mL}, 22.0 \mathrm{mmol}, 2.2$ equiv) was added to a stirring solution of $p$-toluidine (22) (1.07 g, $10.0 \mathrm{mmol}, 1$ equiv), $\mathrm{NaHCO}_{3}$ (1.85 g, $22.0 \mathrm{mmol}, 1$ equiv) and sodium dodecyl sulfate $\left(20 \mathrm{mg}, 0.069 \mathrm{mmol}, 0.14\right.$ equiv) in water $(20 \mathrm{~mL})$ and stirred at $80{ }^{\circ} \mathrm{C}$ for $1 \mathrm{~h}$. The reaction was cooled and extracted twice with $\mathrm{CH}_{2} \mathrm{Cl}_{2}(20 \mathrm{~mL})$. The organic layers were dried on $\mathrm{MgSO}_{4}$ and concentrated under vacuum. The resulting oil was purified by column chromatography (pentane/Et ${ }_{2} \mathrm{O}$ 99/1 to 98/2) to afford 23 (2.04 g, $7.10 \mathrm{mmol}, 71 \%)$ as a colorless solid. $\mathrm{R}_{\mathrm{f}}$ (pentane/Et $\mathrm{t}_{2} \mathrm{O}$ 99/1): 0.15. Mp: 56$57^{\circ} \mathrm{C} .{ }^{1} \mathrm{H}$ NMR $\left(400 \mathrm{MHz}, \mathrm{CDCl}_{3}\right) \delta 7.47(\mathrm{~m}, 4 \mathrm{H}, \mathrm{ArH}), 7.38(\mathrm{~m}, 6 \mathrm{H}, \mathrm{ArH}), 7.13(\mathrm{~d}, 2 \mathrm{H}, J=8.7 \mathrm{~Hz}$, $\operatorname{ArH}), 6.81(\mathrm{~d}, 2 \mathrm{H}, J=8.6 \mathrm{~Hz}, \mathrm{ArH}), 4.77\left(\mathrm{~s}, 4 \mathrm{H}, \mathrm{CH}_{2}\right), 2.38\left(\mathrm{~s}, 3 \mathrm{H}, \mathrm{CH}_{3}\right) .{ }^{13} \mathrm{C} \mathrm{NMR}\left(101 \mathrm{MHz}, \mathrm{CDCl}_{3}\right) \delta$ 147.0, 138.8, 129.7, 128.6, 126.8, 126.7, 125.8, 112.6, 54.4, 20.2. Consistent with reported data. ${ }^{6}$

\section{Alkynylation}

\section{$N, N$-Dibenzyl-4-((triisopropylsilyl)ethynyl)aniline (6a)}

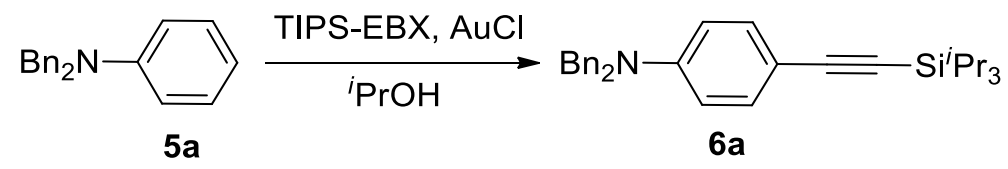

$\mathrm{AuCl}$ (4.6 mg, $0.020 \mathrm{mmol}, 0.05$ equiv) was added to a stirring solution of $N, N$-dibenzylaniline (5a) (109 mg, 0.400 mmol, 1 equiv) and TIPS-EBX (1) $\left(240 \mathrm{mg}, 0.560 \mathrm{mmol}, 1.4\right.$ equiv) in ${ }^{i} \mathrm{PrOH}^{8}(8 \mathrm{~mL})$ under air. The reaction was stirred at RT for $24 \mathrm{~h}$. EtOAc $(20 \mathrm{~mL})$ was added to the reaction mixture. The organic mixture was then washed with $0.1 \mathrm{M} \mathrm{NaOH}(20 \mathrm{~mL})$, a saturated solution of $\mathrm{NaHCO}_{3}(20 \mathrm{~mL})$ and brine $(20$ $\mathrm{mL}$ ), dried over $\mathrm{MgSO}_{4}$ and concentrated in vacuum. The resulting oil was purified by column chromatography (pentane/ $\left.\mathrm{CH}_{2} \mathrm{Cl}_{2} 9 / 1\right)$ to afford $\mathbf{6 a}(126 \mathrm{mg}, 0.278 \mathrm{mmol}, 70 \%)$ as a colorless oil and a mixture of 5a and 6a $(20 \mathrm{mg}, 30 \mathrm{w} \%$ product and $70 \mathrm{w} \% \mathrm{~N}, \mathrm{~N}$-dibenzylaniline, $3 \%$ product and $13 \%$ recovered starting material) as a colorless oil. Total yield $=73 \%$. Yield based on recovered starting material $=84 \% . \mathrm{R}_{\mathrm{f}}$ (pentane $/ \mathrm{CH}_{2} \mathrm{Cl}_{2}$ 9/1) 0.15. ${ }^{1} \mathrm{H}$ NMR (400 MHz, $\left.\mathrm{C}_{6} \mathrm{D}_{6}\right) \delta 7.37$ (m, $\left.2 \mathrm{H}, \mathrm{ArH}\right), 7.18-7.01(\mathrm{~m}, 6 \mathrm{H}$, ArH), 6.95 (m, 4 H, ArH), 6.42 (m, $2 \mathrm{H}, \mathrm{ArH}), 4.15\left(\mathrm{~s}, 4 \mathrm{H}, \mathrm{CH}_{2}\right), 1.21$ (m, $\left.21 \mathrm{H}, \mathrm{TIPS}\right) .{ }^{13} \mathrm{C}$ NMR (101 $\left.\mathrm{MHz}, \mathrm{C}_{6} \mathrm{D}_{6}\right) \delta 149.3,138.4,133.9,128.9,127.3,126.9,112.4,111.8,109.6,87.6,53.9,19.1,11.9$. IR 3051 (w), $2943(\mathrm{w}), 2865(\mathrm{w}), 2143(\mathrm{w}), 1606(\mathrm{~m}), 1516(\mathrm{~m}), 1495(\mathrm{w}), 1454(\mathrm{w}), 1398(\mathrm{w}), 1360(\mathrm{w}), 1266(\mathrm{~m})$,

(7) Feng, C.; Liu, Y.; Peng, S.; Shuai, Q.; Deng, G.; Li, C.-J. Org. Lett. 2010, 12, 4888.

(8) Commercial isopropanol was used without drying procedure. 
1241 (w), 1188 (w), 1075 (w), 997 (w), 955 (w), 884 (w), 841 (w), 818 (w), 737 (s), 698 (m), 677 (m). HRMS (ESI) calcd for $\mathrm{C}_{31} \mathrm{H}_{40} \mathrm{NSi}^{+}[\mathrm{M}+\mathrm{H}]^{+} 454.2925$; found 454.2913.

\section{$N, N$-Dibutyl-4-((triisopropylsilyl)ethynyl)aniline (6b)}

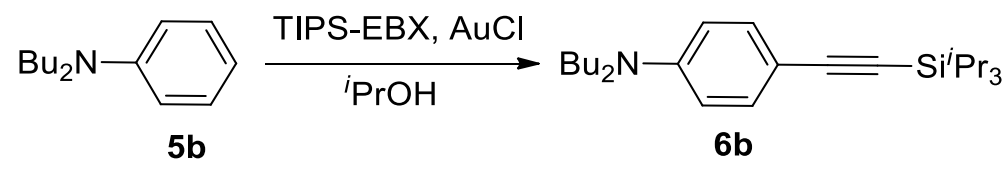

$\mathrm{AuCl}$ (4.6 mg, $0.020 \mathrm{mmol}, 0.05$ equiv) was added to a stirring solution of $N, N$-dibutylaniline (5b) (91 $\mathrm{mg}$, $0.40 \mathrm{mmol}, 1$ equiv) and TIPS-EBX (1) $\left(240 \mathrm{mg}, 0.560 \mathrm{mmol}, 1.4\right.$ equiv) in ${ }^{i} \mathrm{PrOH}^{8}(2 \mathrm{~mL})$ under air. The reaction was stirred at RT for $20 \mathrm{~h}$. EtOAc $(20 \mathrm{~mL})$ was added to the reaction mixture. The organic mixture was then washed with $0.1 \mathrm{M} \mathrm{NaOH}(20 \mathrm{~mL})$, a saturated solution of $\mathrm{NaHCO}_{3}(20 \mathrm{~mL})$ and brine $(20 \mathrm{~mL})$, dried over $\mathrm{MgSO}_{4}$ and concentrated in vacuum. The resulting oil was purified by column chromatography (pentane) to afford $\mathbf{6 b}(97 \mathrm{mg}, 0.25 \mathrm{mmol}, 63 \%)$ as a colorless oil. $\mathrm{R}_{\mathrm{f}}$ (pentane) 0.3. ${ }^{1} \mathrm{H} \mathrm{NMR} \mathrm{(400} \mathrm{MHz,}$ $\left.\mathrm{CDCl}_{3}\right) \delta 7.32(\mathrm{~m}, 2 \mathrm{H}, \mathrm{ArH}), 6.53(\mathrm{~m}, 2 \mathrm{H}, \mathrm{ArH}), 3.27\left(\mathrm{~m}, 4 \mathrm{H}, \mathrm{CH}_{2}\right), 1.56\left(\mathrm{~m}, 4 \mathrm{H}, \mathrm{CH}_{2}\right), 1.35(\mathrm{~m}, 4 \mathrm{H}$, $\left.\mathrm{CH}_{2}\right), 1.13(\mathrm{~m}, 21 \mathrm{H}, \mathrm{TIPS}), 0.95\left(\mathrm{t}, 6 \mathrm{H}, J=7.3 \mathrm{~Hz}, \mathrm{CH}_{3}\right) .{ }^{13} \mathrm{C} \mathrm{NMR}\left(101 \mathrm{MHz}, \mathrm{CDCl}_{3}\right) \delta 147.9,133.3$, 111.1, 109.2, 108.6, 86.8, 50.7, 29.4, 20.3, 18.7, 14.0, 11.5. IR 2958 (m), 2942 (m), 2864 (s), 2142 (m), 1607 (s), 1514 (s), 1463 (m), 1401 (w), 1368 (m), 1288 (w), 1222 (w), 1187 (m), 1150 (w), 1111 (w), 1069 (w), $1017(\mathrm{w}), 996(\mathrm{w}), 923$ (w), $883(\mathrm{~m}), 839$ (m), 814 (m), 734 (m), 676 (m), 634 (s). HRMS (ESI) calcd for $\mathrm{C}_{25} \mathrm{H}_{44} \mathrm{NSi}^{+}[\mathrm{M}+\mathrm{H}]^{+}$386.3238; found 386.3225 .

\section{$N, N$-Diethyl-4-((triisopropylsilyl)ethynyl)aniline (6c)}

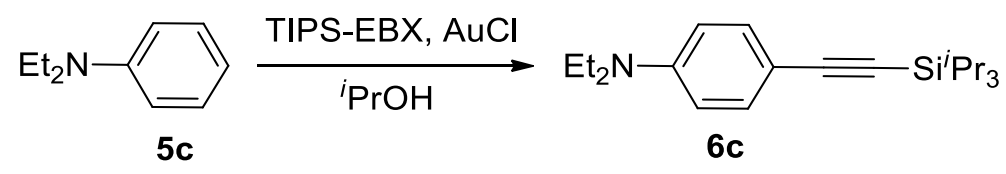

$\mathrm{AuCl}$ (4.6 mg, $0.020 \mathrm{mmol}, 0.05$ equiv) was added to a stirring solution of $N, N$-diethylaniline (5c) $(64 \mu \mathrm{L}$, $0.40 \mathrm{mmol}, 1$ equiv) and TIPS-EBX (1) $\left(240 \mathrm{mg}, 0.560 \mathrm{mmol}, 1.4\right.$ equiv) in ${ }^{i} \operatorname{PrOH}^{8}(8 \mathrm{~mL})$ under air. The reaction was stirred at $\mathrm{RT}$ for $36 \mathrm{~h}$. EtOAc $(20 \mathrm{~mL})$ was added to the reaction mixture. The organic mixture was then washed with $0.1 \mathrm{M} \mathrm{NaOH}(20 \mathrm{~mL})$, a saturated solution of $\mathrm{NaHCO}_{3}(20 \mathrm{~mL})$ and brine $(20 \mathrm{~mL})$, dried over $\mathrm{MgSO}_{4}$ and concentrated in vacuum. The resulting oil was purified by column chromatography (pentane $/ \mathrm{CH}_{2} \mathrm{Cl}_{2}$ 95/5) to afford $\mathbf{6 c}\left(58 \mathrm{mg}, 0.18 \mathrm{mmol}, 44 \%\right.$ ) as a yellow oil. $\mathrm{R}_{\mathrm{f}}\left(\right.$ pentane/ $\mathrm{CH}_{2} \mathrm{Cl}_{2}$ 9/1) 0.2. ${ }^{1} \mathrm{H}$ NMR $\left(400 \mathrm{MHz}, \mathrm{CDCl}_{3}\right) \delta 7.33(\mathrm{~m}, 2 \mathrm{H}, \mathrm{ArH}), 6.56(\mathrm{~m}, 2 \mathrm{H}, \mathrm{ArH}), 3.36\left(\mathrm{q}, 4 \mathrm{H}, J=7.1 \mathrm{~Hz}, \mathrm{CH}_{2}\right), 1.19$ $1.10\left(\mathrm{~m}, 27 \mathrm{H}, \mathrm{CH}_{3}+\mathrm{TIPS}\right) .{ }^{13} \mathrm{C} \mathrm{NMR}\left(101 \mathrm{MHz}, \mathrm{CDCl}_{3}\right) \delta 147.4,133.3,111.0,109.4,108.6,86.8,44.3$, 18.7, 12.5, 11.4. IR 2962 (m), $2941(\mathrm{~m}), 2917(\mathrm{w}), 2864(\mathrm{~m}), 2146(\mathrm{~m}), 1607$ (s), $1517(\mathrm{~s}), 1464(\mathrm{w}), 1403$ (w), $1356(\mathrm{~m}), 1270(\mathrm{~m}), 1197(\mathrm{w}), 1156(\mathrm{w}), 1076(\mathrm{w}), 997(\mathrm{w}), 920(\mathrm{w}), 883(\mathrm{~m}), 839(\mathrm{~m}), 817(\mathrm{~m}), 789$ (w), $710(\mathrm{~m}), 675$ (m). HRMS (ESI) calcd for $\mathrm{C}_{21} \mathrm{H}_{36} \mathrm{NSi}^{+}[\mathrm{M}+\mathrm{H}]^{+}$330.2612; found 330.2604.

\section{$N, N$-Dimethyl-4-((triisopropylsilyl)ethynyl)aniline (6d) and $N, N$-dimethyl-2,4- bis((triisopropylsilyl)ethynyl)aniline (24)}

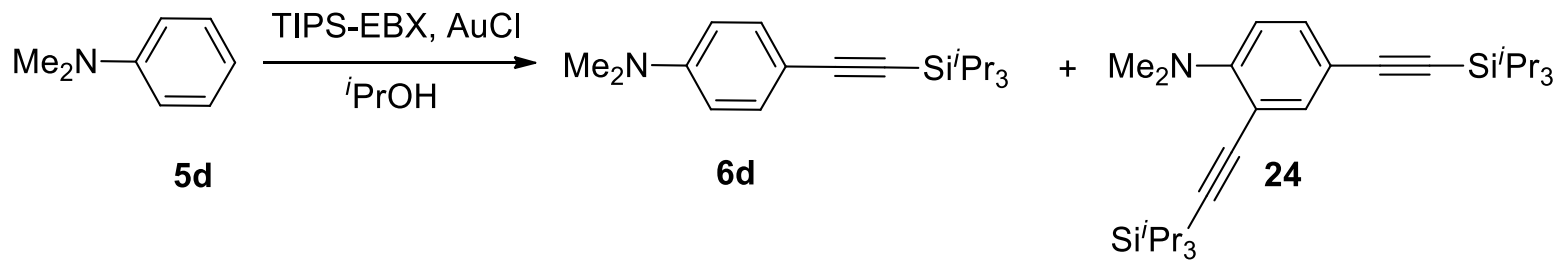


$\mathrm{AuCl}$ (4.6 mg, $0.020 \mathrm{mmol}, 0.05$ equiv) was added to a stirring solution of $N, N$-dimethylaniline (5d) $(51 \mu \mathrm{L}$, $0.40 \mathrm{mmol}, 1$ equiv) and TIPS-EBX (1) $\left(240 \mathrm{mg}, 0.560 \mathrm{mmol}, 1.4\right.$ equiv) in ${ }^{i} \operatorname{PrOH}^{8}(2 \mathrm{~mL})$ under air. The reaction was stirred at $60^{\circ} \mathrm{C}$ for $20 \mathrm{~h}$. EtOAc $(20 \mathrm{~mL})$ was added to the reaction mixture. The organic mixture was then washed with $0.1 \mathrm{M} \mathrm{NaOH}(20 \mathrm{~mL})$, a saturated solution of $\mathrm{NaHCO}_{3}(20 \mathrm{~mL})$ and brine $(20$ $\mathrm{mL}$ ), dried over $\mathrm{MgSO}_{4}$ and concentrated in vacuum. The resulting oil was purified by column chromatography (pentane/ $\mathrm{CH}_{2} \mathrm{Cl}_{2}$ 95/5) to afford $24(16 \mathrm{mg}, 0.037 \mathrm{mmol}, 8 \%)$ as a colorless oil and $\mathbf{6 d}(55$ $\mathrm{mg}, 0.18 \mathrm{mmol}, 46 \%$ ) as a yellow oil. 24: $\mathrm{R}_{\mathrm{f}}$ (pentane $/ \mathrm{CH}_{2} \mathrm{Cl}_{2}$ 95/5) 0.3. ${ }^{1} \mathrm{H} \mathrm{NMR}\left(400 \mathrm{MHz}, \mathrm{CDCl}_{3}\right) \delta 7.50$ $(\mathrm{d}, 1 \mathrm{H}, J=2.1 \mathrm{~Hz}, \mathrm{ArH}), 7.30(\mathrm{dd}, 1 \mathrm{H}, J=8.6,2.1 \mathrm{~Hz}, \mathrm{ArH}), 6.73(\mathrm{~d}, 1 \mathrm{H}, J=8.6 \mathrm{~Hz}, \mathrm{ArH}), 2.99$ (s, $6 \mathrm{H}$, $\mathrm{Me}), 1.13$ (m, $42 \mathrm{H}$, TIPS). ${ }^{13} \mathrm{C}$ NMR $\left(101 \mathrm{MHz}, \mathrm{CDCl}_{3}\right) \delta 154.5,138.8,133.0,116.3,114.4,114.1,106.8$, 105.7, 96.4, 88.8, 43.1, 43.1, 18.7, 18.6, 11.4. IR 2943 (m), $2876(\mathrm{w}), 2865(\mathrm{~m}), 2144(\mathrm{~m}), 1597(\mathrm{w}), 1498$ (m), 1462 (m), 1387 (w), 1335 (w), $1271(\mathrm{w}), 1245$ (w), 1178 (w), 1138 (w), $1112(\mathrm{w}), 1072(\mathrm{w}), 1015(\mathrm{w})$, $997(\mathrm{w}), 940(\mathrm{~m}), 883(\mathrm{~m}), 821(\mathrm{w}), 779(\mathrm{w}), 723(\mathrm{~m}), 673$ (s). HRMS (ESI) calcd for $\mathrm{C}_{30} \mathrm{H}_{52} \mathrm{NSi}_{2}{ }^{+}[\mathrm{M}+\mathrm{H}]^{+}$ 482.3633; found 482.3628. 6d: Rf (pentane/ $\mathrm{CH}_{2} \mathrm{Cl}_{2}$ 95/5) 0.2. ${ }^{1} \mathrm{H} \mathrm{NMR}\left(400 \mathrm{MHz}, \mathrm{CDCl}_{3}\right) \delta 7.36(\mathrm{~m}, 2 \mathrm{H}$, $\mathrm{ArH}), 6.61(\mathrm{~d}, 2 \mathrm{H}, J=8.9 \mathrm{~Hz}, \mathrm{ArH}), 2.97$ (s, $6 \mathrm{H}, \mathrm{Me}), 1.14$ (m, $21 \mathrm{H}, \mathrm{TIPS}) .{ }^{13} \mathrm{C} \mathrm{NMR}\left(101 \mathrm{MHz}, \mathrm{CDCl}_{3}\right)$ $\delta 150.1,133.1,111.6,110.6,108.3,87.2,40.2,18.7,11.4$. IR $2941(\mathrm{~m}), 2890(\mathrm{~m}), 2864(\mathrm{~m}), 2150(\mathrm{~m}), 1713$ (w), 1608 (s), 1519 (s), 1462 (m), 1360 (m), $1238(\mathrm{~m}), 1186(\mathrm{w}), 1168(\mathrm{w}), 1070(\mathrm{w}), 996(\mathrm{w}), 949(\mathrm{~m}), 909$ (m), 883 (m), 839 (s), 817 (s), 724 (s), 676 (s). HRMS (ESI) calcd for $\mathrm{C}_{19} \mathrm{H}_{32} \mathrm{NSi}^{+}[\mathrm{M}+\mathrm{H}]^{+}$302.2299; found 302.2297.

2-Methyl- $N$-(1-phenylethyl)-4-((triisopropylsilyl)ethynyl)aniline (6e)

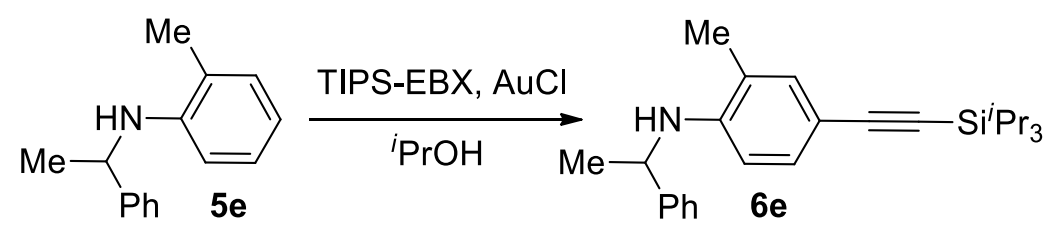

$\mathrm{AuCl}$ (4.6 mg, $0.020 \mathrm{mmol}, 0.05$ equiv) was added to a stirring solution of $\mathbf{5 e}$ ( $85 \mathrm{mg}, 0.40 \mathrm{mmol}, 1$ equiv) and TIPS-EBX (1) (240 mg, $0.560 \mathrm{mmol}, 1.4$ equiv) in ${ }^{i} \mathrm{PrOH}^{8}(2 \mathrm{~mL})$ under air. The reaction was stirred at $60^{\circ} \mathrm{C}$ for $20 \mathrm{~h}$. EtOAc $(20 \mathrm{~mL})$ was added to the reaction mixture. The organic mixture was then washed with $0.1 \mathrm{M} \mathrm{NaOH}(20 \mathrm{~mL})$, a saturated solution of $\mathrm{NaHCO}_{3}(20 \mathrm{~mL})$ and brine $(20 \mathrm{~mL})$, dried over $\mathrm{MgSO}_{4}$ and concentrated in vacuum. The resulting oil was purified by column chromatography (pentane/ $\mathrm{CH}_{2} \mathrm{Cl}_{2}$ 9/1) to afford $6 \mathrm{e}(100 \mathrm{mg}, 0.255 \mathrm{mmol}, 64 \%)$ as a yellow oil. $\mathrm{R}_{\mathrm{f}}$ (pentane/ $\mathrm{CH}_{2} \mathrm{Cl}_{2}$ 9/1): 0.15. ${ }^{1} \mathrm{H} \mathrm{NMR}(400$ $\left.\mathrm{MHz}, \mathrm{CDCl}_{3}\right) \delta 7.35(\mathrm{~m}, 4 \mathrm{H}, \mathrm{ArH}), 7.27(\mathrm{~m}, 2 \mathrm{H}, \mathrm{ArH}), 7.15(\mathrm{dd}, 1 \mathrm{H}, J=8.3,1.6 \mathrm{~Hz}, \mathrm{ArH}), 6.30(\mathrm{~d}, 1 \mathrm{H}, J$ $=8.4 \mathrm{~Hz}, \mathrm{ArH}), 4.60(\mathrm{~m}, 1 \mathrm{H}, \mathrm{CH}), 4.07(\mathrm{br} \mathrm{d}, 1 \mathrm{H}, J=4.6 \mathrm{~Hz}, \mathrm{NH}), 2.24(\mathrm{~s}, 3 \mathrm{H}, \mathrm{Me}), 1.60(\mathrm{~d}, 3 \mathrm{H}, J=6.7$ $\mathrm{Hz}, \mathrm{Me}), 1.18(\mathrm{~m}, 21 \mathrm{H}, \mathrm{TIPS}) .{ }^{13} \mathrm{C} \mathrm{NMR}\left(101 \mathrm{MHz}, \mathrm{CDCl}_{3}\right) \delta 145.2,144.7,133.7,131.4,128.8,127.1$, 125.7, 121.3, 111.2, 110.7, 108.5, 86.9, 53.1, 25.1, 18.8, 17.4, 11.5. IR 3442 (w), 2941 (m), 2864 (m), 2246 (w), $2144(\mathrm{~m}), 1608$ (m), $1574(\mathrm{w}), 1509$ (s), $1454(\mathrm{w}), 1375(\mathrm{w}), 1321(\mathrm{~m}), 1270(\mathrm{w}), 1235(\mathrm{w}), 1149(\mathrm{w})$, 1068 (w), 1016 (w), 998 (m), 992 (w), 909 (s), 884 (m), 812 (m), 752 (s), 734 (s), 701 (s), 676 (s). HRMS (ESI) calcd for $\mathrm{C}_{26} \mathrm{H}_{38} \mathrm{NSi}^{+}[\mathrm{M}+\mathrm{H}]^{+}$392.2768; found 392.2765.

\section{$N$-(1-phenylethyl)-5-((triisopropylsilyl)ethynyl)-[1,1'-biphenyl]-2-amine (6f)}

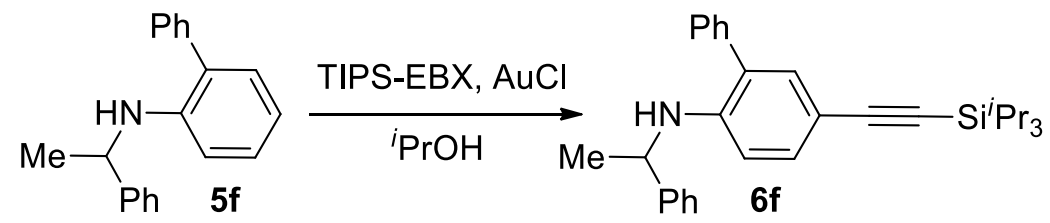


$\mathrm{AuCl}$ (4.6 mg, $0.020 \mathrm{mmol}, 0.05$ equiv) was added to a stirring solution of $\mathbf{5 f}$ (109 $\mathrm{mg}, 0.400 \mathrm{mmol}, 1$ equiv) and TIPS-EBX (1) (240 mg, $0.560 \mathrm{mmol}, 1.4$ equiv) in ${ }^{i} \mathrm{PrOH}^{8}(2 \mathrm{~mL})$ under air. The reaction was stirred at $60{ }^{\circ} \mathrm{C}$ for $20 \mathrm{~h}$. EtOAc $(20 \mathrm{~mL})$ was added to the reaction mixture. The organic mixture was then washed with $0.1 \mathrm{M} \mathrm{NaOH}(20 \mathrm{~mL})$, a saturated solution of $\mathrm{NaHCO}_{3}(20 \mathrm{~mL})$ and brine $(20 \mathrm{~mL})$, dried over $\mathrm{MgSO}_{4}$ and concentrated in vacuum. The resulting oil was purified by column chromatography (pentane/ $\mathrm{CH}_{2} \mathrm{Cl}_{2}$ 9/5) to afford $6 \mathbf{f}(117 \mathrm{mg}, 0.258 \mathrm{mmol}, 65 \%)$ as a yellow oil. $\mathrm{R}_{\mathrm{f}}$ (pentane/ $\mathrm{CH}_{2} \mathrm{Cl}_{2}$ 9/5): 0.2. ${ }^{1} \mathrm{H}$ NMR (400 $\left.\mathrm{MHz}, \mathrm{CDCl}_{3}\right) \delta$ 7.54-7.37 (m, $\left.5 \mathrm{H}, \mathrm{ArH}\right), 7.35-7.17$ (m, $\left.7 \mathrm{H}, \mathrm{ArH}\right), 6.34$ (d, $\left.1 \mathrm{H}, J=8.4 \mathrm{~Hz}, \mathrm{ArH}\right), 4.54-4.43$ $(\mathrm{m}, 2 \mathrm{H}, \mathrm{CH}+\mathrm{NH}), 1.40\left(\mathrm{~d}, 3 \mathrm{H}, J=6.6 \mathrm{~Hz}, \mathrm{CH}_{3}\right), 1.08(\mathrm{~m}, 21 \mathrm{H}, \mathrm{TIPS}) .{ }^{13} \mathrm{C} \mathrm{NMR}\left(101 \mathrm{MHz}, \mathrm{CDCl}_{3}\right) \delta$ 144.5, 143.9, 141.5, 138.6, 133.8, 132.6, 129.3, 129.1, 128.7, 127.6, 127.3, 127.0, 125.6, 111.4, 108.0, 87.4, 53.2, 24.9, 18.7, 11.4. IR 2952 (w), 2859 (w), $2255(w), 2146(w), 1722(\mathrm{~s}), 1607(w), 1511(w), 1455(w)$, 1430 (w), 1349 (w), 1256 (m), 1201 (w), 1140 (w), $1121(\mathrm{w}), 1072$ (m), $1013(\mathrm{w}), 994(\mathrm{w}), 973(\mathrm{w}), 928$ (w), 908 (s), 873 (w), 813 (w). HRMS (ESI) calcd for $\mathrm{C}_{31} \mathrm{H}_{40} \mathrm{NSi}^{+}[\mathrm{M}+\mathrm{H}]^{+}$454.2925; found 454.2922.

\section{2-Methoxy- $N$-(1-phenylethyl)-4-((triisopropylsilyl)ethynyl)aniline (6g)}

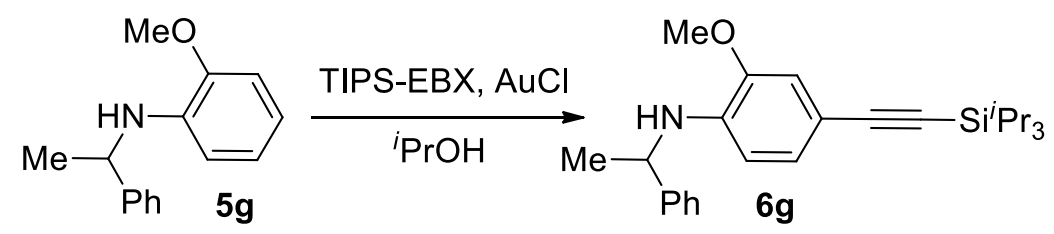

$\mathrm{AuCl}$ (4.6 mg, $0.020 \mathrm{mmol}, 0.05$ equiv) was added to a stirring solution of $\mathbf{5 g}$ (91 $\mathrm{mg}, 0.40 \mathrm{mmol}, 1$ equiv) and TIPS-EBX (1) (240 mg, $0.560 \mathrm{mmol}, 1.4$ equiv) in ${ }^{i} \mathrm{PrOH}^{8}(2 \mathrm{~mL})$ under air. The reaction was stirred at $60{ }^{\circ} \mathrm{C}$ for $24 \mathrm{~h}$. EtOAc $(20 \mathrm{~mL})$ was added to the reaction mixture. The organic mixture was then washed with $0.1 \mathrm{M} \mathrm{NaOH}(20 \mathrm{~mL})$, a saturated solution of $\mathrm{NaHCO}_{3}(20 \mathrm{~mL})$ and brine $(20 \mathrm{~mL})$, dried over $\mathrm{MgSO}_{4}$ and concentrated in vacuum. The resulting oil was purified by column chromatography (pentane/ $\mathrm{CH}_{2} \mathrm{Cl}_{2}$ 98/2) to afford $\mathbf{6 g}(62 \mathrm{mg}, 0.15 \mathrm{mmol}, 38 \%)$ as a yellow oil and a mixture of $\mathbf{6 g}$ and $\mathbf{5 g}$ (35 $\mathrm{mg}, 17 \mathrm{w} \%$ of product $(0.015 \mathrm{mmol})$ and $83 \mathrm{w} \%$ of $\mathbf{5 g}(0.13 \mathrm{mmol}), 4 \%$ product and $32 \%$ starting material recovered) as well as some pure $\mathbf{5 g}(7 \mathrm{mg}, 0.030 \mathrm{mmol}, 7 \%)$. Total yield: $42 \%$. Yield brsm $=70 \%$. $\mathrm{R}_{\mathrm{f}}\left(\right.$ pentane $/ \mathrm{CH}_{2} \mathrm{Cl}_{2}$ 98/2): 0.15. ${ }^{1} \mathrm{H}$ NMR (400 MHz, $\left.\mathrm{CDCl}_{3}\right) \delta$ 7.35-7.28 (m, $\left.4 \mathrm{H}, \mathrm{ArH}\right), 7.23$ (m, $\left.1 \mathrm{H}, \mathrm{ArH}\right), 6.89(\mathrm{~d}, 1 \mathrm{H}, J=$ $8.2 \mathrm{~Hz}, \operatorname{ArH}), 6.84$ (s, $1 \mathrm{H}, \mathrm{ArH}), 6.22(\mathrm{~d}, 1 \mathrm{H}, J=8.0 \mathrm{~Hz}, \operatorname{ArH}), 4.82$ (br s, $1 \mathrm{H}, \mathrm{NH}), 4.51$ (q, $1 \mathrm{H}, J=6.7$ $\mathrm{Hz}, \mathrm{CH}), 3.90$ (s, $3 \mathrm{H}, \mathrm{OMe}), 1.56\left(\mathrm{~d}, 3 \mathrm{H}, J=6.7 \mathrm{~Hz}, \mathrm{CH}_{3}\right), 1.12$ (m, 21H, TIPS). ${ }^{13} \mathrm{C}$ NMR $(101 \mathrm{MHz}$, $\left.\mathrm{CDCl}_{3}\right) \delta 145.7,144.8,137.6,128.6,126.9,126.2,125.7,112.4,110.5,110.3,108.5,86.8,55.5,52.9,24.9$, 18.7, 11.4. IR $3435(\mathrm{w}), 3062(\mathrm{w}), 2941(\mathrm{~m}), 2864(\mathrm{~m}), 2144(\mathrm{~m}), 1605(\mathrm{~m}), 1542(\mathrm{w}), 1520(\mathrm{~s}), 1462(\mathrm{~m})$, 1417 (w), 1353 (m), $1264(\mathrm{~m}), 1236(\mathrm{~m}), 1195(\mathrm{w}), 1146(\mathrm{~m}), 1120(\mathrm{w}), 1073(\mathrm{w}), 1038$ (m), $996(\mathrm{w}), 938$ $(\mathrm{m}), \quad 883 \quad(\mathrm{~m}), \quad 854 \quad(\mathrm{w}), \quad 805 \quad(\mathrm{~m}), \quad 752 \quad(\mathrm{~m}), 701 \quad(\mathrm{~m}), 676 \quad(\mathrm{~s})$. HRMS (ESI) calcd for $\mathrm{C}_{26} \mathrm{H}_{38} \mathrm{NOSi}^{+}[\mathrm{M}+\mathrm{H}]^{+}$408.2717; found 408.2720.

\section{$N, N$-Dibenzyl-4-((triisopropylsilyl)ethynyl)naphthalen-1-amine (6h)}

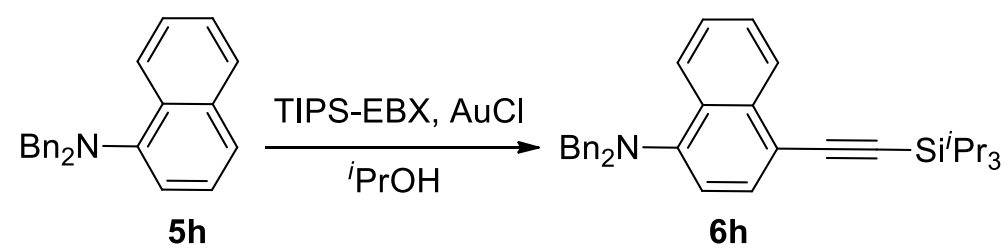

$\mathrm{AuCl}$ (4.6 mg, $0.020 \mathrm{mmol}, 0.05$ equiv) was added to a stirring solution of $\mathbf{5 h}$ (129 $\mathrm{mg}, 0.400 \mathrm{mmol}, 1$ equiv) and TIPS-EBX (1) (240 mg, $0.560 \mathrm{mmol}, 1.4$ equiv) in ${ }^{i} \mathrm{PrOH}^{8}$ (2 mL) under air. The reaction was stirred at $60{ }^{\circ} \mathrm{C}$ for $24 \mathrm{~h}$. EtOAc $(20 \mathrm{~mL})$ was added to the reaction mixture. The organic mixture was then washed with $0.1 \mathrm{M} \mathrm{NaOH}(20 \mathrm{~mL})$, a saturated solution of $\mathrm{NaHCO}_{3}(20 \mathrm{~mL})$ and brine $(20 \mathrm{~mL})$, dried over 
$\mathrm{MgSO}_{4}$ and concentrated in vacuum. The resulting oil was purified by column chromatography (pentane $/ \mathrm{CH}_{2} \mathrm{Cl}_{2} 98 / 2$ to $\left.95 / 5\right)$ to afford $\mathbf{6 h}(61 \mathrm{mg}, 0.12 \mathrm{mmol}, 30 \%)$ as a yellow oil and a mixture of $\mathbf{6 h}$ and $\mathbf{5 h}(25 \mathrm{mg}, 42 \mathrm{w} \%$ product and $58 \mathrm{w} \%$ of starting material, $5 \%$ product and $11 \%$ recovered starting material) as well as $\mathbf{5 h}$ (45 mg, $0.14 \mathrm{mmol}, 35 \%$ ). Total yield: $35 \%$. Yield brsm $=65 \%$. $\mathrm{R}_{\mathrm{f}}$ pentane $/ \mathrm{CH}_{2} \mathrm{Cl}_{2}$ 98/2: 0.15. ${ }^{1} \mathrm{H}$ NMR (400 MHz, $\left.\mathrm{CDCl}_{3}\right) \delta 8.52(\mathrm{~m}, 1 \mathrm{H}, \mathrm{ArH}), 8.42(\mathrm{~m}, 1 \mathrm{H}, \mathrm{ArH}), 7.63-7.51(\mathrm{~m}, 3 \mathrm{H}, \mathrm{ArH})$, 7.32-7.18 (m, $10 \mathrm{H}, \mathrm{ArH}), 6.84$ (d, $1 \mathrm{H}, J=7.8 \mathrm{~Hz}, \mathrm{ArH}), 4.33$ (s, $\left.4 \mathrm{H}, \mathrm{CH}_{2}\right), 1.19$ (m, $\left.21 \mathrm{H}, \mathrm{TIPS}\right) .{ }^{13} \mathrm{C}$ NMR $\left(101 \mathrm{MHz}, \mathrm{CDCl}_{3}\right) \delta 148.2,137.8,134.9,131.0,129.2,128.4,128.3,127.0,127.0,126.7,125.9$, 124.0, 117.7, 116.1, 105.4, 94.5, 57.1, 18.8, 11.4. IR 3067 (w), 3028 (w), 2942 (m), $2872(\mathrm{~m}), 2864(\mathrm{~m})$, $2247(\mathrm{w}), 2143(\mathrm{~m}), 1574(\mathrm{~m}), 1495(\mathrm{w}), 1455(\mathrm{~m}), 1391(\mathrm{~m}), 1363(\mathrm{w}), 1323(\mathrm{w}), 1272(\mathrm{w}), 1218(\mathrm{w}), 1155$ (w), $1068(\mathrm{~m}), 1030$ (w), 992 (w), 908 (s), 884 (m), 832 (w), 767 (s), 734 (s), 698 (s), 673 (s). HRMS (ESI) calcd for $\mathrm{C}_{35} \mathrm{H}_{42} \mathrm{NSi}^{+}[\mathrm{M}+\mathrm{H}]^{+}$504.3081; found 504.3090.

\section{$N, N$-dibenzyl-3-methyl-4-((triisopropylsilyl)ethynyl)aniline (6i)}

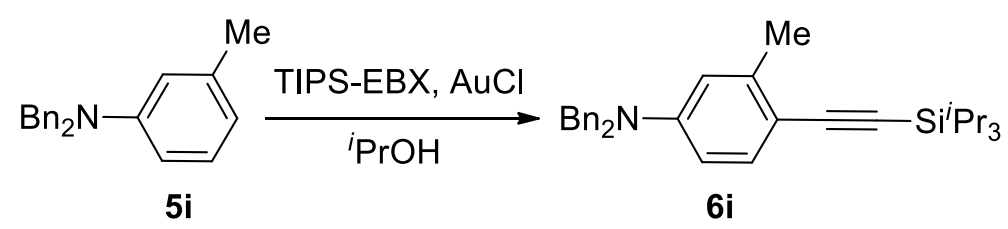

$\mathrm{AuCl}$ (4.6 mg, $0.020 \mathrm{mmol}, 0.05$ equiv) was added to a stirring solution of $\mathbf{5 i}$ ( $115 \mathrm{mg}, 0.400 \mathrm{mmol}, 1$ equiv) and TIPS-EBX (1) (240 mg, $0.560 \mathrm{mmol}, 1.4$ equiv) in ${ }^{i} \mathrm{PrOH}^{8}(2 \mathrm{~mL})$ under air. The reaction was stirred at $60{ }^{\circ} \mathrm{C}$ for $24 \mathrm{~h}$. EtOAc $(20 \mathrm{~mL})$ was added to the reaction mixture. The organic mixture was then washed with $0.1 \mathrm{M} \mathrm{NaOH}(20 \mathrm{~mL})$, a saturated solution of $\mathrm{NaHCO}_{3}(20 \mathrm{~mL})$ and brine $(20 \mathrm{~mL})$, dried over $\mathrm{MgSO}_{4}$ and concentrated in vacuum. The resulting oil was purified by column chromatography (pentane/ $\mathrm{CH}_{2} \mathrm{Cl}_{2}$ 98/2) to afford $6 \mathbf{i}(136 \mathrm{mg}, 0.291 \mathrm{mmol}, 73 \%)$ as yellow oil. $\mathrm{R}_{\mathrm{f}}$ pentane/ $\mathrm{CH}_{2} \mathrm{Cl}_{2}$ 98/2: 0.2. ${ }^{1} \mathrm{H}$ NMR (400 $\mathrm{MHz}_{\mathrm{CDCl}}$ ) $\delta$ 7.39-7.20 (m, $\left.11 \mathrm{H}, \mathrm{ArH}\right), 6.61$ (d, $\left.1 \mathrm{H}, J=2.4 \mathrm{~Hz}, \mathrm{ArH}\right), 6.52(\mathrm{dd}, 1 \mathrm{H}, J=8.6,2.6 \mathrm{~Hz}$, ArH), 4.67 (s, $\left.4 \mathrm{H}, \mathrm{CH}_{2}\right), 2.38$ (s, $\left.3 \mathrm{H}, \mathrm{Me}\right), 1.14$ (m, $\left.21 \mathrm{H}, \mathrm{TIPS}\right) .{ }^{13} \mathrm{C} \mathrm{NMR}\left(101 \mathrm{MHz}, \mathrm{CDCl}_{3}\right) \delta 149.0$, 141.9, 138.1, 133.6, 128.7, 127.0, 126.5, 112.8, 111.4, 109.6, 106.8, 91.3, 53.8, 21.5, 18.7, 11.4. IR 3063 (w), 3028 (w), 2942 (m), $2890(\mathrm{w}), 2864(\mathrm{~m}), 2140$ (m), 1606 (s), 1550 (w), 1506 (s), $1453(\mathrm{~m}), 1366(\mathrm{w})$, 1360 (m), 1297 (w), 1238 (m), 1202 (w), 1162 (w), 1075 (w), 1030 (w), 997 (w), 962 (w), 909 (m), $884(\mathrm{~m})$, 817 (w), 800 (s), 734 (s). HRMS (ESI) calcd for $\mathrm{C}_{32} \mathrm{H}_{42} \mathrm{NSi}^{+}[\mathrm{M}+\mathrm{H}]^{+} 468.3081$; found 468.3076 .

\section{$N, N$-Dibenzyl-3-methoxy-4-((triisopropylsilyl)ethynyl)aniline (6j)}

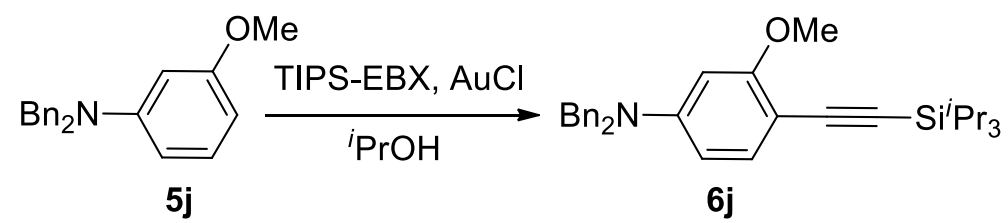

$\mathrm{AuCl}$ (4.6 mg, $0.020 \mathrm{mmol}, 0.05$ equiv) was added to a stirring solution of $\mathbf{5 j}$ ( $121 \mathrm{mg}, 0.400 \mathrm{mmol}, 1$ equiv) and TIPS-EBX (1) (240 mg, $0.560 \mathrm{mmol}, 1.4$ equiv) in ${ }^{i} \mathrm{PrOH}^{8}(8 \mathrm{~mL})$ under air. The reaction was stirred at $60{ }^{\circ} \mathrm{C}$ for $24 \mathrm{~h}$. EtOAc $(20 \mathrm{~mL})$ was added to the reaction mixture. The organic mixture was then washed with $0.1 \mathrm{M} \mathrm{NaOH}(20 \mathrm{~mL})$, a saturated solution of $\mathrm{NaHCO}_{3}(20 \mathrm{~mL})$ and brine $(20 \mathrm{~mL})$, dried over $\mathrm{MgSO}_{4}$ and concentrated in vacuum. The resulting oil was purified by column chromatography (pentane/Et ${ }_{2} \mathrm{O} 200 / 1$ to $100 / 1)$ to afford $\mathbf{6 j}(165 \mathrm{mg}, 0.340 \mathrm{mmol}, 85 \%)$ as a colorless oil. $\mathrm{R}_{\mathrm{f}}$ pentane/Et $\mathrm{t}_{2} \mathrm{O}$ 200/1: 0.2. ${ }^{1} \mathrm{H} \mathrm{NMR}$ $\left(400 \mathrm{MHz}, \mathrm{CDCl}_{3}\right) \delta$ 7.40-7.23 (m, $\left.11 \mathrm{H}, \mathrm{ArH}\right), 6.32(\mathrm{dd}, 1 \mathrm{H}, J=8.6,2.4 \mathrm{~Hz}, \mathrm{ArH}), 6.22(\mathrm{~d}, 1 \mathrm{H}, J=2.3$ $\mathrm{Hz}, \mathrm{ArH}), 4.70$ (s, $\left.4 \mathrm{H}, \mathrm{CH}_{2}\right), 3.69$ (s, $\left.3 \mathrm{H}, \mathrm{OMe}\right), 1.16$ (m, $\left.21 \mathrm{H}, \mathrm{TIPS}\right) .{ }^{13} \mathrm{C} \mathrm{NMR}\left(101 \mathrm{MHz}, \mathrm{CDCl}_{3}\right) \delta$ $161.9,150.5,138.0,134.6,128.7,127.1,126.6,104.8,104.3,101.2,96.3,91.8,55.7,54.5,18.7,11.5$. IR 
3066 (w), 3062 (w), 3030 (w), 2940 (m), 2914 (m), 2863 (m), 2145 (m), $1716(\mathrm{w}), 1608$ (s), $1552(\mathrm{w}), 1515$ (s), $1453(\mathrm{~m}), 1394(\mathrm{w}), 1360(\mathrm{~m}), 1272(\mathrm{~m}), 1268(\mathrm{~m}), 1242(\mathrm{~m}), 1205$ (s), $1165(\mathrm{~m}), 1134(\mathrm{w}), 1075(\mathrm{w})$, 1031 (m), 996 (w), 965 (m), 909 (m), 883 (m), 812 (s), 733 (s), 697 (s), 677 (m). HRMS (ESI) calcd for $\mathrm{C}_{32} \mathrm{H}_{42} \mathrm{NOSi}^{+}[\mathrm{M}+\mathrm{H}]^{+}$484.3030; found 484.3030.

\section{$N, N$-Dibenzyl-3-chloro-4-((triisopropylsilyl)ethynyl)aniline (6k)}

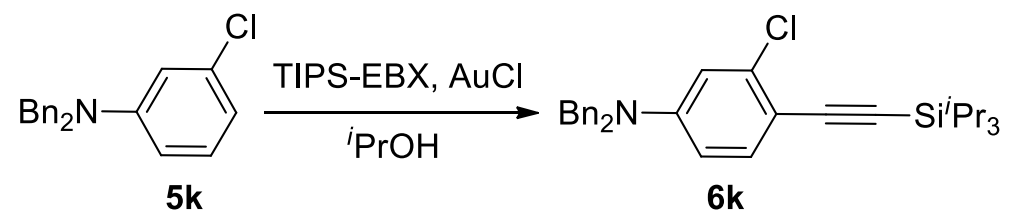

$\mathrm{AuCl}$ (4.6 mg, $0.020 \mathrm{mmol}, 0.05$ equiv) was added to a stirring solution of 5k (123 mg, $0.400 \mathrm{mmol}, 1$ equiv) and TIPS-EBX (1) (240 mg, $0.560 \mathrm{mmol}, 1.4$ equiv) in ${ }^{i} \mathrm{PrOH}^{8}$ (2 mL) under air. The reaction was stirred at $60{ }^{\circ} \mathrm{C}$ for $24 \mathrm{~h}$. EtOAc $(20 \mathrm{~mL})$ was added to the reaction mixture. The organic mixture was then washed with $0.1 \mathrm{M} \mathrm{NaOH}(20 \mathrm{~mL})$, saturated solution of $\mathrm{NaHCO}_{3}(20 \mathrm{~mL})$ and brine $(20 \mathrm{~mL})$, dried over $\mathrm{MgSO}_{4}$ and concentrated in vacuum. The resulting oil was purified by column chromatography (pentane $/ \mathrm{CH}_{2} \mathrm{Cl}_{2}$ 98/2) to afford $\mathbf{6 k}(32 \mathrm{mg}, 0.066 \mathrm{mmol}, 16 \%)$ as a yellow oil and a second batch (64 mg, $76 \mathrm{w} \%$ of $\mathbf{6 k}(0.010 \mathrm{mmol})$ and $24 \mathrm{w} \%$ of $\mathbf{5 k}(0.050 \mathrm{mmol}), 25 \%$ of $\mathbf{6 k}$ and $12 \%$ of $\mathbf{5 k}$ recovered $)$ as well as some pure 5k (38 mg, $0.12 \mathrm{mmol}, 31 \%$ ). Total yield: $41 \%$. Yield brsm $=72 \% . \mathrm{R}_{\mathrm{f}}$ pentane $/ \mathrm{CH}_{2} \mathrm{Cl}_{2}$ 98/2: 0.15. ${ }^{1} \mathrm{H}$ NMR $\left(400 \mathrm{MHz}, \mathrm{CDCl}_{3}\right) \delta$ 7.37-7.23 (m, $\left.7 \mathrm{H}, \mathrm{ArH}\right), 7.19(\mathrm{~d}, 4 \mathrm{H}, J=7.0 \mathrm{~Hz}, \mathrm{ArH}), 6.74(\mathrm{~d}, 1$ $\mathrm{H}, J=2.6 \mathrm{~Hz}, \mathrm{ArH}), 6.53(\mathrm{dd}, 1 \mathrm{H}, J=8.8,2.6 \mathrm{~Hz}, \mathrm{ArH}), 4.64\left(\mathrm{~s}, 4 \mathrm{H}, \mathrm{CH}_{2}\right), 1.11(\mathrm{~m}, 21 \mathrm{H}, \mathrm{TIPS}) .{ }^{13} \mathrm{C}$ $\mathrm{NMR}\left(101 \mathrm{MHz}, \mathrm{CDCl}_{3}\right) \delta 149.6,137.5,137.3,134.4,128.8,127.2,126.4,112.4,111.0,110.5,104.0,93.1$, 54.0, 17.9, 11.3. IR $2944(\mathrm{w}), 2875$ (w), 2865 (m), 2154 (w), 1601 (s), 1530 (w), 1506 (s), $1455(\mathrm{~m}), 1397$ (w), $1355(\mathrm{w}), 1296(\mathrm{w}), 1231(\mathrm{~m}), 1203(\mathrm{w}), 1146(\mathrm{w}), 997(\mathrm{w}), 959(\mathrm{w}), 910(\mathrm{~m}), 883(\mathrm{w}), 831(\mathrm{w}), 785$ (m), 734 (s). HRMS (ESI) calcd for $\mathrm{C}_{31} \mathrm{ClH}_{39} \mathrm{NSi}^{+}[\mathrm{M}+\mathrm{H}]^{+} 488.2535$; found 488.2529.

\section{$N, N$-Dibenzyl-3-bromo-4-((triisopropylsilyl)ethynyl)aniline (61)}

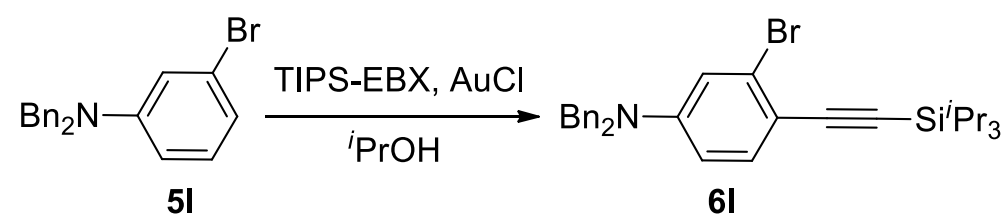

$\mathrm{AuCl}$ (4.6 mg, $0.020 \mathrm{mmol}, 0.05$ equiv) was added to a stirring solution of $\mathbf{5 l}$ (140 $\mathrm{mg}, 0.400 \mathrm{mmol}, 1$ equiv) and TIPS-EBX (1) (240 mg, $0.560 \mathrm{mmol}, 1.4$ equiv) in ${ }^{i} \mathrm{PrOH}^{8}(2 \mathrm{~mL})$ under air. The reaction was stirred at $60{ }^{\circ} \mathrm{C}$ for $24 \mathrm{~h}$. EtOAc $(20 \mathrm{~mL})$ was added to the reaction mixture. The organic mixture was then washed with $0.1 \mathrm{M} \mathrm{NaOH}(20 \mathrm{~mL})$, a saturated solution of $\mathrm{NaHCO}_{3}(20 \mathrm{~mL})$ and brine $(20 \mathrm{~mL})$, dried over $\mathrm{MgSO}_{4}$ and concentrated in vacuum. The resulting oil was purified by column chromatography (pentane/ $\mathrm{CH}_{2} \mathrm{Cl}_{2}$ 99/1 to 95/5) to afford $6 \mathbf{1}(75 \mathrm{mg}, 0.14 \mathrm{mmol}, 35 \%)$ as a yellow oil and $\mathbf{5 l}$ (59 $\mathrm{mg}, 0.17 \mathrm{mmol}, 41 \%$ recovered). Yield brsm $=61 \%$. $\mathrm{R}_{\mathrm{f}}$ pentane $/ \mathrm{CH}_{2} \mathrm{Cl}_{2}$ 99/1: 0.15. ${ }^{1} \mathrm{H} \mathrm{NMR}\left(400 \mathrm{MHz}, \mathrm{CDCl}_{3}\right) \delta 7.34(\mathrm{~m}, 4 \mathrm{H}$, ArH), 7.28 (m, $3 \mathrm{H}, \mathrm{ArH}), 7.24-7.16(\mathrm{~m}, 4 \mathrm{H}, \mathrm{ArH}), 6.95$ (d, $1 \mathrm{H}, J=2.6 \mathrm{~Hz}, \mathrm{ArH}), 6.57$ (dd, $1 \mathrm{H}, J=8.7$, $2.6 \mathrm{~Hz}, \mathrm{ArH}), 4.63$ (s, $\left.4 \mathrm{H}, \mathrm{CH}_{2}\right), 1.13$ (m, $\left.21 \mathrm{H}, \mathrm{TIPS}\right) .{ }^{13} \mathrm{C} \mathrm{NMR}\left(101 \mathrm{MHz}, \mathrm{CDCl}_{3}\right) \delta 149.6,137.3,134.5$, 128.8, 127.3, 127.1, 126.5, 115.5, 113.3, 111.1, 105.7, 92.5, 54.0, 18.7, 11.4. IR 2944 (w), 2865 (w), 2251 (w), $2153(\mathrm{w}), 1726(\mathrm{w}), 1598(\mathrm{~m}), 1502(\mathrm{~m}), 1454(\mathrm{w}), 1230(\mathrm{w}), 1140(\mathrm{w}), 1030(\mathrm{w}), 997(\mathrm{w}), 958(\mathrm{w})$, 906 (s), $884(w), 856$ (w). HRMS (ESI) calcd for $\mathrm{C}_{31}{ }^{79} \mathrm{BrH}_{39} \mathrm{NSi}^{+}[\mathrm{M}+\mathrm{H}]^{+}$532.2030; found 532.2019.

\section{$N, N$-Diethyl-3-methyl-4-((triisopropylsilyl)ethynyl)aniline (6m)}




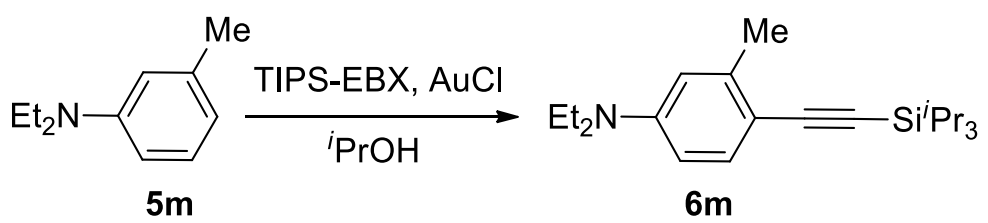

$\mathrm{AuCl}$ (4.6 mg, $0.020 \mathrm{mmol}, 0.05$ equiv) was added to a stirring solution of $\mathbf{5 m}$ ( $71 \mu \mathrm{L}, 0.40 \mathrm{mmol}, 1$ equiv) and TIPS-EBX (1) (240 mg, $0.560 \mathrm{mmol}, 1.4$ equiv) in ${ }^{i} \mathrm{PrOH}^{8}(2 \mathrm{~mL})$ under air. The reaction was stirred at $60^{\circ} \mathrm{C}$ for $24 \mathrm{~h}$. EtOAc $(20 \mathrm{~mL})$ was added to the reaction mixture. The organic mixture was then washed with $0.1 \mathrm{M} \mathrm{NaOH}(20 \mathrm{~mL})$, a saturated solution of $\mathrm{NaHCO}_{3}(20 \mathrm{~mL})$ and brine $(20 \mathrm{~mL})$, dried over $\mathrm{MgSO}_{4}$ and concentrated in vacuum. The resulting oil was purified by column chromatography (pentane/ $\mathrm{CH}_{2} \mathrm{Cl}_{2}$ 98/2 to $95 / 5)$ to afford $\mathbf{6 m}\left(103 \mathrm{mg}, 0.300 \mathrm{mmol}, 75 \%, 95 \%\right.$ pure) as a yellow oil. $\mathrm{R}_{\mathrm{f}}$ pentane $/ \mathrm{CH}_{2} \mathrm{Cl}_{2}$ 98/2: 0.15. ${ }^{1} \mathrm{H}$ NMR $\left(400 \mathrm{MHz}, \mathrm{CDCl}_{3}\right) \delta 7.33(\mathrm{~d}, 1 \mathrm{H}, J=8.6 \mathrm{~Hz}, \mathrm{ArH}), 6.50(\mathrm{~d}, 1 \mathrm{H}, J=2.5 \mathrm{~Hz}, \mathrm{ArH}), 6.45(\mathrm{dd}$, $1 \mathrm{H}, J=8.6,2.6 \mathrm{~Hz}, \mathrm{ArH}), 3.38$ (q, $\left.4 \mathrm{H}, J=7.1 \mathrm{~Hz}, \mathrm{CH}_{2}\right), 2.45$ (s, $\left.3 \mathrm{H}, \mathrm{CH}_{3}\right), 1.15\left(\mathrm{~m}, 27 \mathrm{H}, \mathrm{CH}_{3}+\mathrm{TIPS}\right)$. ${ }^{13} \mathrm{C} \mathrm{NMR}\left(101 \mathrm{MHz}, \mathrm{CDCl}_{3}\right) \delta 147.5,141.8,133.6,112.1,109.7,108.8,107.3,90.6,44.3,21.6,18.7,12.6$, 11.4. IR 2965 (m), 2941 (m), 2918 (m), 2864 (m), 2141 (m), 1713 (w), 1607 (s), 1548 (w), 1507 (m), 1464 (m), 1377 (w), 1256 (m), 1198 (w), 1113 (w), 1076 (w), 1017 (w), 996 (w), 909 (m), 881 (m), 860 (w), 840 $(\mathrm{w}), 793(\mathrm{~s}), 735(\mathrm{~m}), 695(\mathrm{~m}), 677(\mathrm{~s})$. HRMS (ESI) calcd for $\mathrm{C}_{22} \mathrm{H}_{38} \mathrm{NSi}^{+}[\mathrm{M}+\mathrm{H}]^{+}$344.2768; found 344.2768 .

$N, N$-Dibenzyl-4-methyl-2-((triisopropylsilyl)ethynyl)aniline (25)

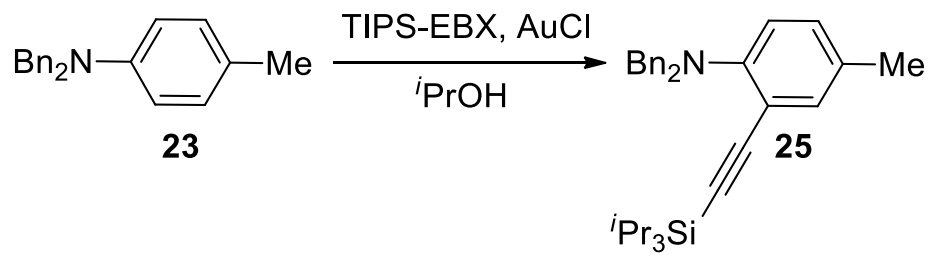

$\mathrm{AuCl}$ (4.6 mg, $0.020 \mathrm{mmol}, 0.05$ equiv) was added to a stirring solution of aniline 23 (115 mg, $0.400 \mathrm{mmol}$, 1 equiv) and TIPS-EBX (1) (240 mg, $0.560 \mathrm{mmol}, 1.4$ equiv) in ${ }^{i} \mathrm{PrOH}^{8}(2 \mathrm{~mL})$ under air. The reaction was stirred at $60{ }^{\circ} \mathrm{C}$ for $24 \mathrm{~h}$. EtOAc $(20 \mathrm{~mL})$ was added to the reaction mixture. The organic mixture was then washed with $0.1 \mathrm{M} \mathrm{NaOH}(20 \mathrm{~mL})$, a saturated solution of $\mathrm{NaHCO}_{3}(20 \mathrm{~mL})$ and brine $(20 \mathrm{~mL})$, dried over $\mathrm{MgSO}_{4}$ and concentrated in vacuum. The resulting oil was purified by column chromatography (pentane/ $\mathrm{CH}_{2} \mathrm{Cl}_{2}$ 95/5) to afford 25 (34 mg, $0.073 \mathrm{mmol}, 18 \%$ ) as a colorless oil. $\mathrm{Rf}$ (pentane $/ \mathrm{CH}_{2} \mathrm{Cl}_{2}$ 95/5) 0.15. ${ }^{1} \mathrm{H}$ NMR (400 MHz, $\left.\mathrm{CDCl}_{3}\right) \delta 7.35(\mathrm{~d}, 1 \mathrm{H}, J=2.0 \mathrm{~Hz}, \mathrm{ArH}), 7.31-7.21$ (m, $\left.10 \mathrm{H}, \mathrm{ArH}\right), 6.95$ (ddd, 1 $\mathrm{H}, J=8.3,2.2,0.6 \mathrm{~Hz}, \mathrm{ArH}), 6.67(\mathrm{~d}, 1 \mathrm{H}, J=8.3 \mathrm{~Hz}, \mathrm{ArH}), 4.38\left(\mathrm{~s}, 4 \mathrm{H}, \mathrm{CH}_{2}\right), 2.27\left(\mathrm{~s}, 3 \mathrm{H}, \mathrm{CH}_{3}\right), 1.16(\mathrm{~m}$, $21 \mathrm{H}, \mathrm{TIPS}) .{ }^{13} \mathrm{C}$ NMR $\left(101 \mathrm{MHz}, \mathrm{CDCl}_{3}\right) \delta 150.6,138.4,135.4,130.8,129.7,128.6,128.1,126.8,121.3$, 117.6, 106.3, 95.4, 55.6, 20.4, 18.8, 11.5. IR 3064 (w), 3027 (w), 2940 (m), 2863 (w), 2145 (w), 1602 (m), 1570 (w), 1496 (s), 1455 (m), 1344 (w), 1305 (m), 1278 (w), 1192 (w), 1154 (w), 1077 (w), $1021(w), 883$ (w), $779(\mathrm{w}), 742(\mathrm{~s}), 700(\mathrm{~s}), 646(\mathrm{~m})$. HRMS (ESI) calcd for $\mathrm{C}_{32} \mathrm{H}_{42} \mathrm{NSi}^{+}[\mathrm{M}+\mathrm{H}]^{+}$468.3081; found 468.3081 .

$N, 4-D i m e t h y l-N$-(3-(triisopropylsilyl)prop-2-yn-1-yl)aniline (7)
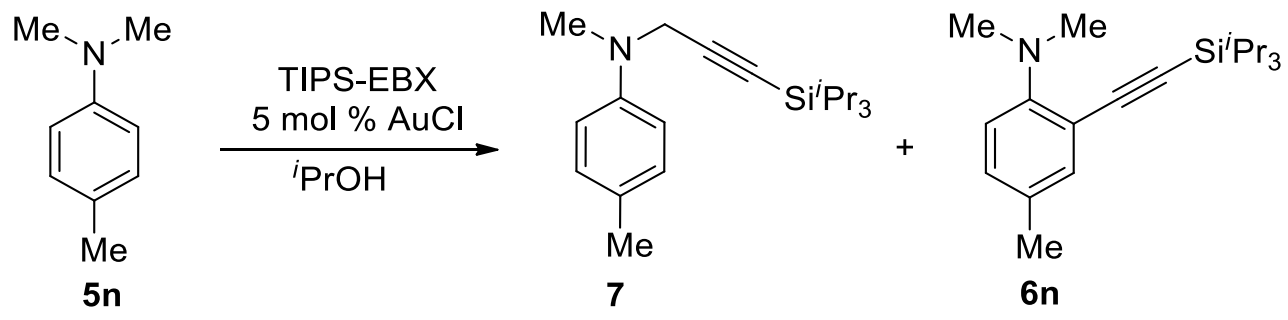
$\mathrm{AuCl}$ (4.6 mg, $0.020 \mathrm{mmol}, 0.05$ equiv) was added to a stirring solution of aniline $5 \mathbf{n}$ (91 mg, $0.40 \mathrm{mmol}, 1$ equiv) and TIPS-EBX (1) $\left(240 \mathrm{mg}, 0.560 \mathrm{mmol}, 1.4\right.$ equiv) in ${ }^{i} \mathrm{PrOH}^{8}(2 \mathrm{~mL})$ under $\mathrm{N}_{2}$. The reaction was stirred at $60{ }^{\circ} \mathrm{C}$ for $24 \mathrm{~h}$. EtOAc $(20 \mathrm{~mL})$ was added to the reaction mixture. The organic mixture was then washed with $0.1 \mathrm{M} \mathrm{NaOH}(20 \mathrm{~mL})$, a saturated solution of $\mathrm{NaHCO}_{3}(20 \mathrm{~mL})$ and brine $(20 \mathrm{~mL})$, dried over $\mathrm{MgSO}_{4}$ and concentrated in vacuum. The resulting oil was purified by column chromatography (pentane/ $\mathrm{CH}_{2} \mathrm{Cl}_{2}$ 9/1 to 8/2) to afford 7 (28 mg, $0.089 \mathrm{mmol}, 22 \%$ ) as a yellow oil and traces of $\mathbf{6 n}$ were identified by ${ }^{1} \mathrm{H}$ NMR. $\mathrm{R}_{\mathrm{f}}$ (pentane/ $\left.\mathrm{CH}_{2} \mathrm{Cl}_{2} 8 / 2\right)$ 0.2. ${ }^{1} \mathrm{H} \mathrm{NMR}\left(400 \mathrm{MHz}, \mathrm{CDCl}_{3}\right) \delta 7.06(\mathrm{~d}, 2 \mathrm{H}, J=8.7 \mathrm{~Hz}$, ArH), $6.83(\mathrm{~d}, 2 \mathrm{H}, J=8.5 \mathrm{~Hz}, \mathrm{ArH}), 4.05\left(\mathrm{~s}, 2 \mathrm{H}, \mathrm{CH}_{2}\right), 2.94\left(\mathrm{~s}, 3 \mathrm{H}, \mathrm{CH}_{3}\right), 2.28\left(\mathrm{~s}, 3 \mathrm{H}, \mathrm{CH}_{3}\right), 1.02(\mathrm{~m}, 21$ $\mathrm{H}, \mathrm{TIPS}) .{ }^{13} \mathrm{C} \mathrm{NMR}\left(101 \mathrm{MHz}, \mathrm{CDCl}_{3}\right) \delta 147.4,129.4,127.7,115.4,103.2,85.0,44.2,38.9,20.3,18.5$, 11.1. IR 3012 (w), 2942 (s), 2865 (s), 2808 (w), 2166 (w), 1861 (w), 1619 (m), 1519 (s), 1463 (m), 1371 (w), 1363 (m), 1329 (m), $1238(\mathrm{~m}), 1191$ (m), $1109(\mathrm{~m}), 1074$ (w), 1000 (s), 976 (m), 923 (m), 883 (s), 807 (s), $738(\mathrm{w}), 665$ (s), $643(\mathrm{~m})$. HRMS (ESI) calcd for $\mathrm{C}_{20} \mathrm{H}_{34} \mathrm{NSi}^{+}[\mathrm{M}+\mathrm{H}]^{+}$316.2455; found 316.2443.

\section{Triisopropyl((2,4,6-trimethoxyphenyl)ethynyl)silane (8a)}

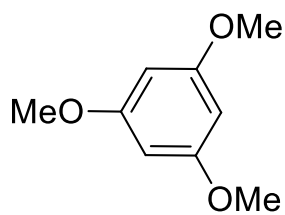

26

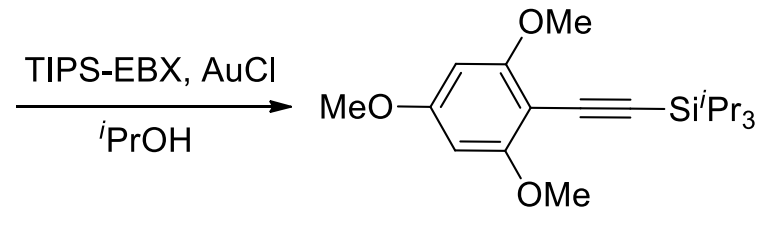

$8 \mathbf{a}$

$\mathrm{AuCl}$ (2.3 mg, $0.010 \mathrm{mmol}, 0.05$ equiv) was added to a stirring solution of trimethoxybenzene (26) (34 mg, $0.20 \mathrm{mmol}, 1$ equiv) and TIPS-EBX (1) (120 mg, $0.280 \mathrm{mmol}, 1.4$ equiv) in ${ }^{i} \mathrm{PrOH}^{8}(4 \mathrm{~mL})$ under air. The reaction was stirred at RT for $20 \mathrm{~h}$. EtOAc $(20 \mathrm{~mL})$ was added to the reaction mixture. The organic mixture was then washed with $0.1 \mathrm{~m} \mathrm{NaOH}(20 \mathrm{~mL})$, a saturated solution of $\mathrm{NaHCO}_{3}(20 \mathrm{~mL})$ and brine $(20 \mathrm{~mL})$, dried over $\mathrm{MgSO}_{4}$ and concentrated in vacuum. The resulting oil was purified by column chromatography (pentane/Et $2 \mathrm{O} 95 / 5)$ to afford $\mathbf{8 a}(35 \mathrm{mg}, 0.10 \mathrm{mmol}, 51 \%)$ as a colorless oil and 26 (12 $\mathrm{mg}, 0.071 \mathrm{mmol}$, $36 \%$ recovered). Yield brsm $=80 \% . \mathrm{R}_{\mathrm{f}}\left(\right.$ pentane/Et $\left._{2} \mathrm{O} 95 / 5\right)$ 0.2. ${ }^{1} \mathrm{H} \mathrm{NMR}\left(400 \mathrm{MHz}, \mathrm{CDCl}_{3}\right) \delta 6.08(\mathrm{~s}, 2 \mathrm{H}$, $\mathrm{ArH}), 3.85$ (s, $6 \mathrm{H}, \mathrm{OMe}), 3.83$ (s, $3 \mathrm{H}, \mathrm{OMe}), 1.17$ (m, $21 \mathrm{H}, \mathrm{TIPS}) .{ }^{13} \mathrm{C} \mathrm{NMR}\left(101 \mathrm{MHz}, \mathrm{CDCl}_{3}\right) \delta 163.0$, 161.3, 99.1, 97.5, 95.4, 90.6, 56.0, 55.3, 18.7, 11.5. IR 2944 (m), $2864(\mathrm{~m}), 2147(\mathrm{~m}), 1603(\mathrm{~s}), 1463$ (s), 1419 (w), 1340 (w), 1216 (s), 1132 (s), 1053 (w), 999 (w), 914 (w), 885 (w), 819 (s), 735 (w), 666 (m). HRMS (ESI) calcd for $\mathrm{C}_{20} \mathrm{H}_{33} \mathrm{O}_{3} \mathrm{Si}^{+}[\mathrm{M}+\mathrm{H}]^{+}$349.2193; found 349.2191.

\section{Triisopropyl((2,4,6-trimethoxy-3-methylphenyl)ethynyl)silane $(8 b)$}

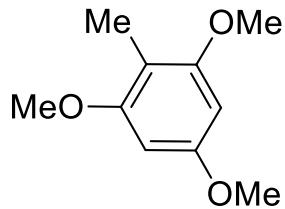

27

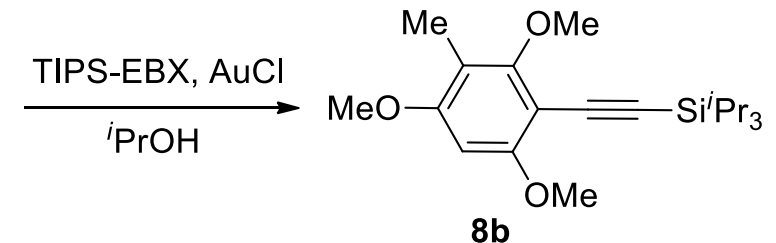

8b

$\mathrm{AuCl}$ (4.6 mg, $0.020 \mathrm{mmol}, 0.05$ equiv) was added to a stirring solution of trimethoxytoluene (7m) $(73 \mathrm{mg}$, $0.40 \mathrm{mmol}, 1$ equiv) and TIPS-EBX (1) $\left(240 \mathrm{mg}, 0.560 \mathrm{mmol}, 1.4\right.$ equiv) in ${ }^{i} \mathrm{PrOH}^{8}(8 \mathrm{~mL})$ under air. The reaction was stirred at $\mathrm{RT}$ for $36 \mathrm{~h}$. EtOAc $(20 \mathrm{~mL})$ was added to the reaction mixture. The organic mixture was then washed with $0.1 \mathrm{M} \mathrm{NaOH}(20 \mathrm{~mL})$, a saturated solution of $\mathrm{NaHCO}_{3}(20 \mathrm{~mL})$ and brine $(20 \mathrm{~mL})$, dried over $\mathrm{MgSO}_{4}$ and concentrated in vacuum. The resulting oil was purified by column chromatography (pentane/Et $2 \mathrm{O} 9 / 1)$ to afford a mixture of $\mathbf{8 b}$ and $27(99 \mathrm{mg}, 72 \mathrm{w} \%$ of $\mathbf{8 b}(0.20 \mathrm{mmol})$ and $28 \mathrm{w} \%$ of $27(0.15$ $\mathrm{mmol}$ ), $50 \%$ of $\mathbf{8 b}$ and $37 \%$ of $\mathbf{2 7}$ recovered) as a colorless oil. Yield brsm $=79 \%$. Analytical pure compound was obtained by column chromatography. $\mathrm{R}_{\mathrm{f}}$ (pentane/ $\mathrm{Et}_{2} \mathrm{O}$ 9/1) 0.2. ${ }^{1} \mathrm{H} \mathrm{NMR}(400 \mathrm{MHz}$, 
$\left.\mathrm{CDCl}_{3}\right) \delta 6.23(\mathrm{~s}, 1 \mathrm{H}, \mathrm{ArH}), 3.91(\mathrm{~s}, 3 \mathrm{H}, \mathrm{OMe}), 3.88$ (s, $\left.3 \mathrm{H}, \mathrm{OMe}\right), 3.85$ (s, $\left.3 \mathrm{H}, \mathrm{OMe}\right), 2.07$ (s, $\left.3 \mathrm{H}, \mathrm{Me}\right)$, $1.16\left(\mathrm{~m}, 21 \mathrm{H}\right.$, TIPS). ${ }^{13} \mathrm{C}$ NMR $\left(101 \mathrm{MHz}, \mathrm{CDCl}_{3}\right) \delta 161.1,160.9,159.1,112.1,99.7,99.4,97.6,91.4$, 60.6, 56.3, 55.6, 18.7, 11.5, 8.3. IR 2937 (m), $2864(\mathrm{~m}), 2151(\mathrm{w}), 1729(\mathrm{w}), 1599(\mathrm{~m}), 1579(\mathrm{w}), 1496(\mathrm{w})$, 1463 (m), 1436 (w), 1399 (m), 1330 (m), 1291 (w), 1220 (m), 1191 (w), 1137 (s), 1120 (s), 1019 (m), 997 (w), $919(\mathrm{~m}), 881(\mathrm{~m}), 803(\mathrm{~m}), 745(\mathrm{~m}), 676(\mathrm{~m}), 659(\mathrm{~m})$. HRMS (ESI) calcd for $\mathrm{C}_{21} \mathrm{H}_{34} \mathrm{AgO}_{3} \mathrm{Si}^{+}[\mathrm{M}+\mathrm{Ag}]^{+}$ 469.1323; found 469.1312 .

\section{Spectra of New Compounds}


solvent: <CDCI3 >

Frequency $400.13 \mathrm{MHz}$<smiles>COc1ccccc1NC(C)c1ccccc1</smiles>
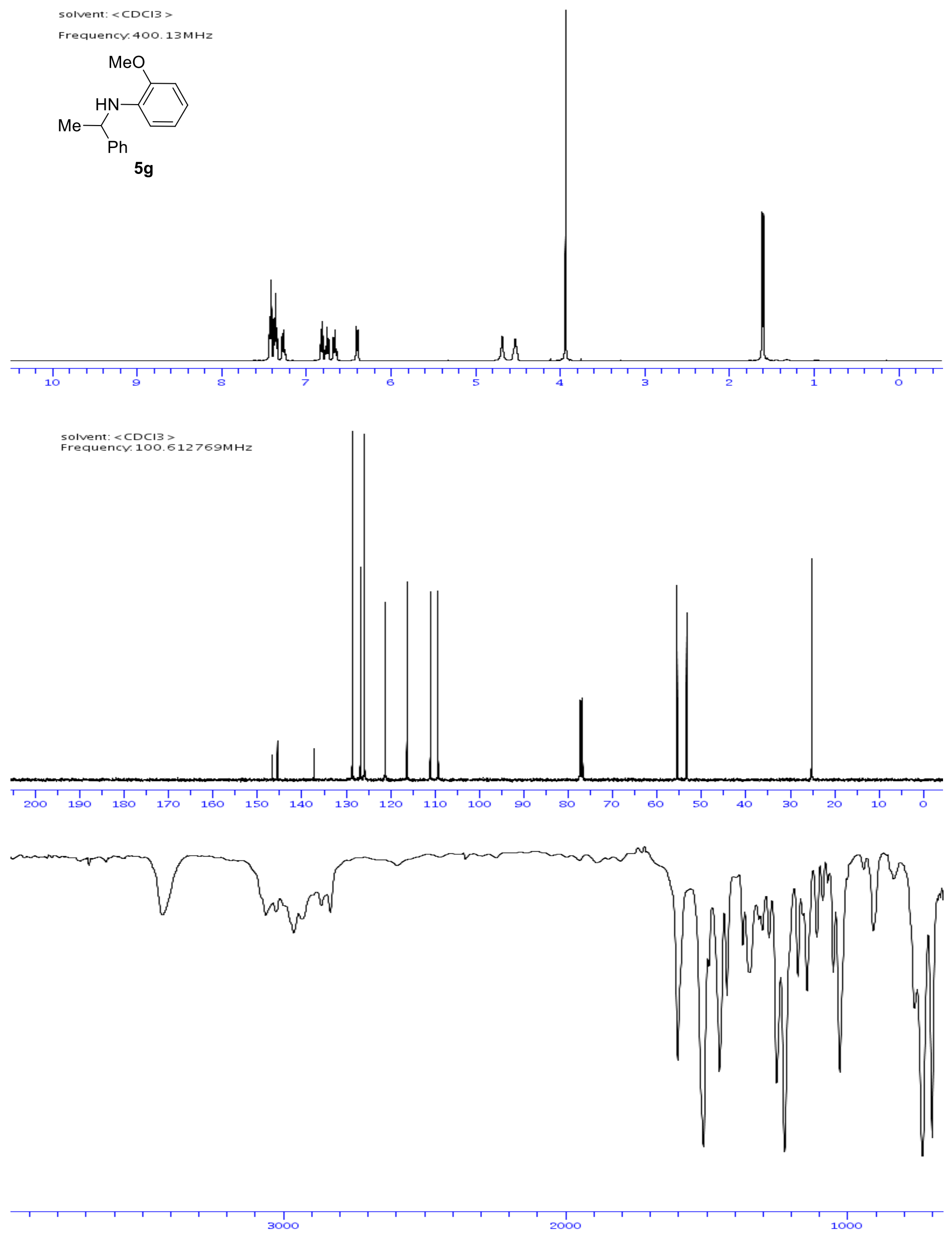

17 

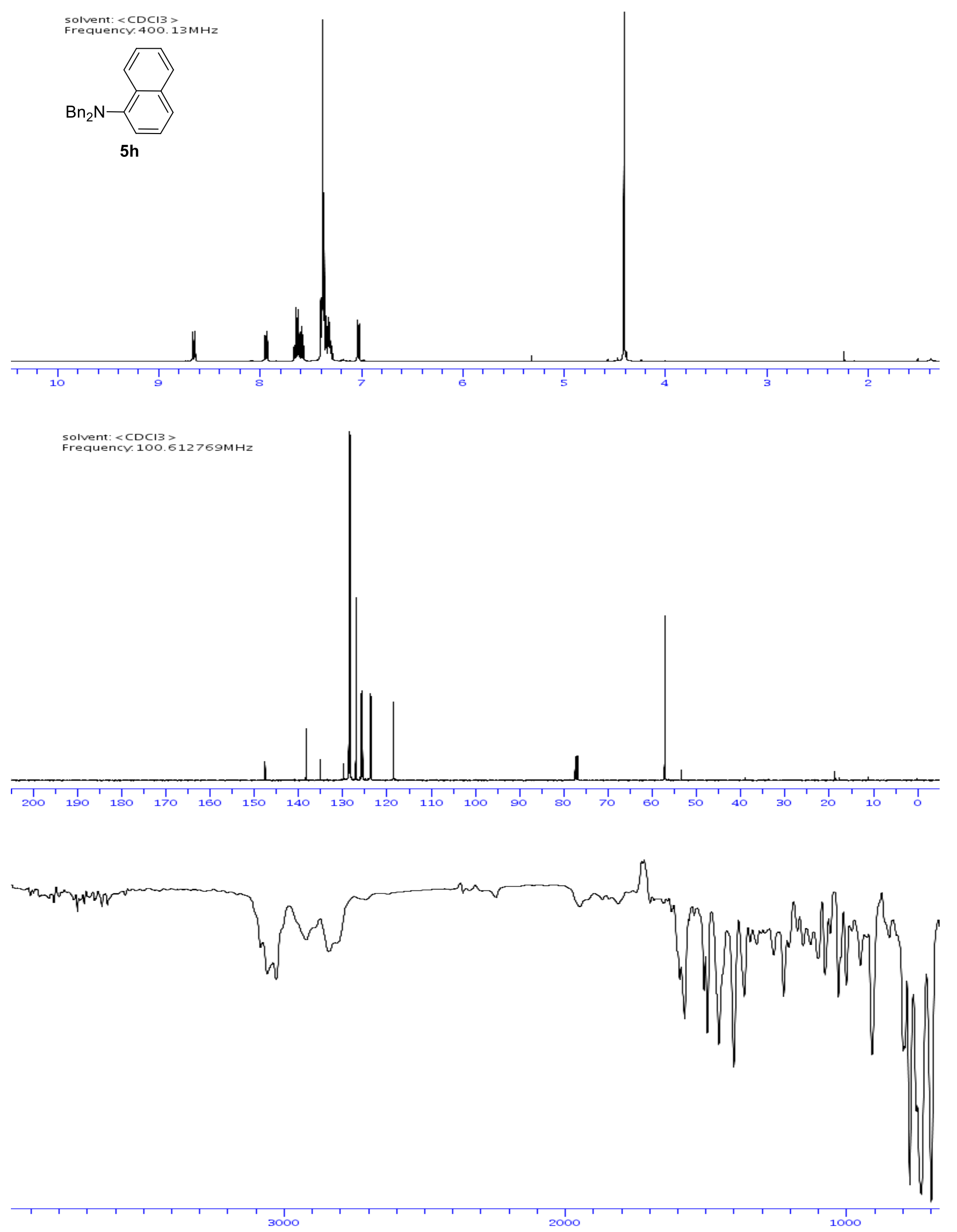
solvent: $<C D C I 3>$

Frequency $400.13 \mathrm{MHz}$

$\mathrm{Bn}_{2} \mathrm{~N}-\mathrm{C}^{\mathrm{Me}}$

$5 \mathbf{i}$

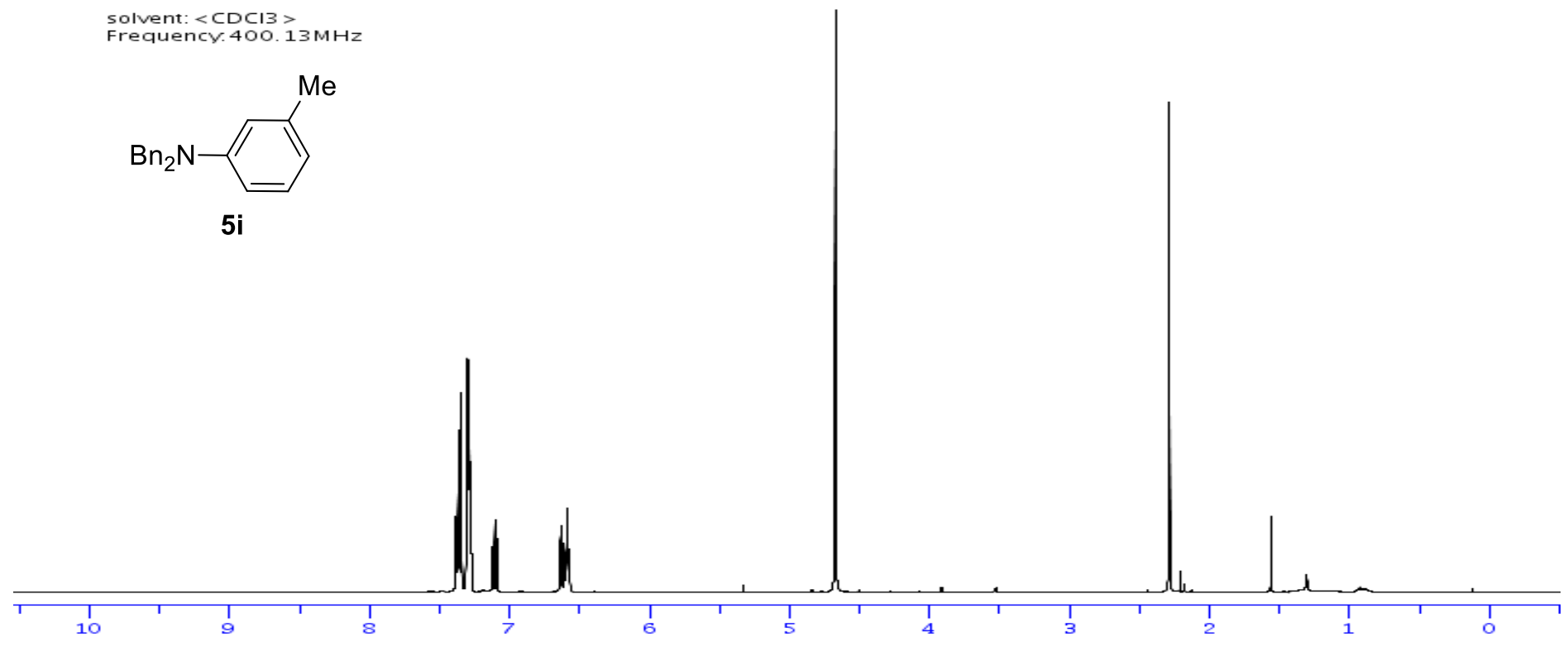

solvent: $<C D C I 3>$
Frequency. $100.612769 \mathrm{MHz}$
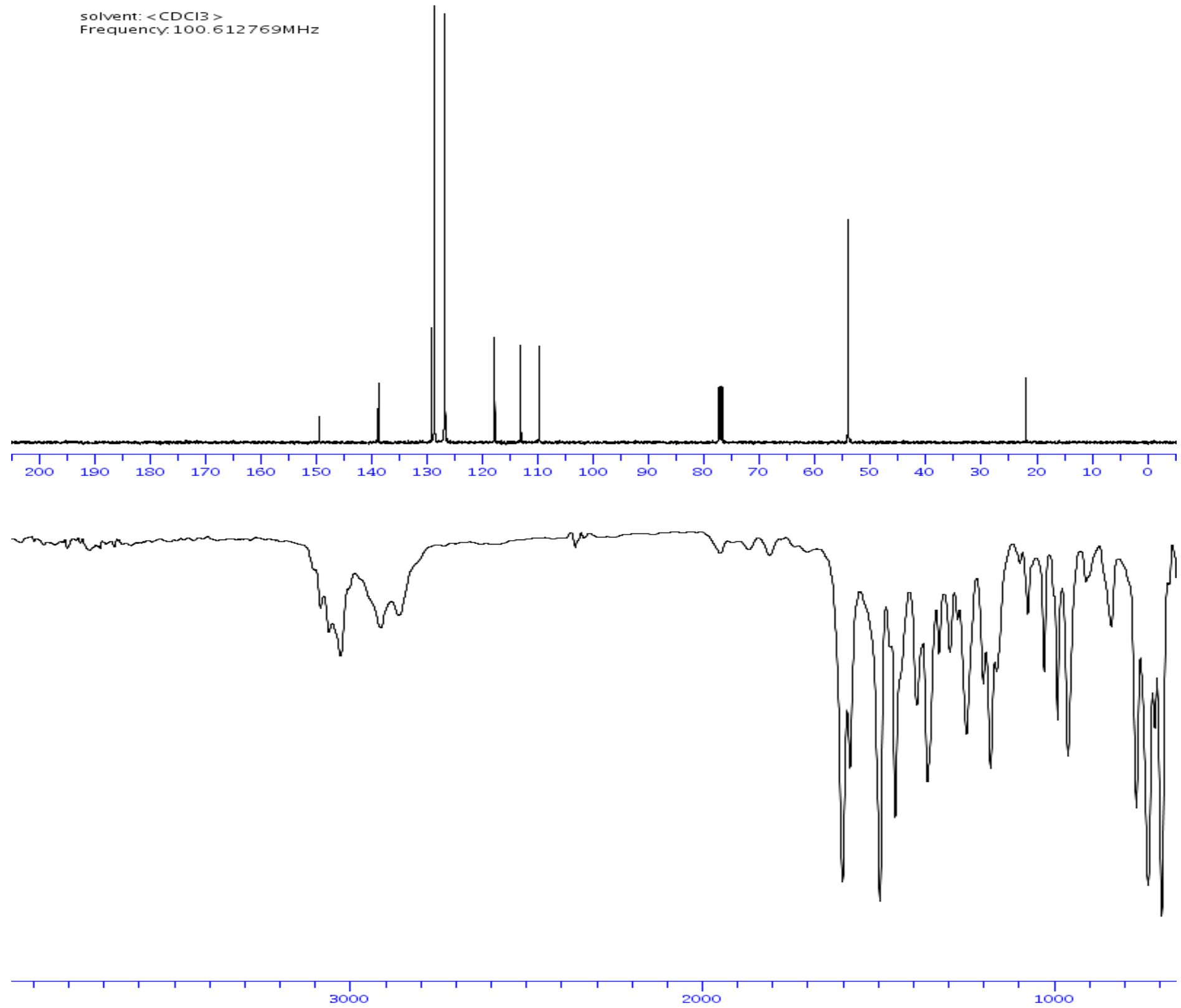
solvent: $<C D C I 3>$

Frequency $400.13 \mathrm{MHz}$

$\mathrm{OMe}$

$\mathrm{Bn}_{2} \mathrm{~N}$

$5 j$

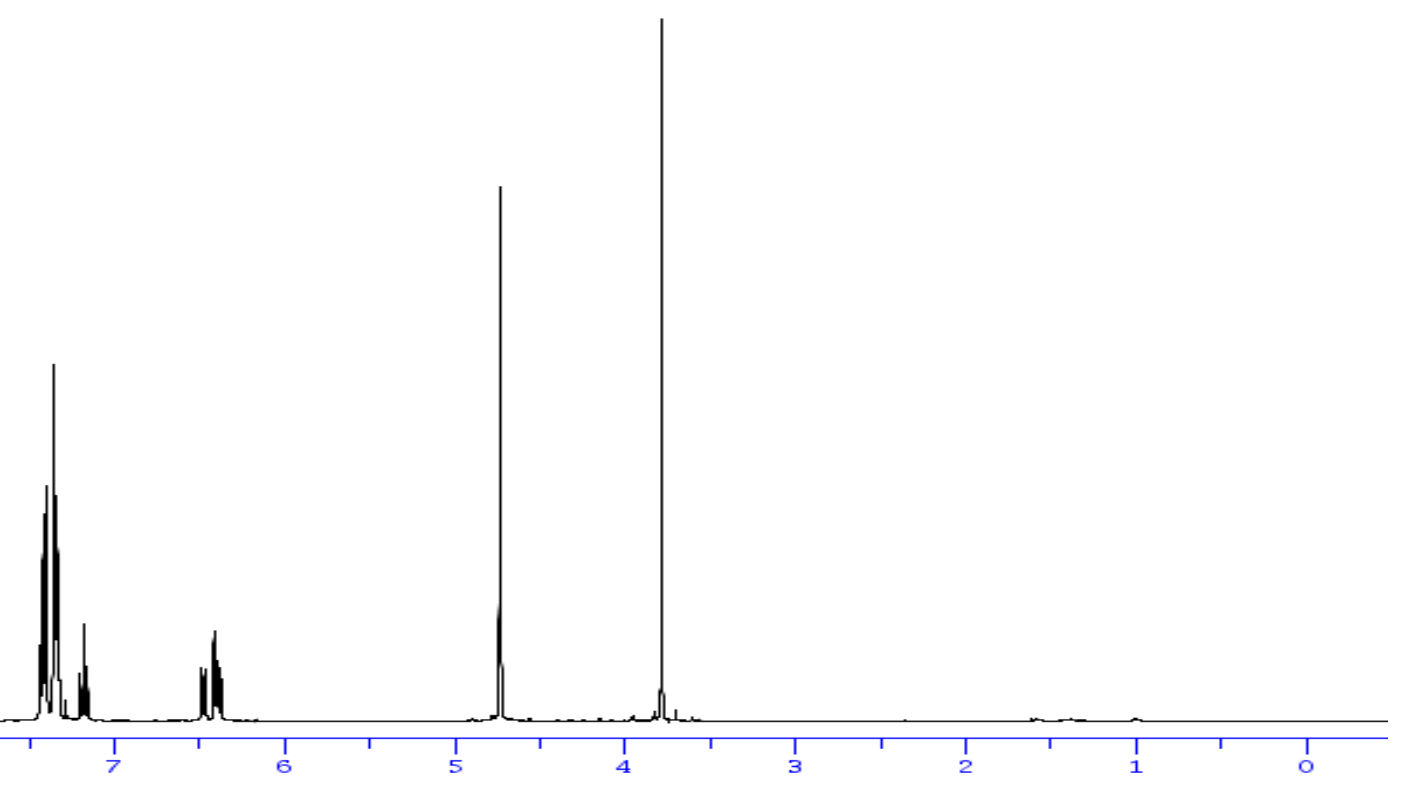

Frequency. $100.612769 \mathrm{MHz}$
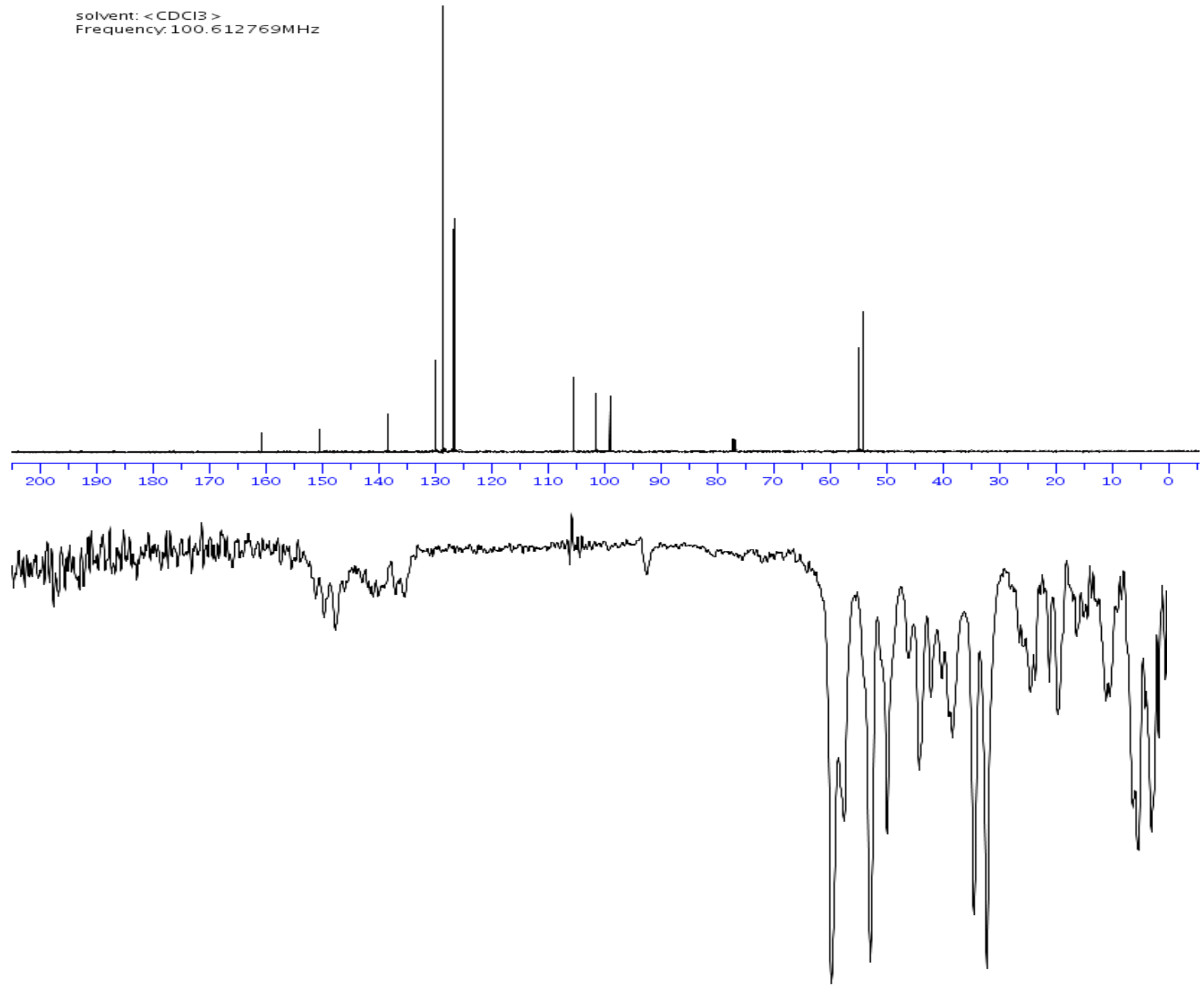

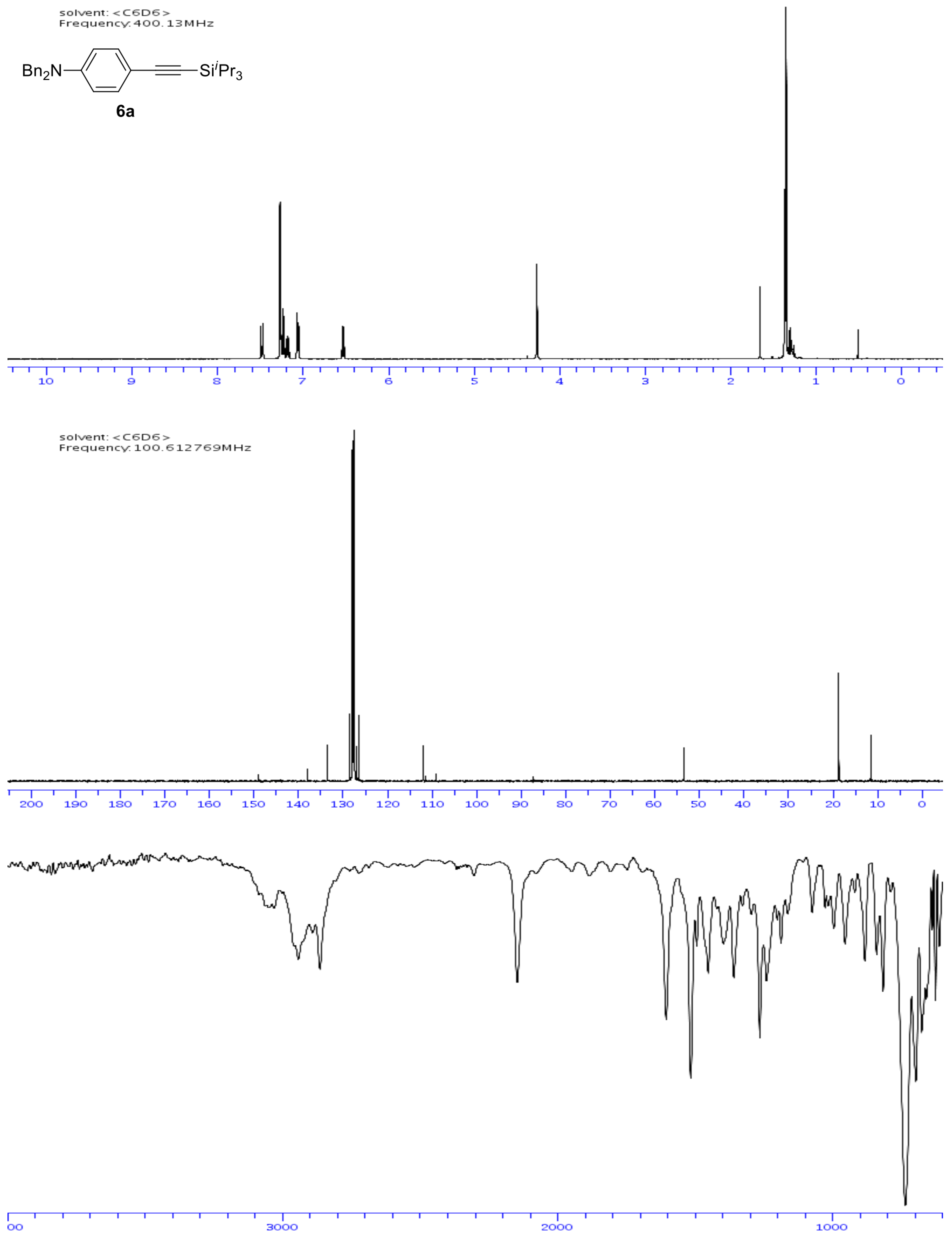

21 


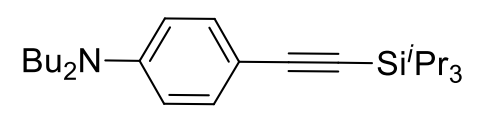

6b
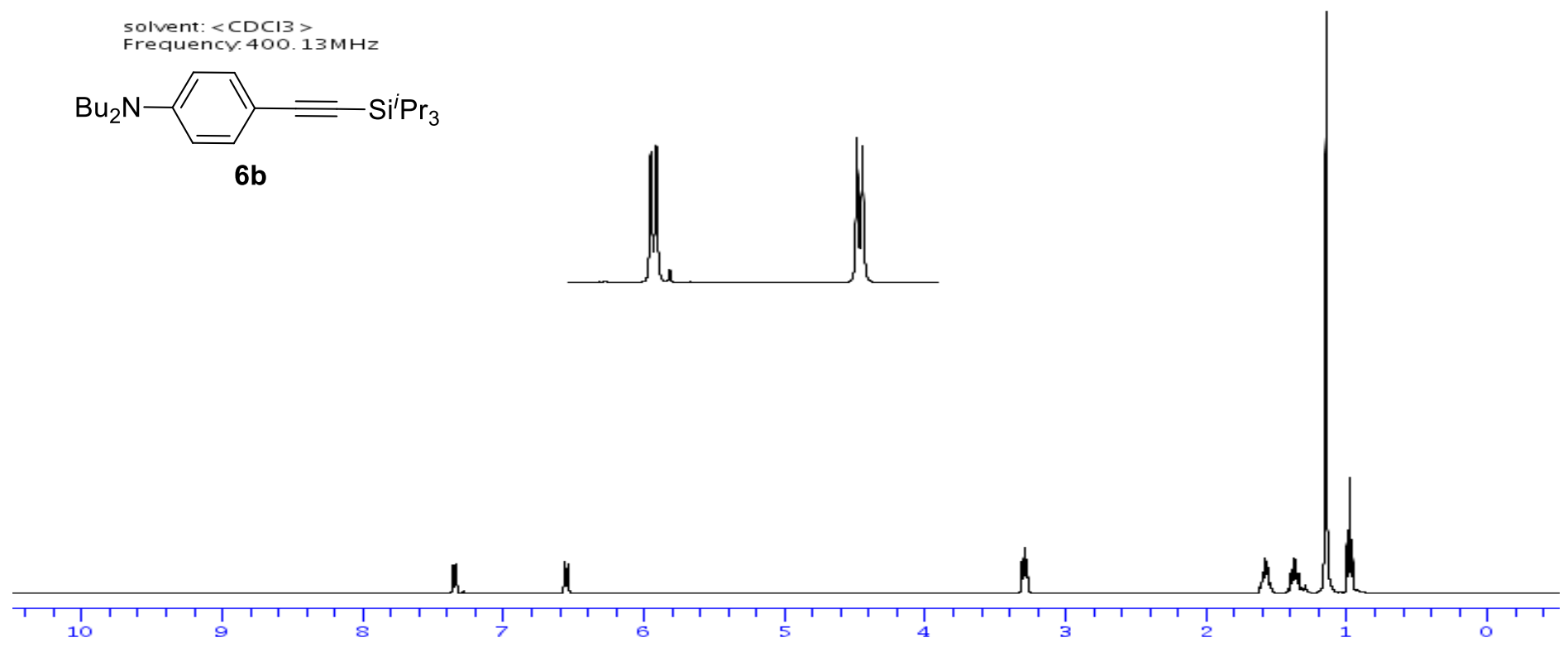

solvent: <CDCI3 >

Frequency. $100.612769 \mathrm{MHz}$
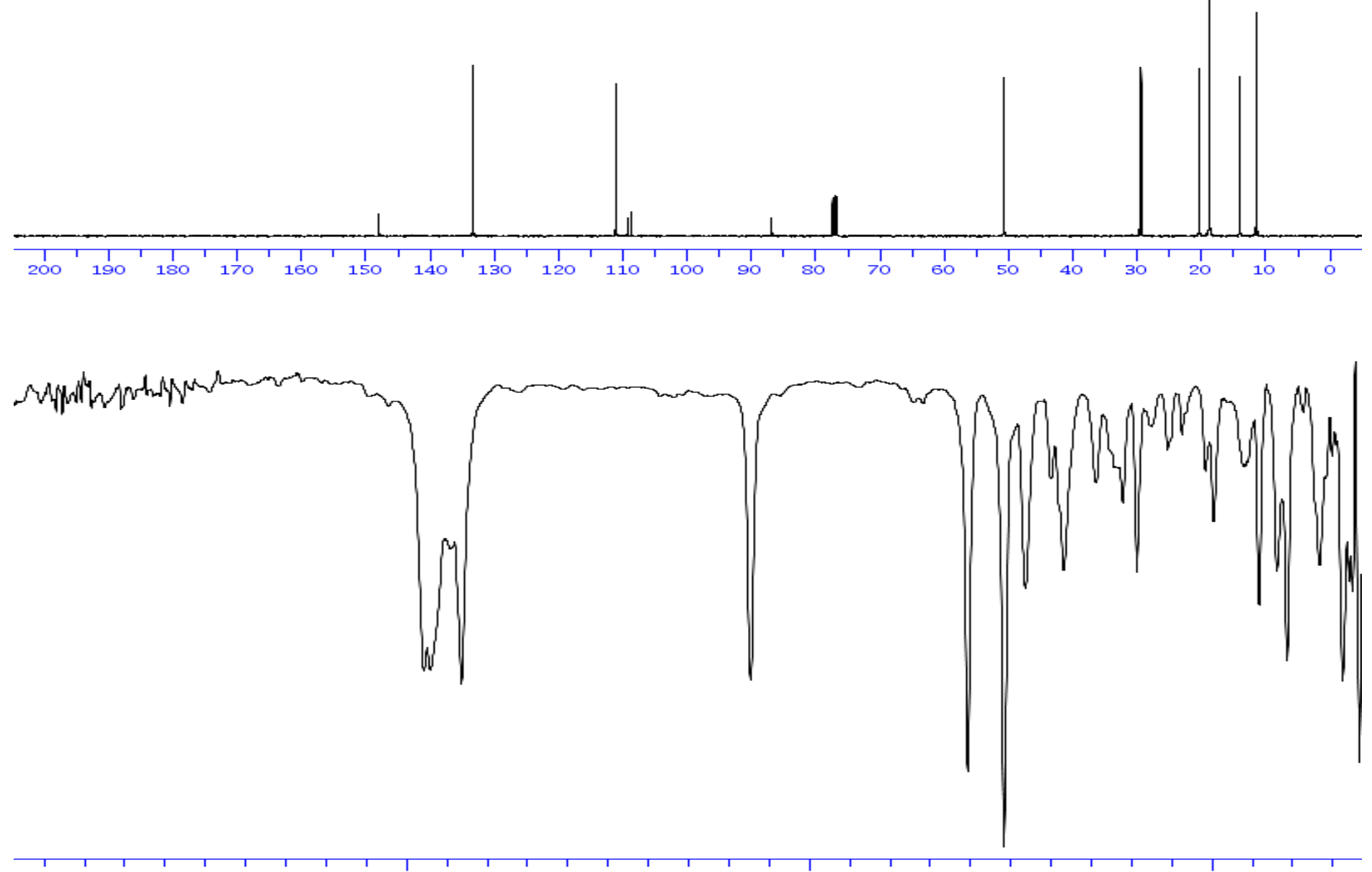
solvent: $<\mathrm{CDCI} 3\rangle$

Frequency $400.13 \mathrm{MHz}$
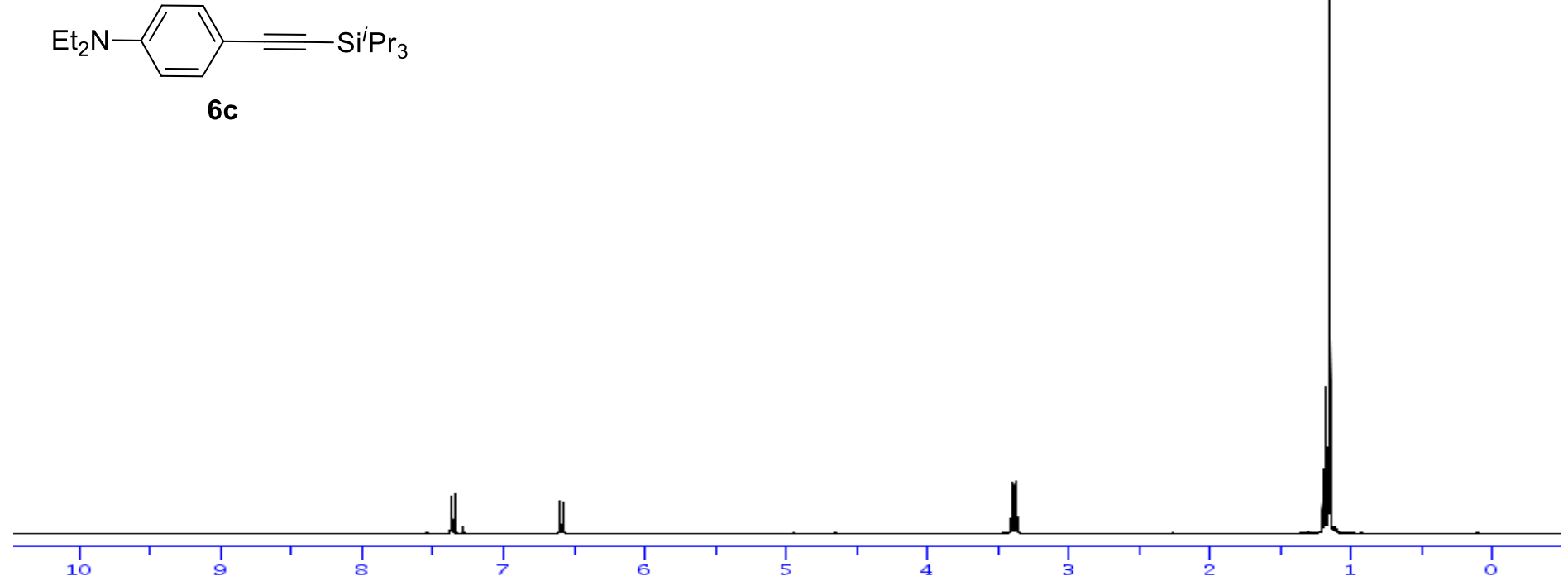

solvent: $<\mathrm{CDCI} 3\rangle$

Frequencr.100.612769MHz

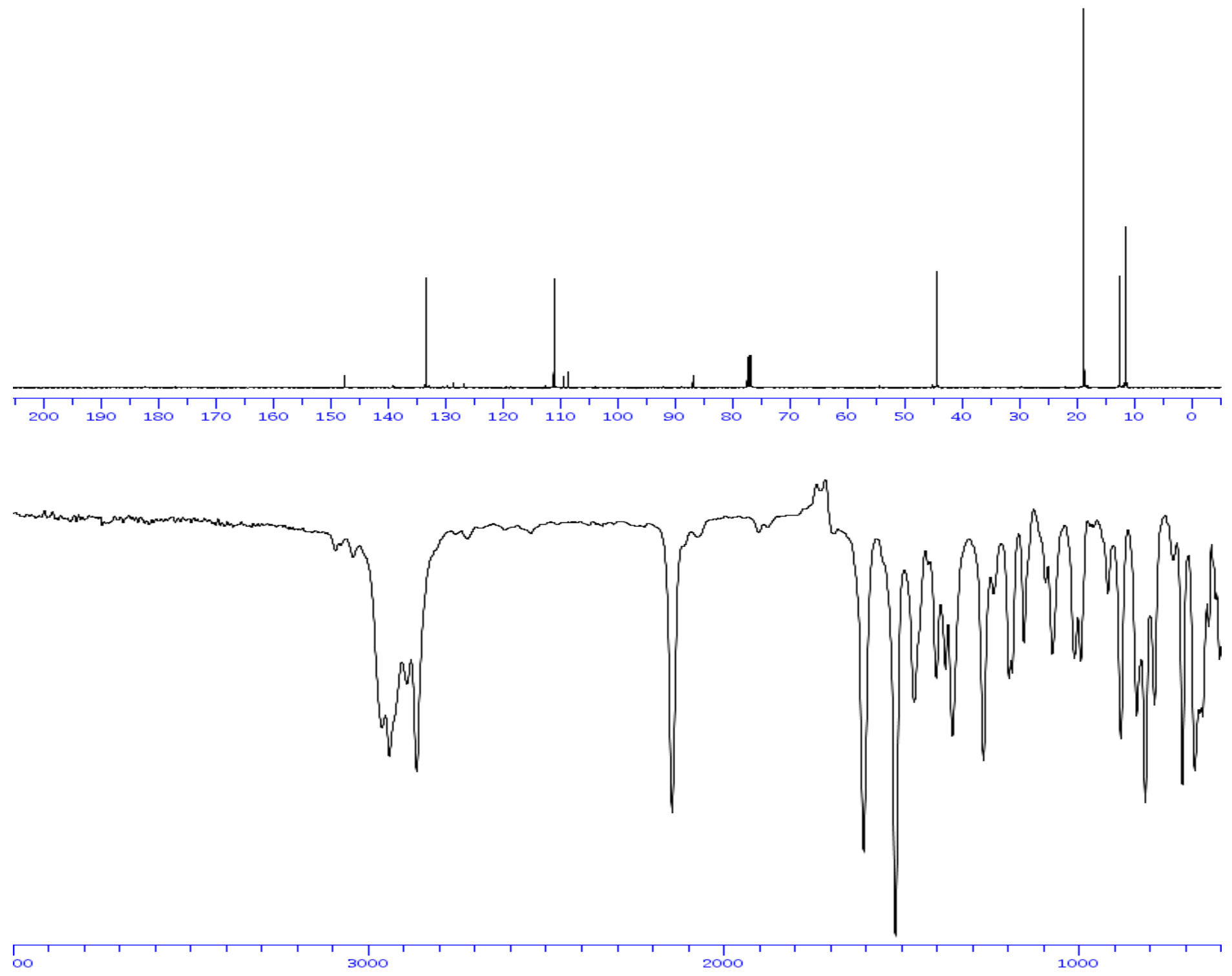


solvent: $<\mathrm{CDCI} 3\rangle$

Frequency $400.13 \mathrm{MHz}$

$\mathrm{Me}_{2} \mathrm{~N}=\mathrm{Si}_{6 \mathrm{~d}} \mathrm{Pr}_{3}$

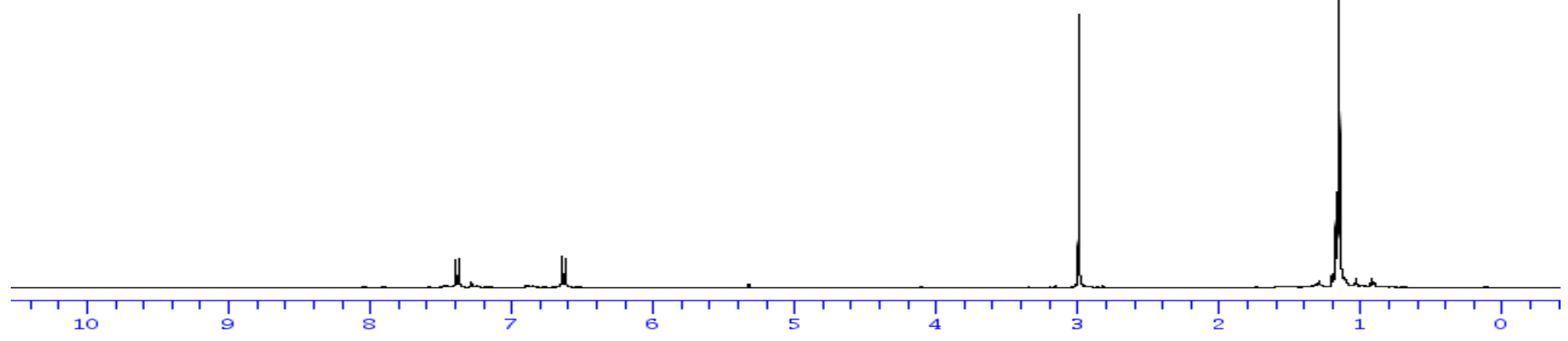

Solvent: $<C D C I 3>$
Frequency. $100.612769 \mathrm{MHz}$
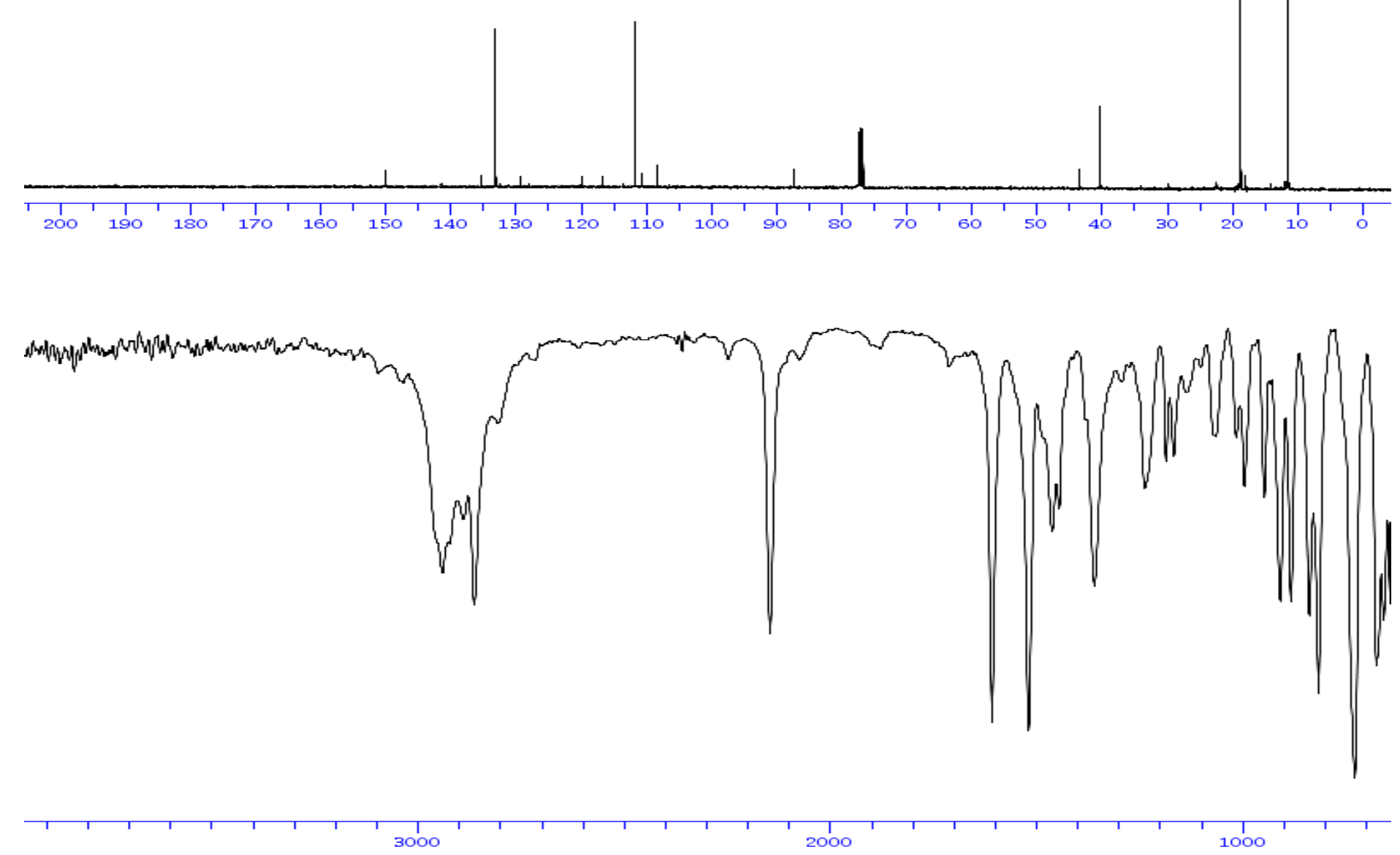
solvent: $<C D C I 3>$

Frequency.400.13 $\mathrm{MHz}$

$\mathrm{Me}_{2} \mathrm{~N}=\mathrm{Si}^{\mathrm{P}} \mathrm{Pr}_{3}$

24

${ }^{i} \mathrm{Pr}_{3} \mathrm{Si}$
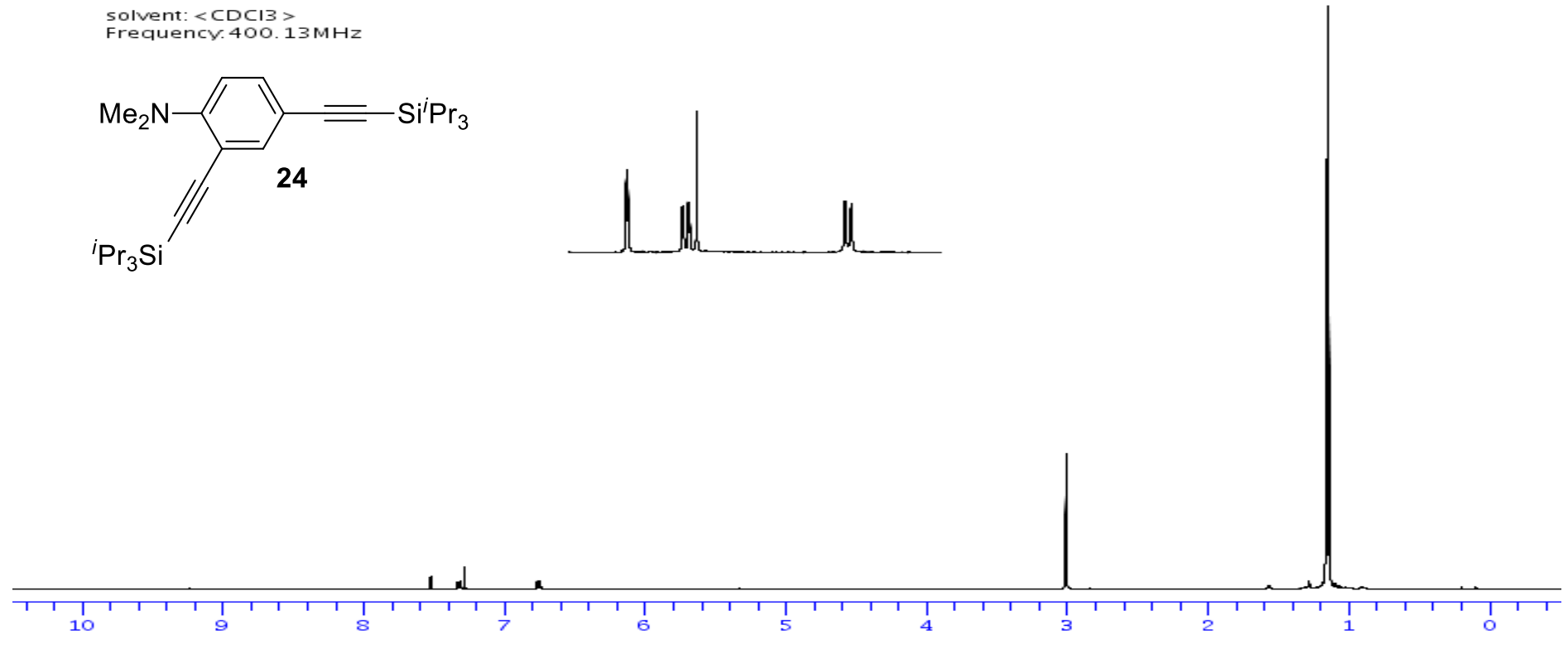

Solvent: $<C D C I 3>$
Frequency. $100.612769 \mathrm{MHz}$

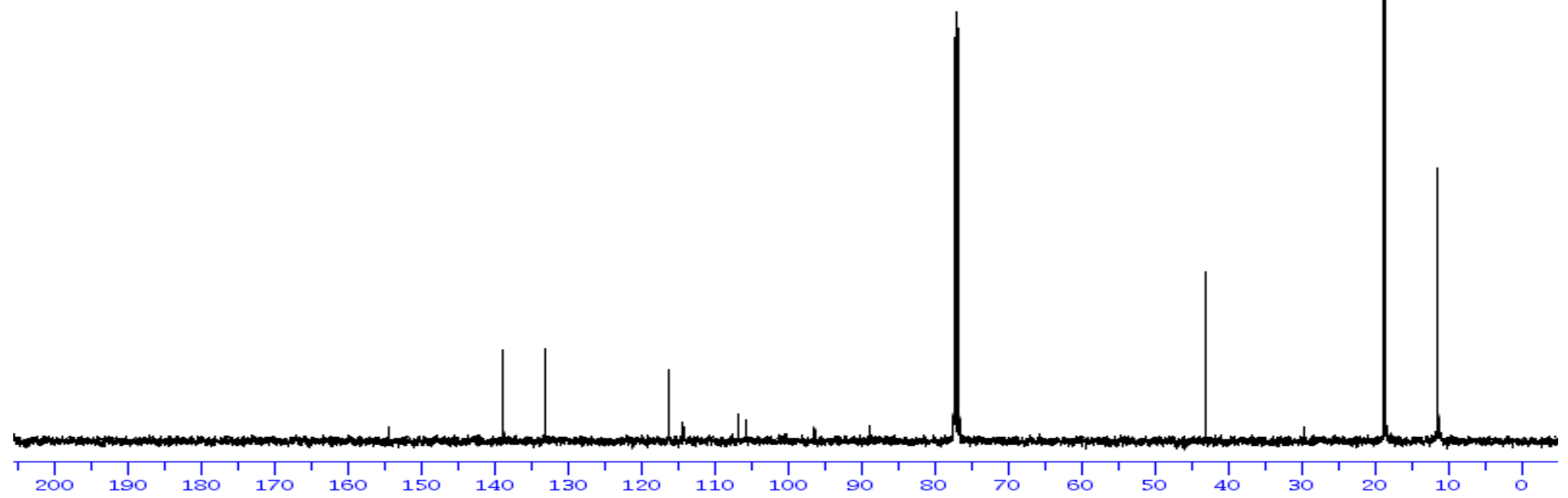

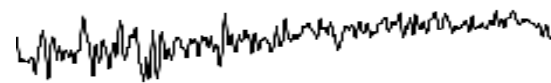
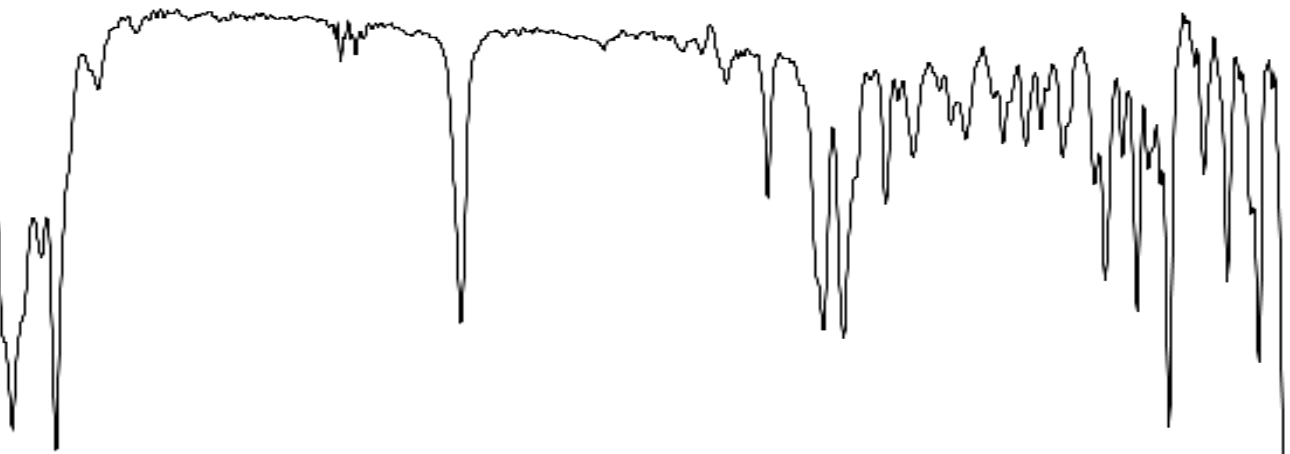
solvent: $<C D C I 3>$

Frequency.400.13 $\mathrm{MHz}$
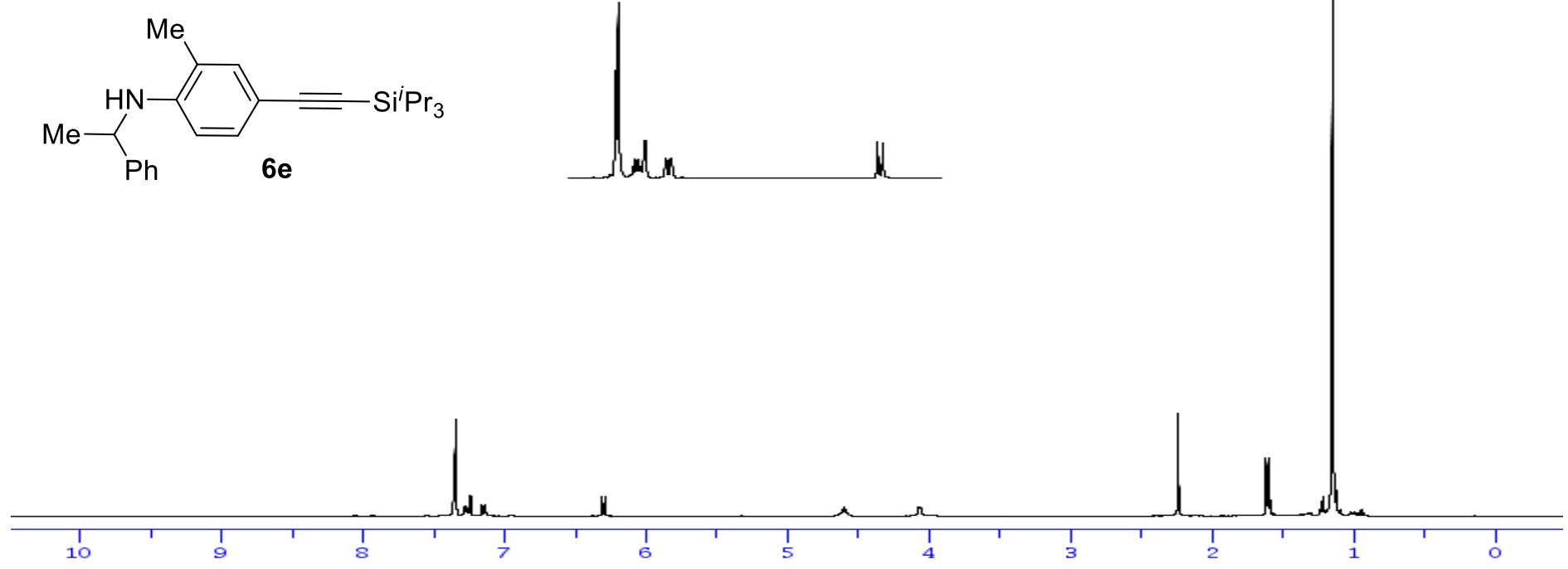

solvent: $<\mathrm{CDCI} 3>$

Frequency. $100.612769 \mathrm{MHz}$
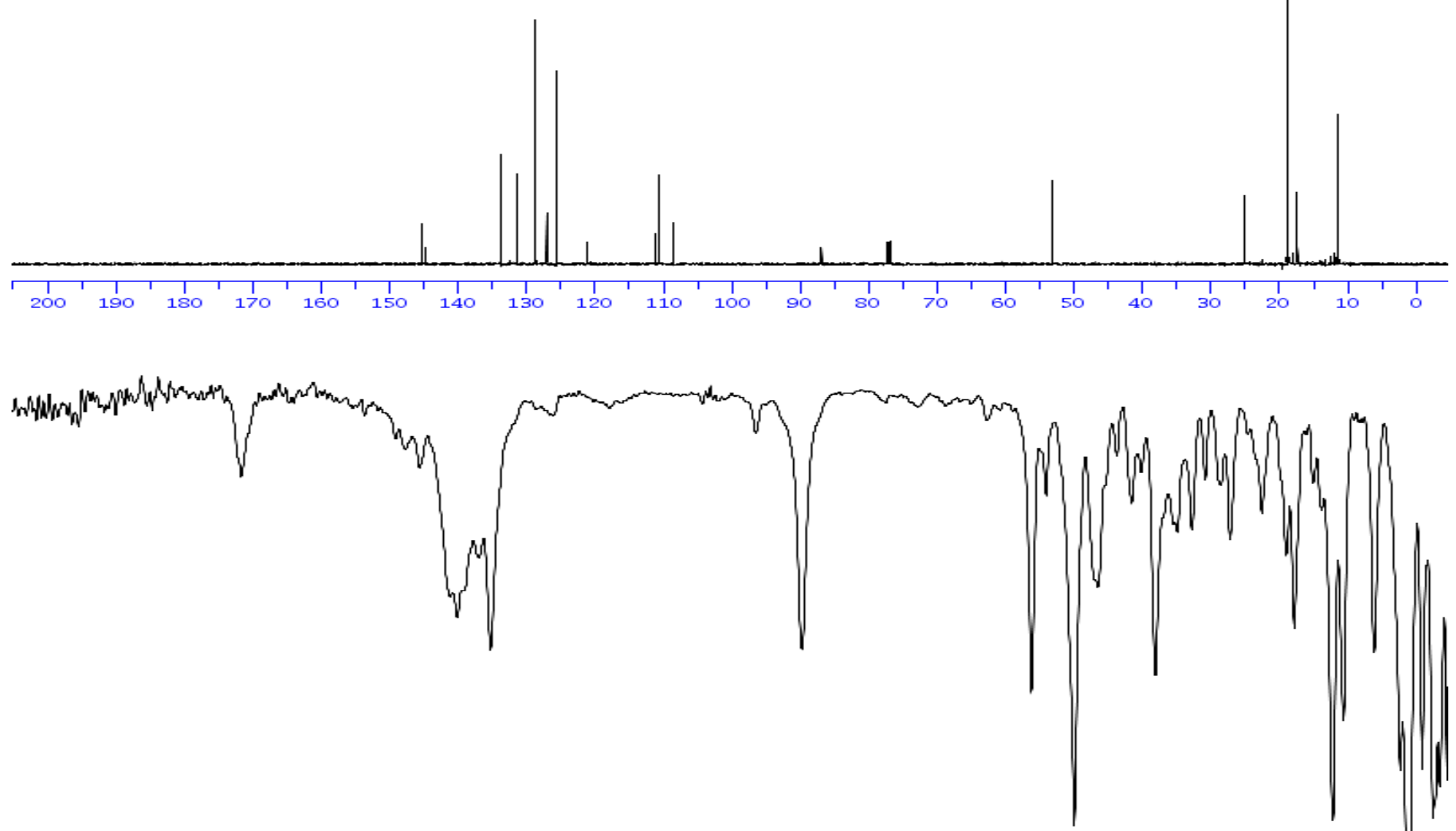
solvent: $<C D C I 3>$

Frequency.400.13 $\mathrm{MHz}$<smiles>CC(Nc1ccccc1)c1ccccc1</smiles>
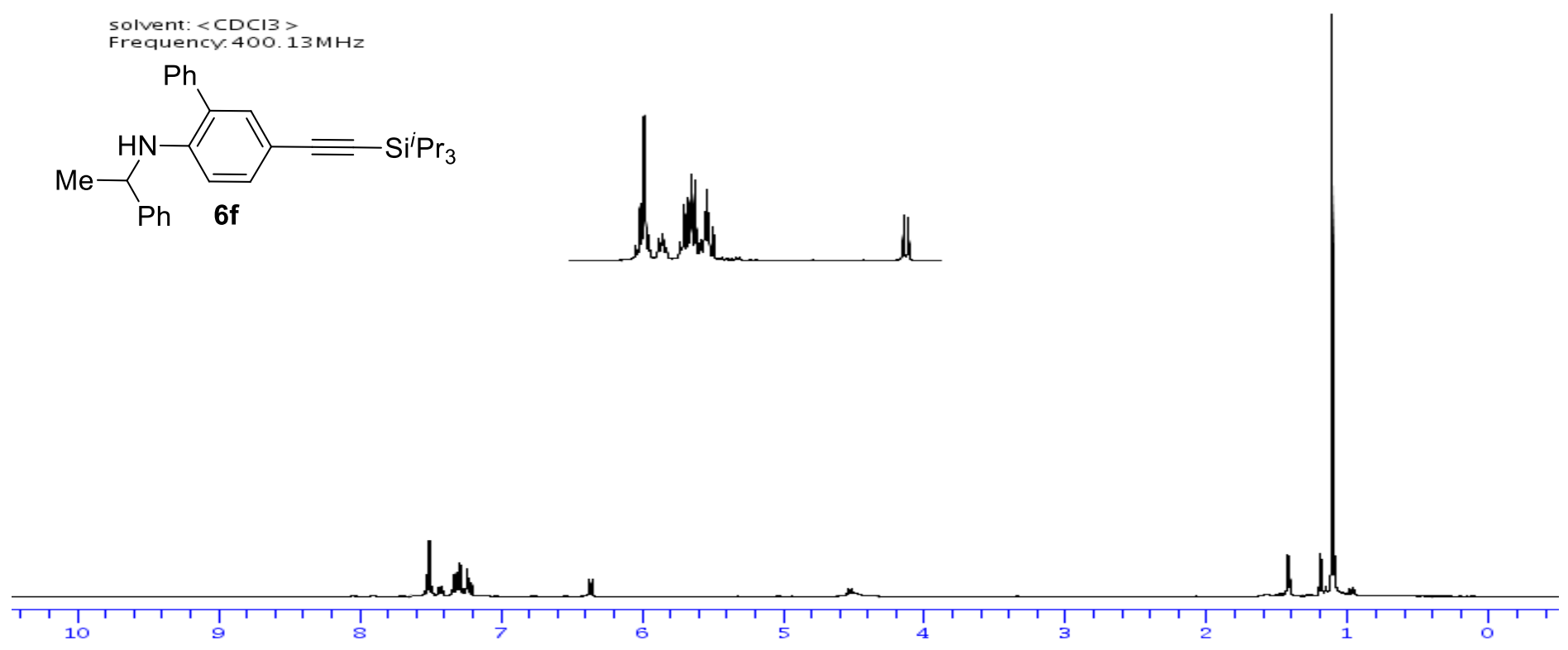

solvent: $<C D C I 3>$
Frequency. $100.612769 \mathrm{MHz}$

quency. $100.612769 \mathrm{MHz}$
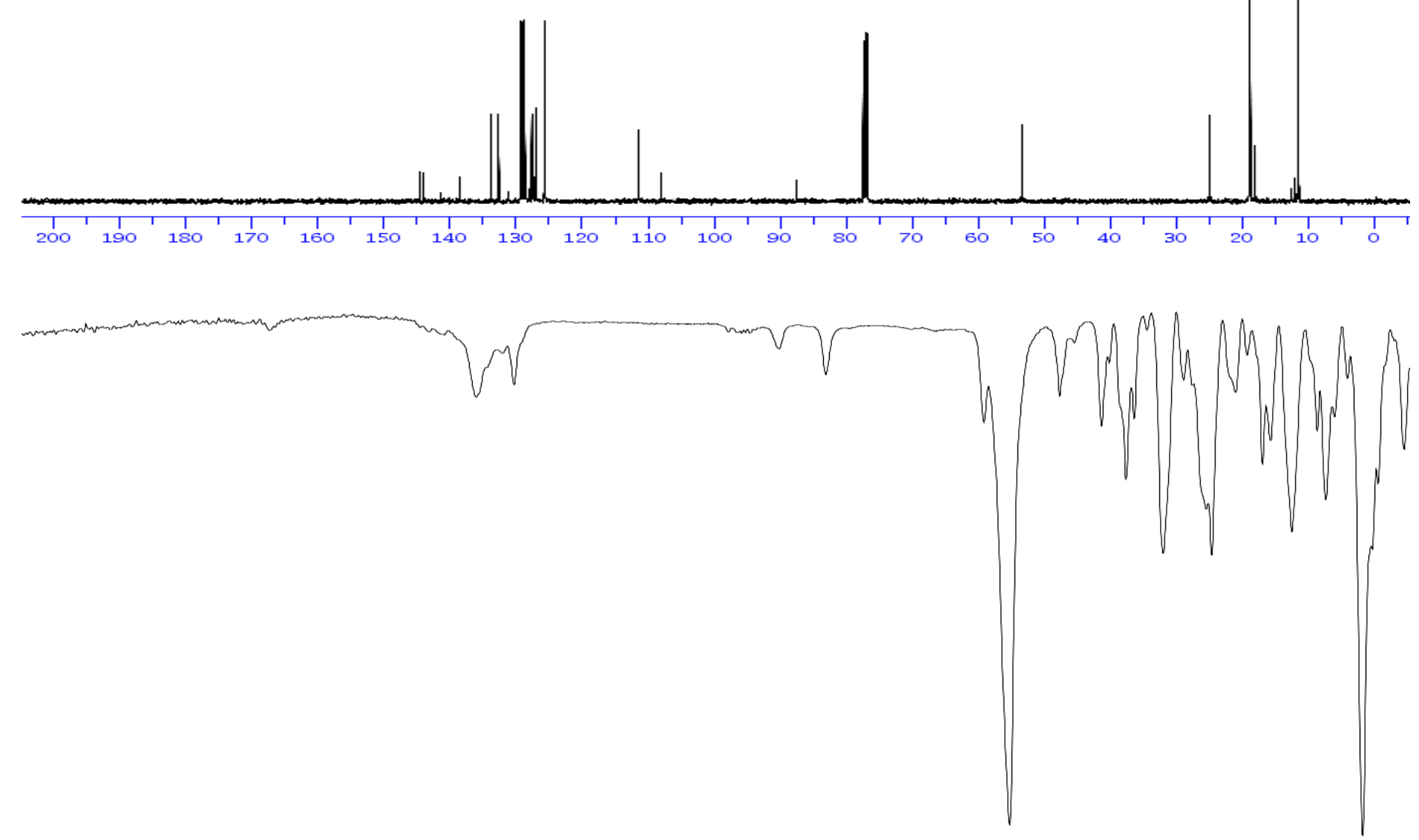
<smiles>COc1cc(C#C[SiH2][Na])ccc1NC(C)c1ccccc1</smiles>
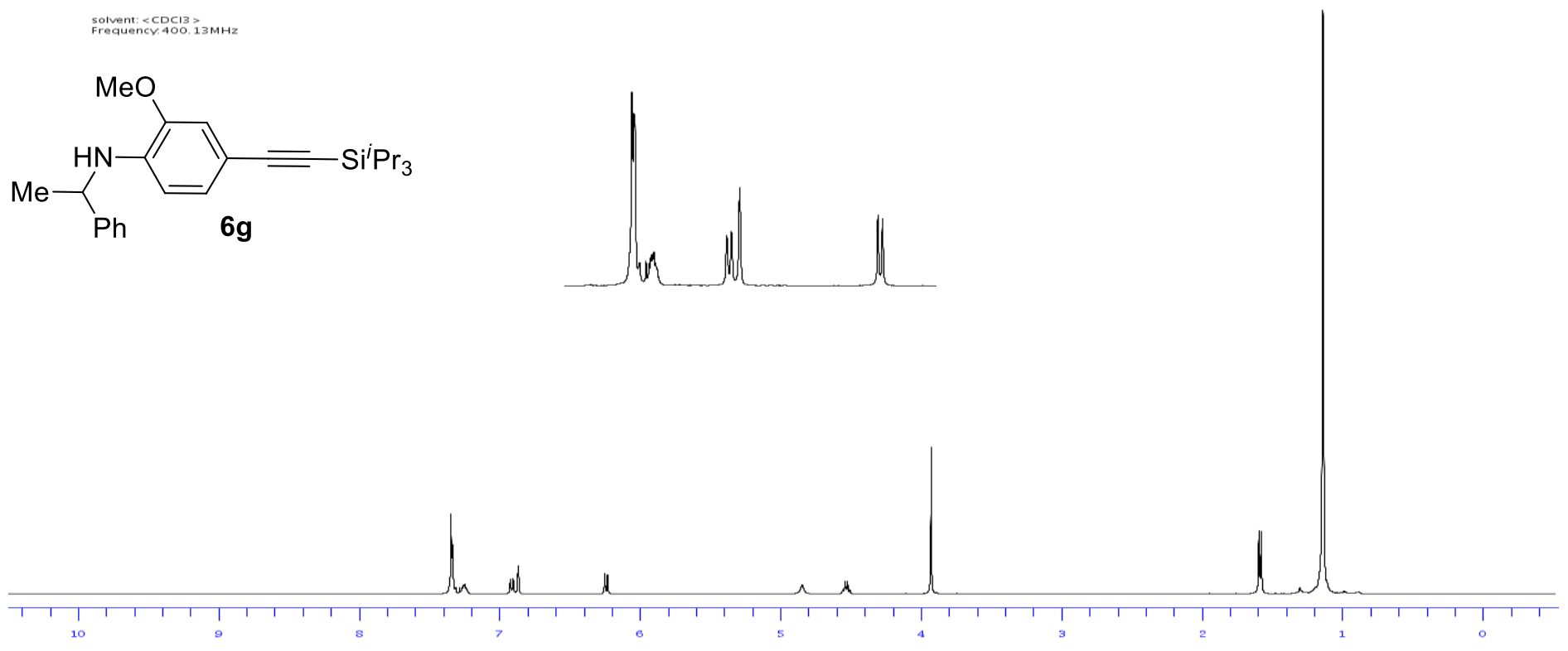

Solvent: $<C D C I 3>$
Frequency. $100.612769 \mathrm{MHz}$
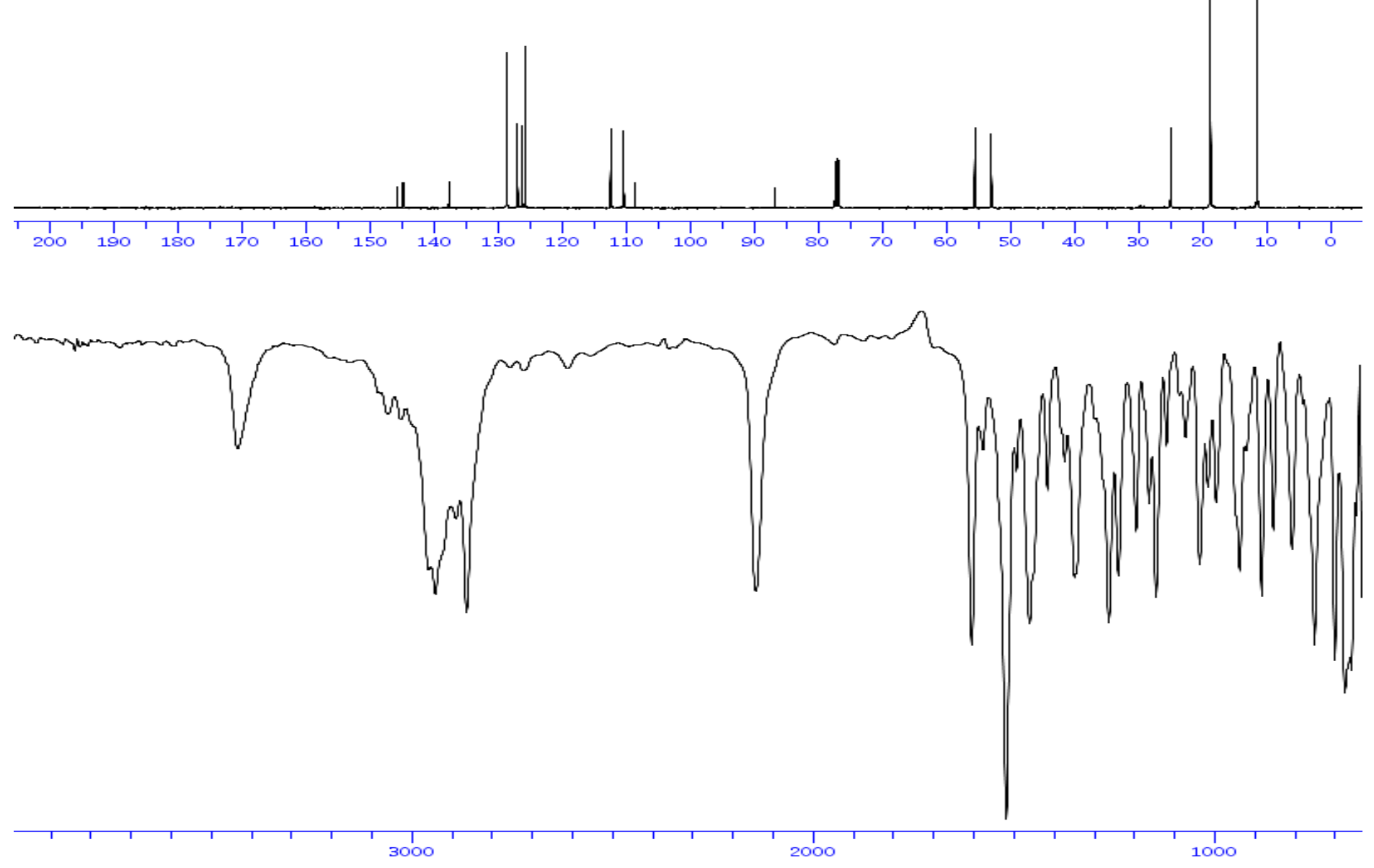

28 
solvent: <CDCI3 >

Frequency. 400.13 $\mathrm{MHz}$

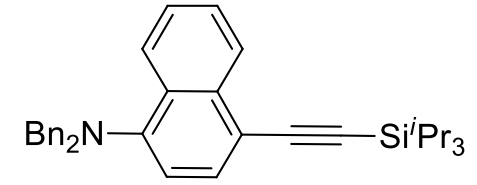

$6 h$

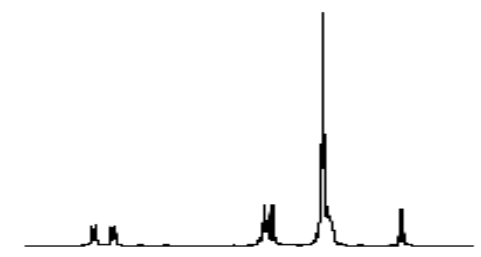

1

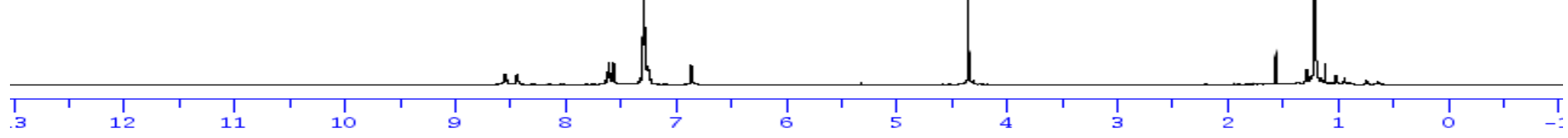

Solvent: $<C D C I 3>$
Frequency. $100.612769 \mathrm{MHz}$
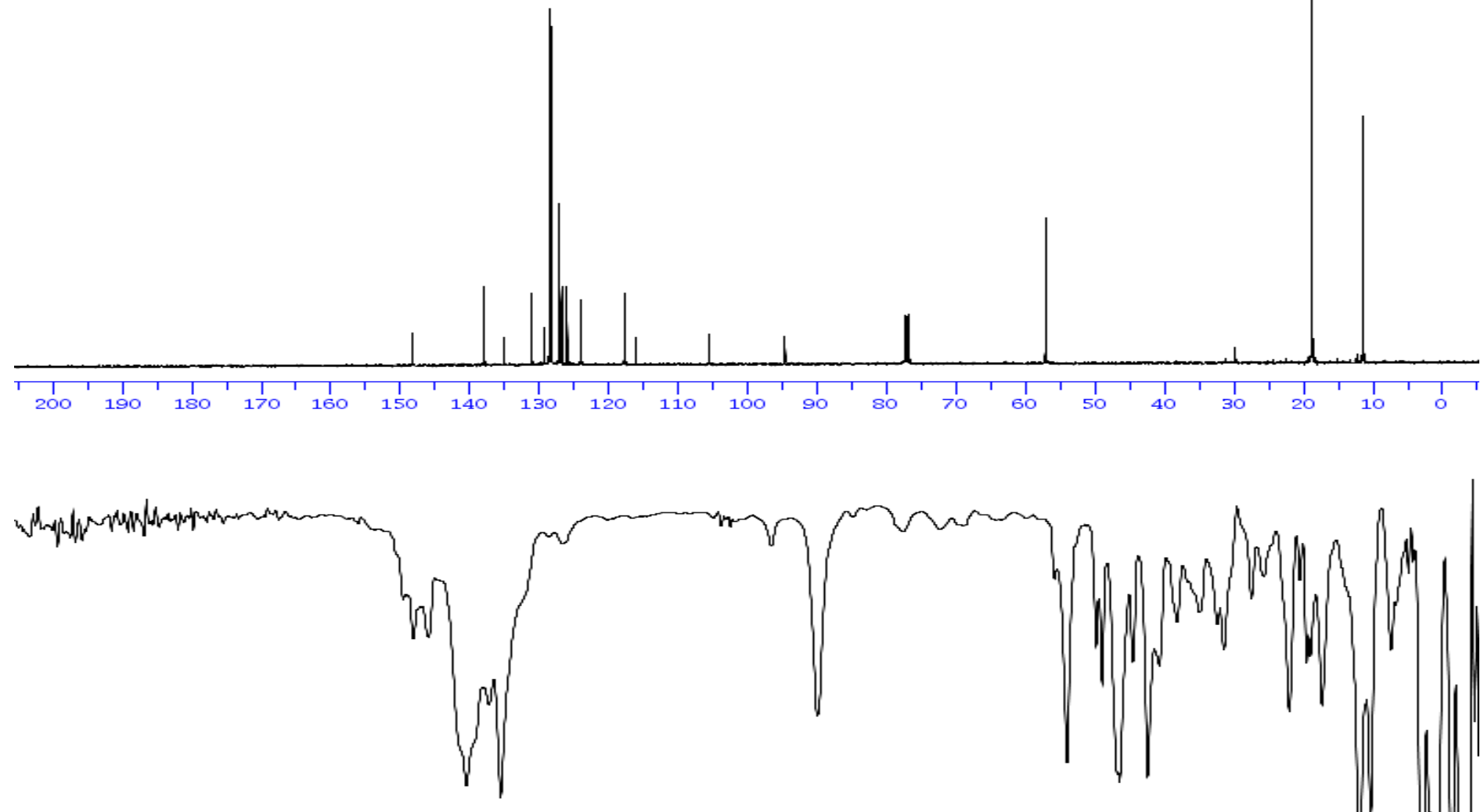
solvent: <CDCI3 $>$

Frequency.400.13 $\mathrm{MHz}$
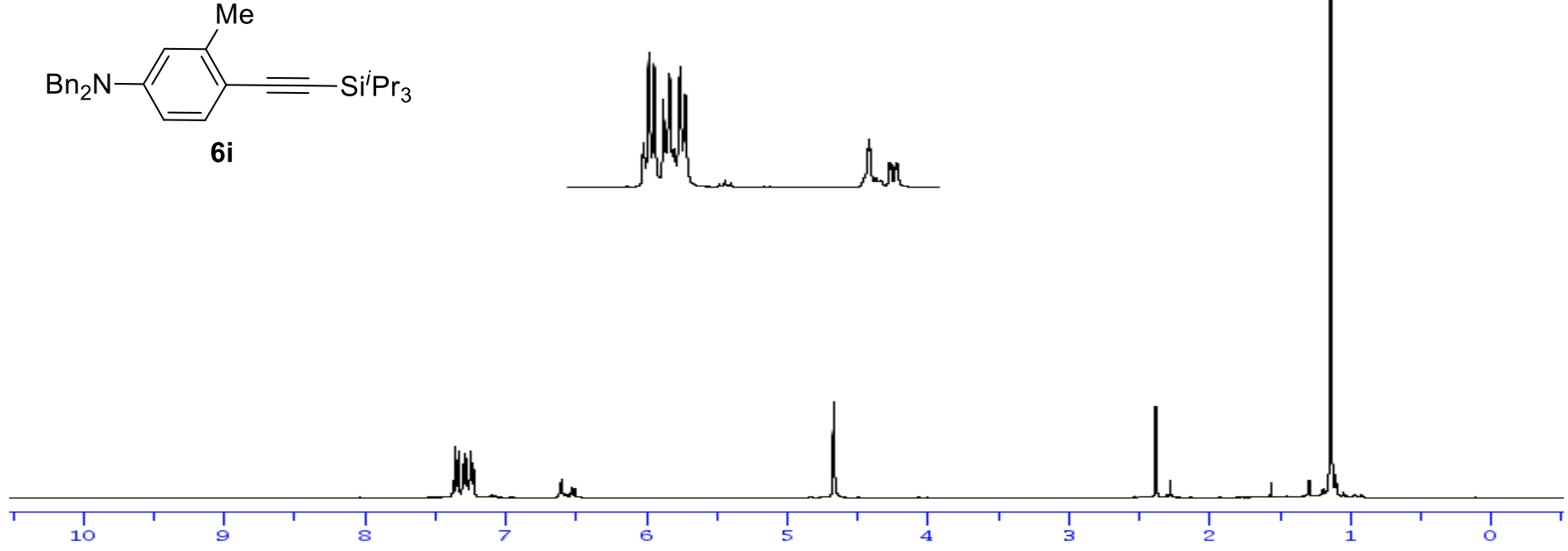

Solvent: $<C D C I 3>$

. $12769 \mathrm{MHz}$
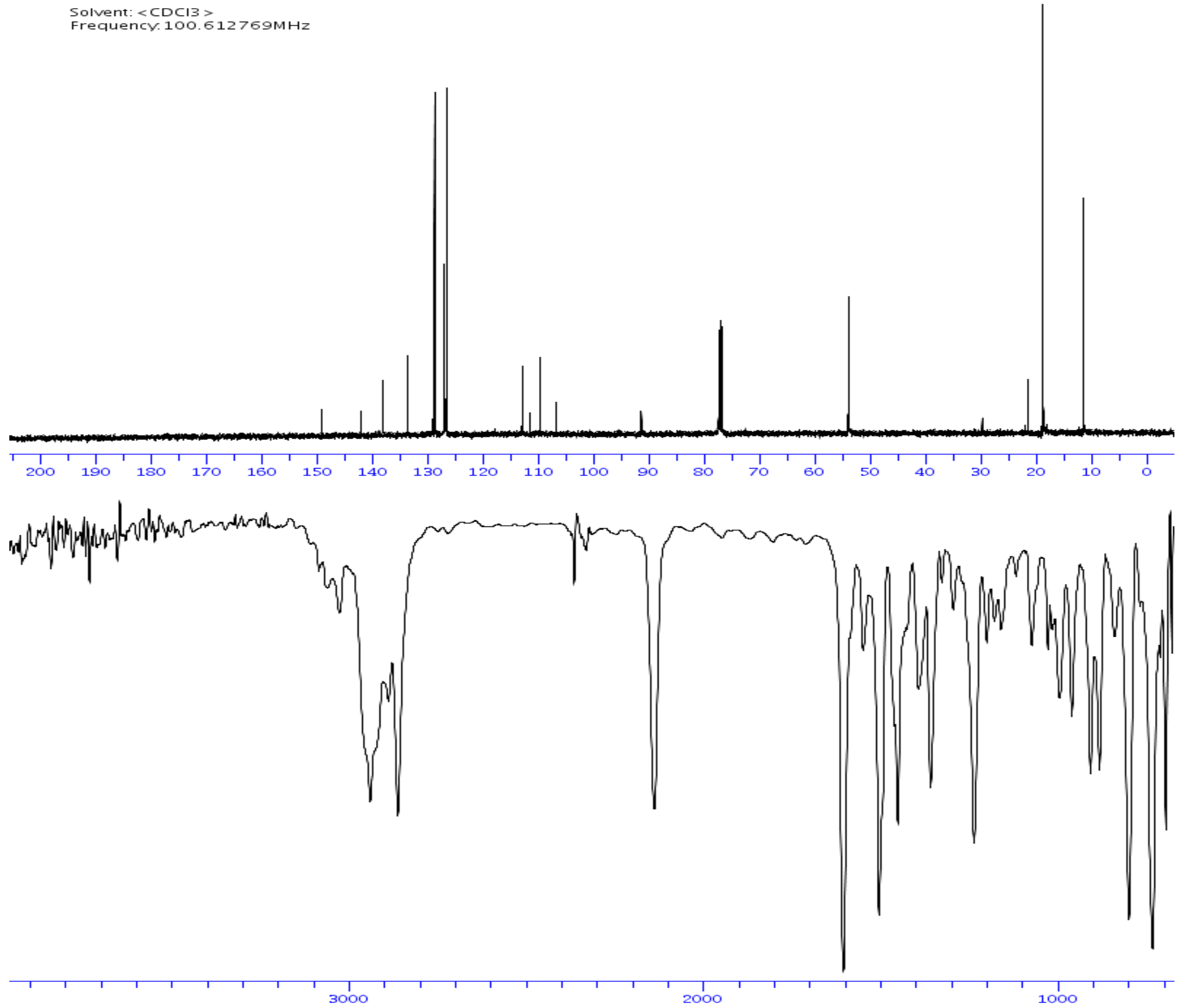
<smiles>COc1cc(N(Cc2ccccc2)CC(C)(C)C)ccc1C#C[SiH2]</smiles>

6j
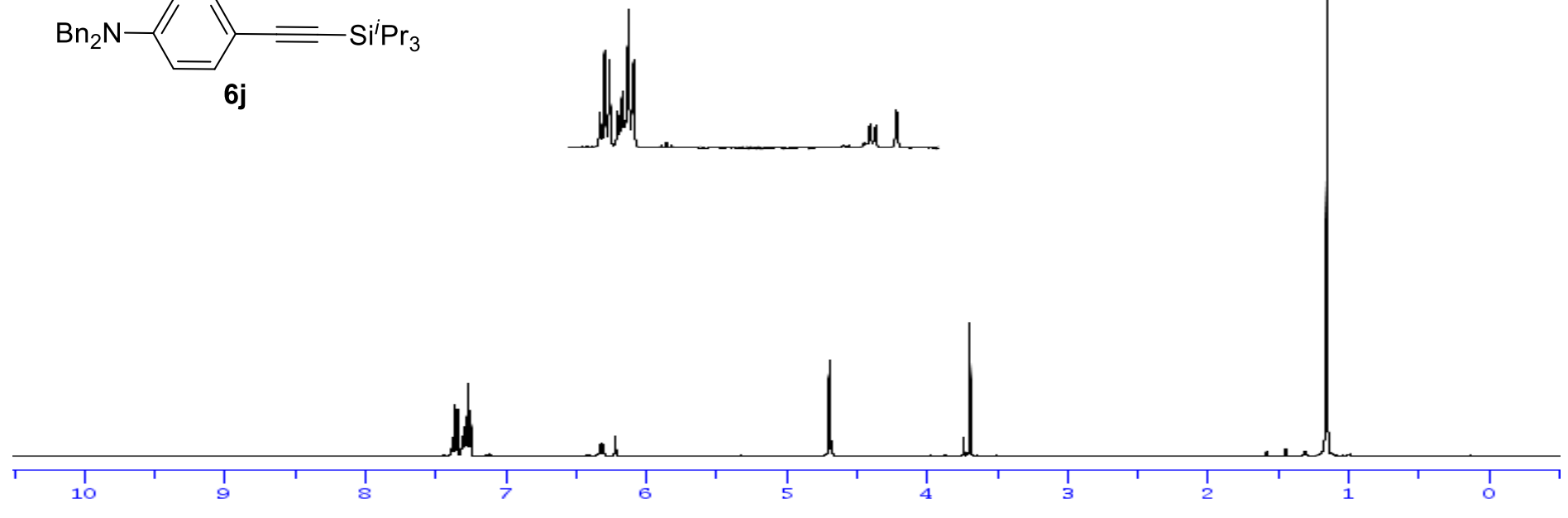

solvent: $<C D C I 3>$

Frequency. $100.612769 \mathrm{MHz}$
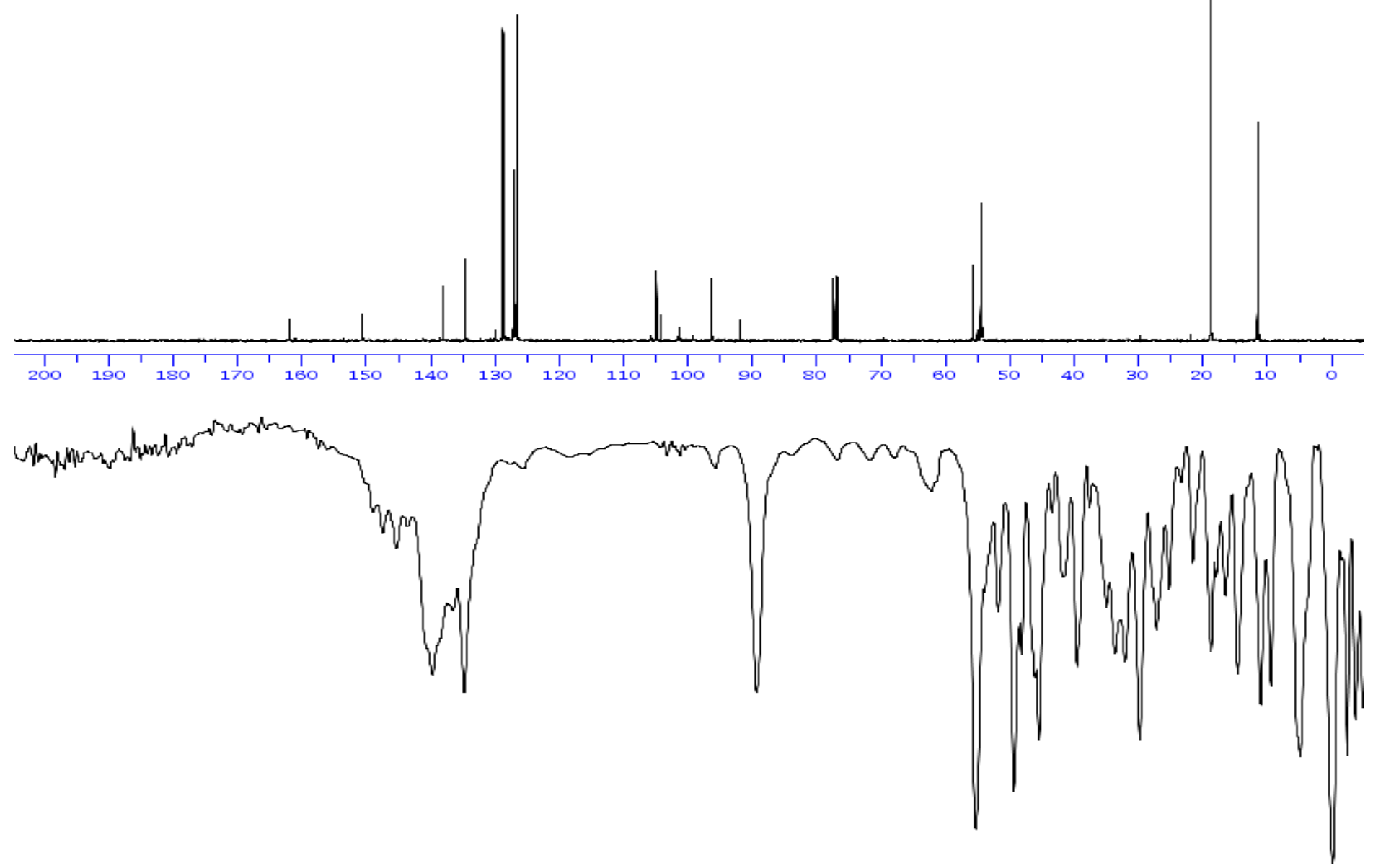


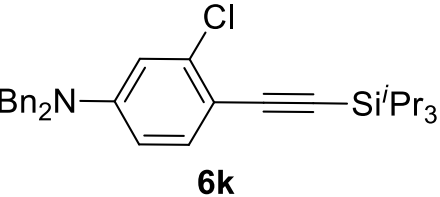

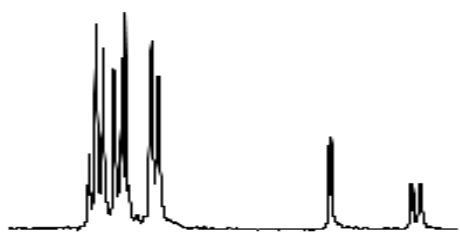

Wh 1 .

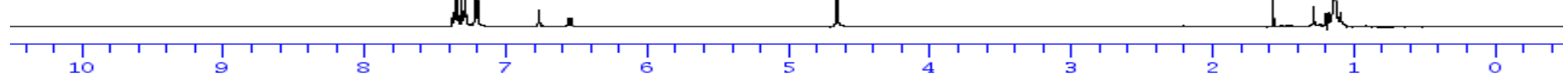

solvent: $<C D C I 3>$
Frequency. $100.612769 \mathrm{MHz}$

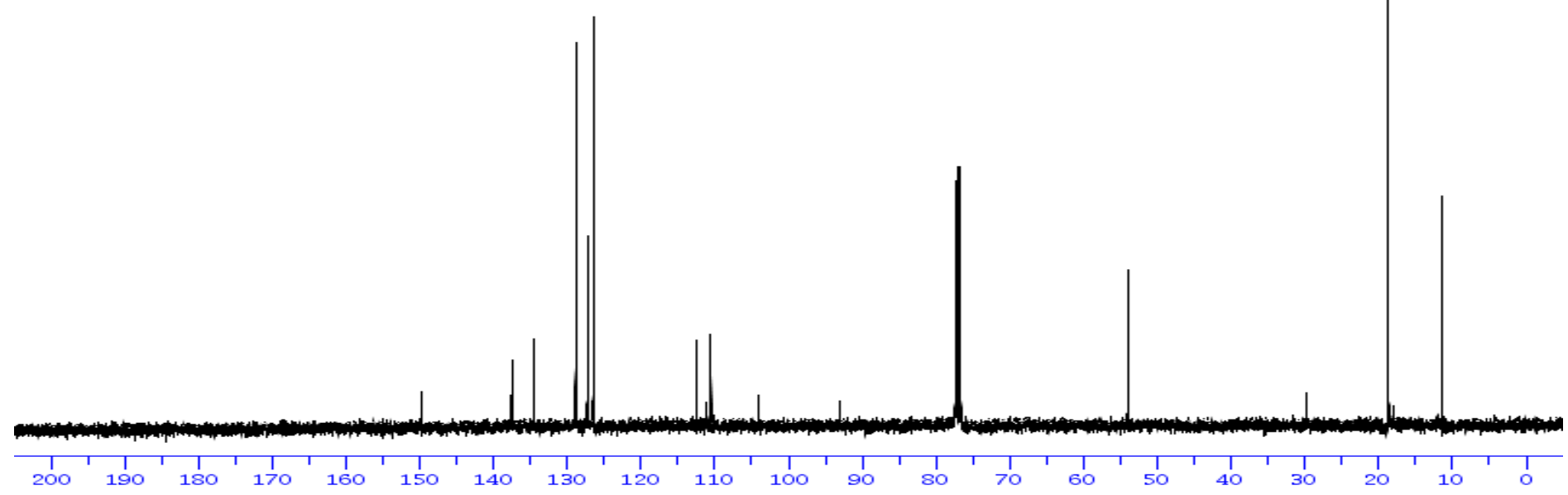

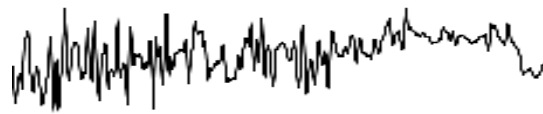
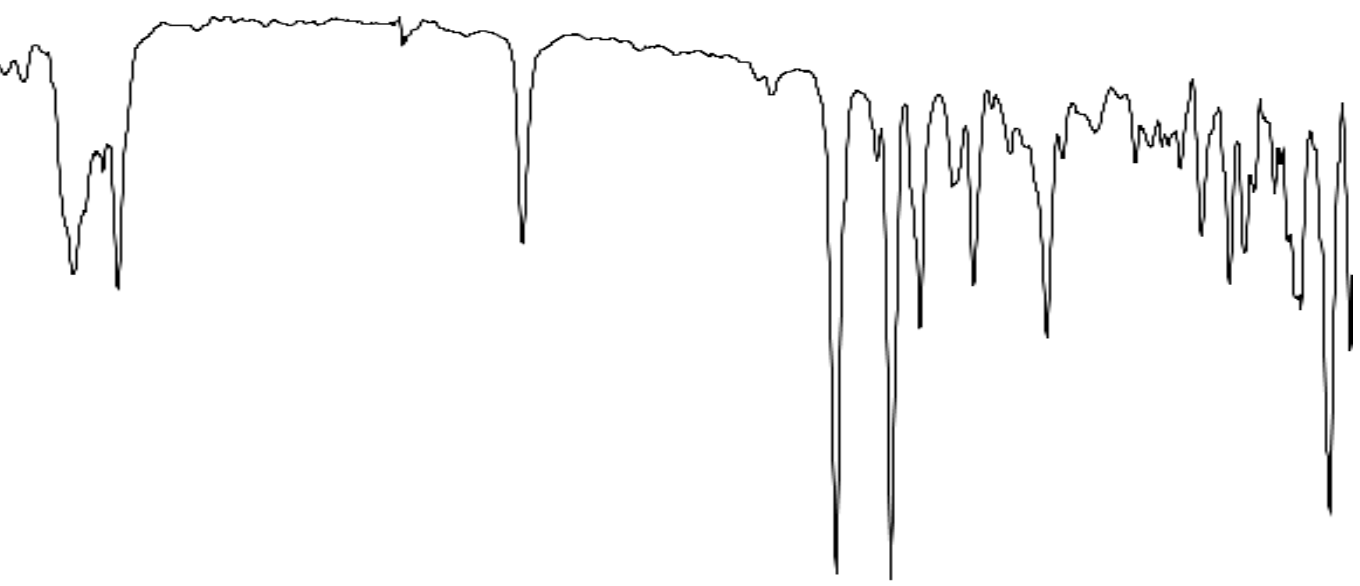
solvent: $\angle \mathrm{CDCI} 3\rangle$

Frequency.400.13 $\mathrm{MHz}$

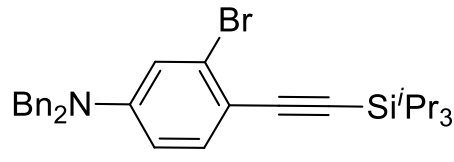

6I
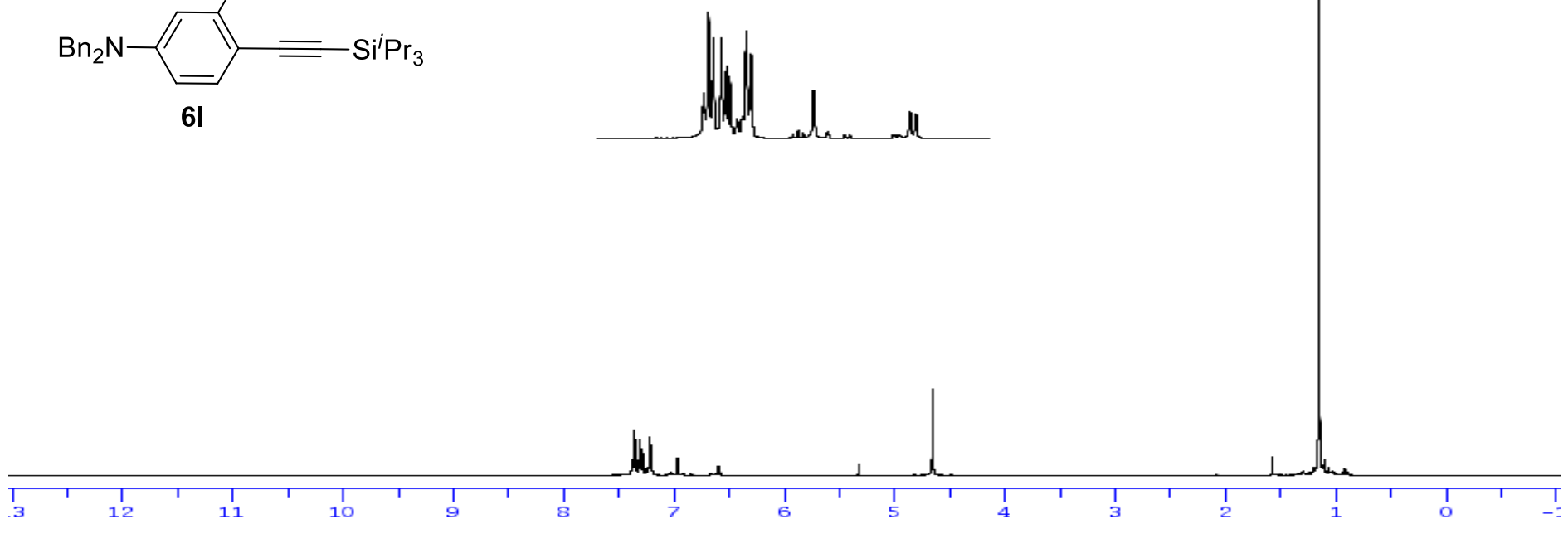

solvent: $<\mathrm{CDCI} 3$

equency. $100.612769 \mathrm{MHz}$
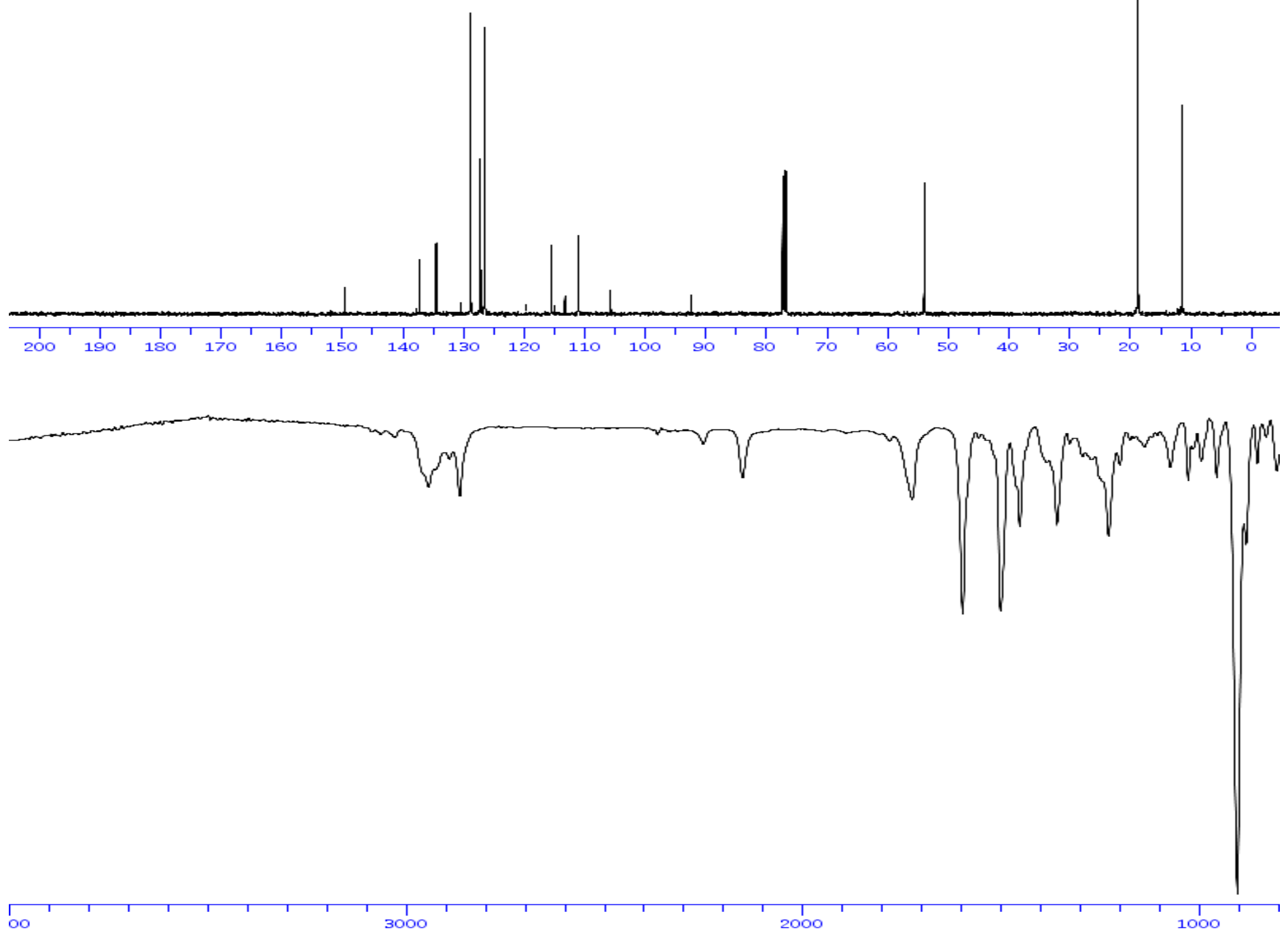
solvent: $<\mathrm{CDCI} 3$

Frequency. $400.13 \mathrm{MHz}$

$\mathrm{Et}_{2} \mathrm{~N}=\mathrm{Si}^{\mathrm{i}} \mathrm{Pr}_{3}$

$6 m$
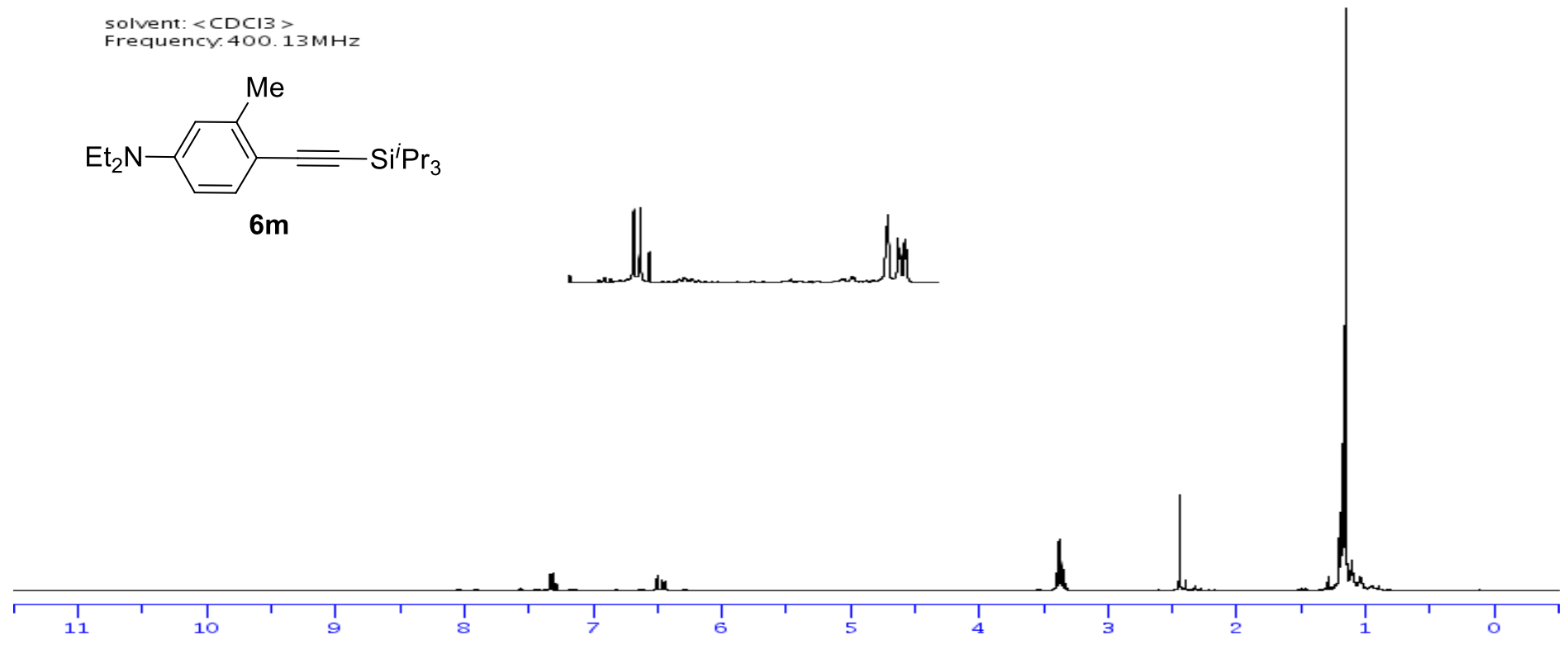

solvent: $<\mathrm{CDCI} 3$

Frequencr.100.612769MHz
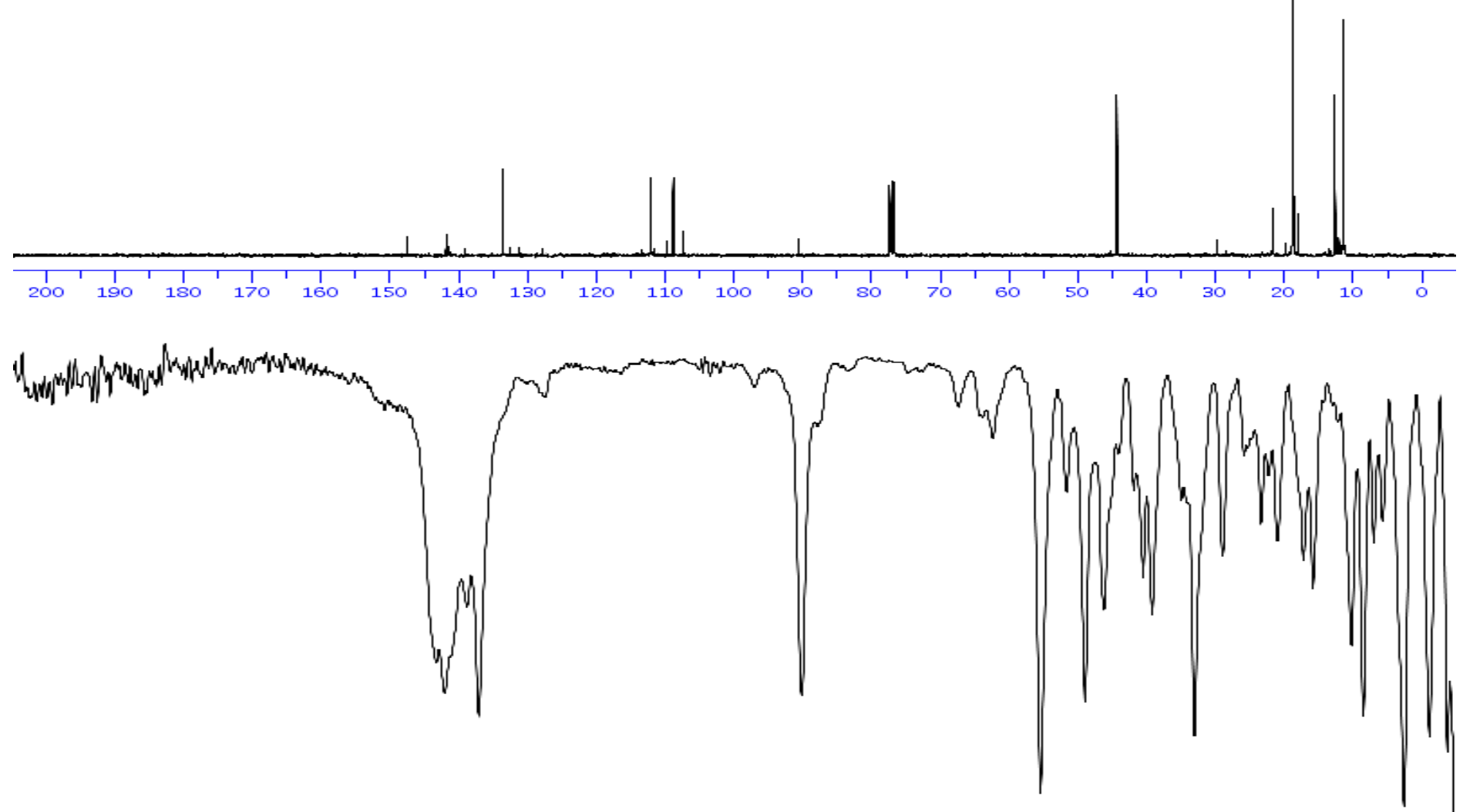
Solvent: $<\mathrm{CDCl} 3>$

Frequency. $400.13 \mathrm{MHz}$
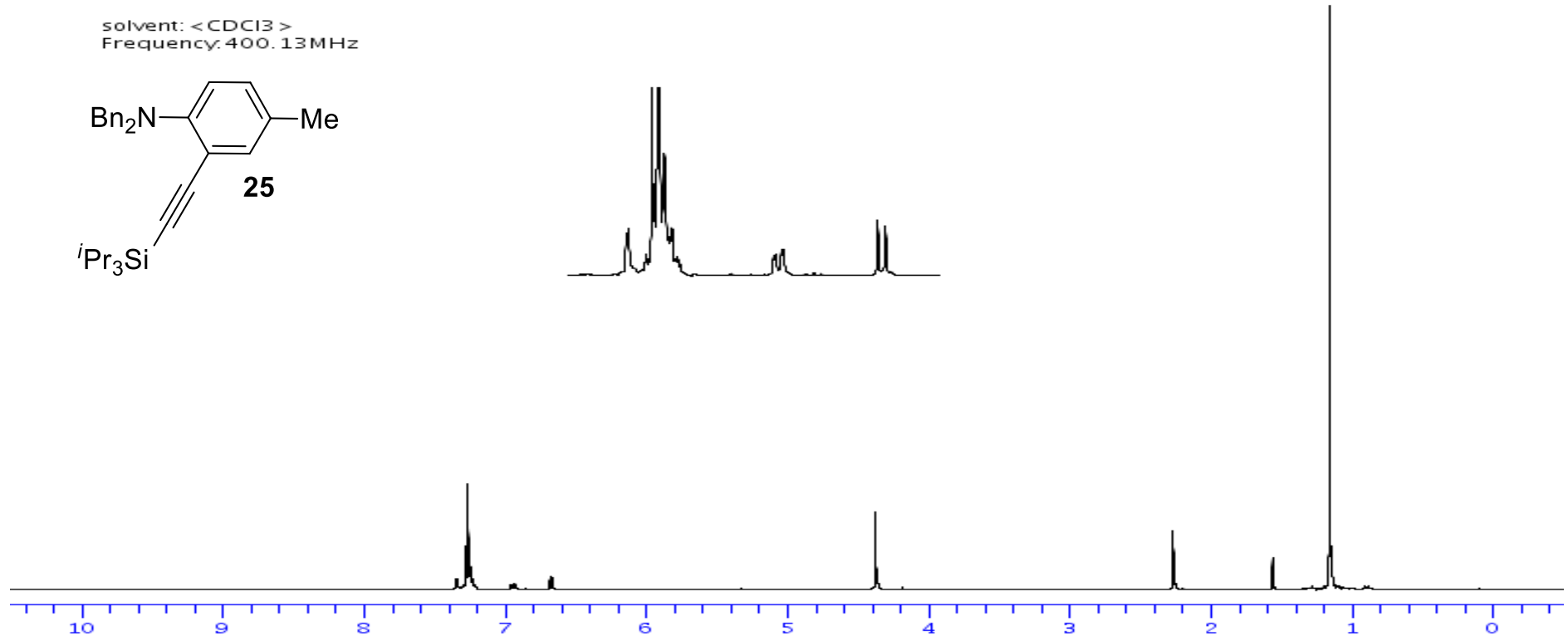

solvent: $<\mathrm{CDCI} 3>$
Frequency. $100.612769 \mathrm{MHz}$
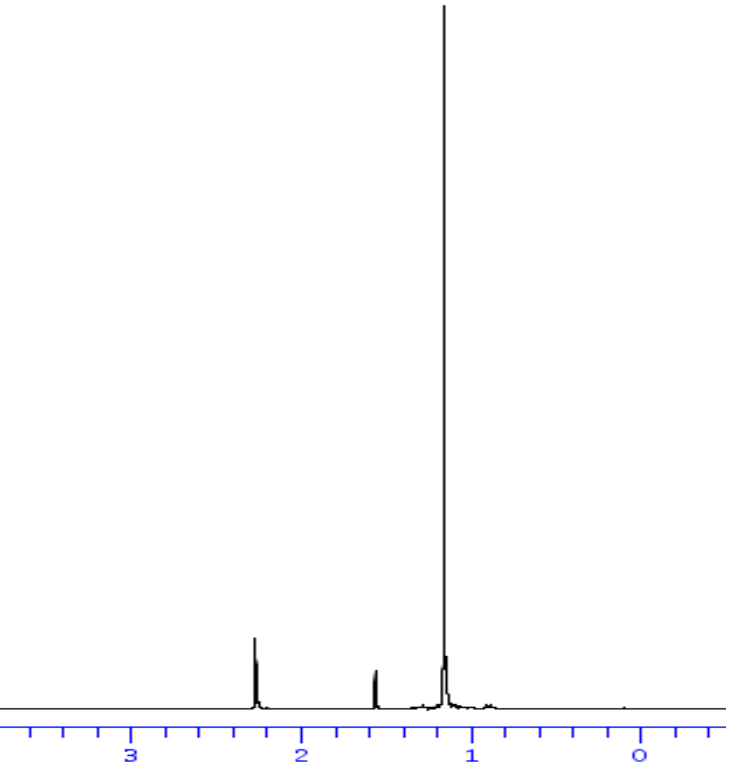

Five

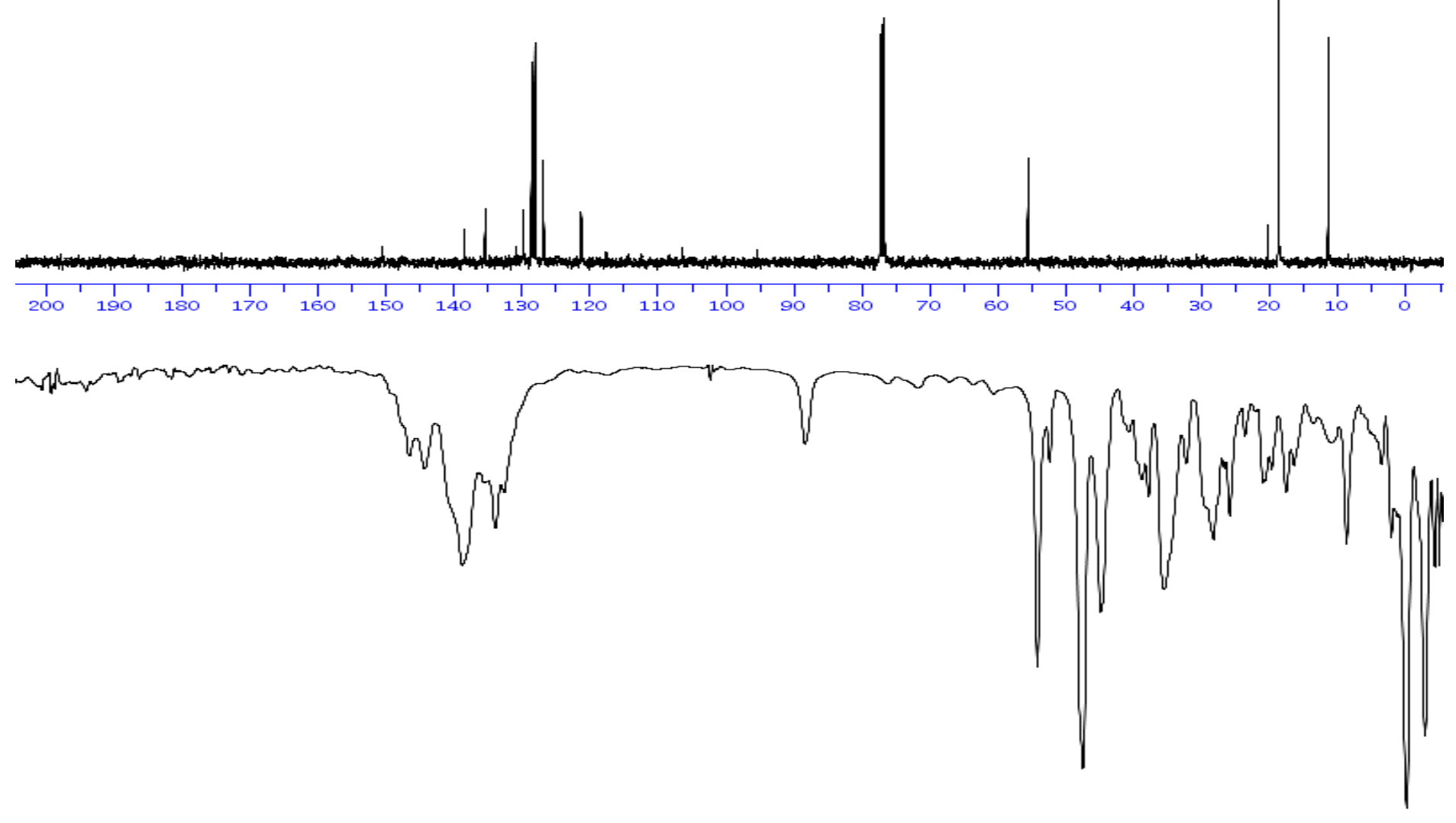


solvent: <CDCI3 >

(3)

$\mathrm{Me}$<smiles>Cc1ccc(N(C)CC#C[SiH2]C(C)C)cc1</smiles>

\section{al}

Solvent: $<$ CDCI3 $>$
Frequency.100.612769MHz
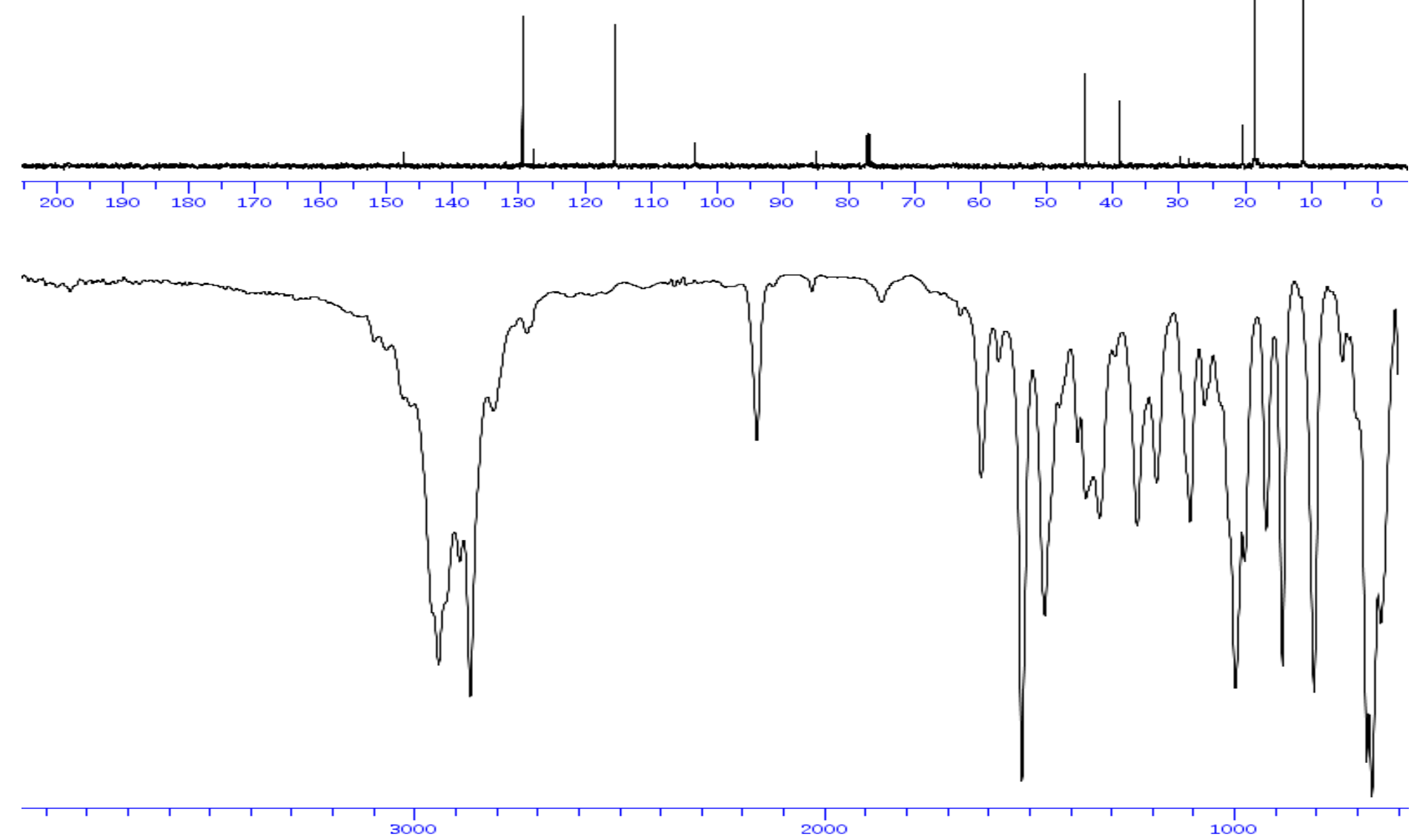
solvent: $<C D C I 3>$
Frequency. $400.13 \mathrm{MHz}$
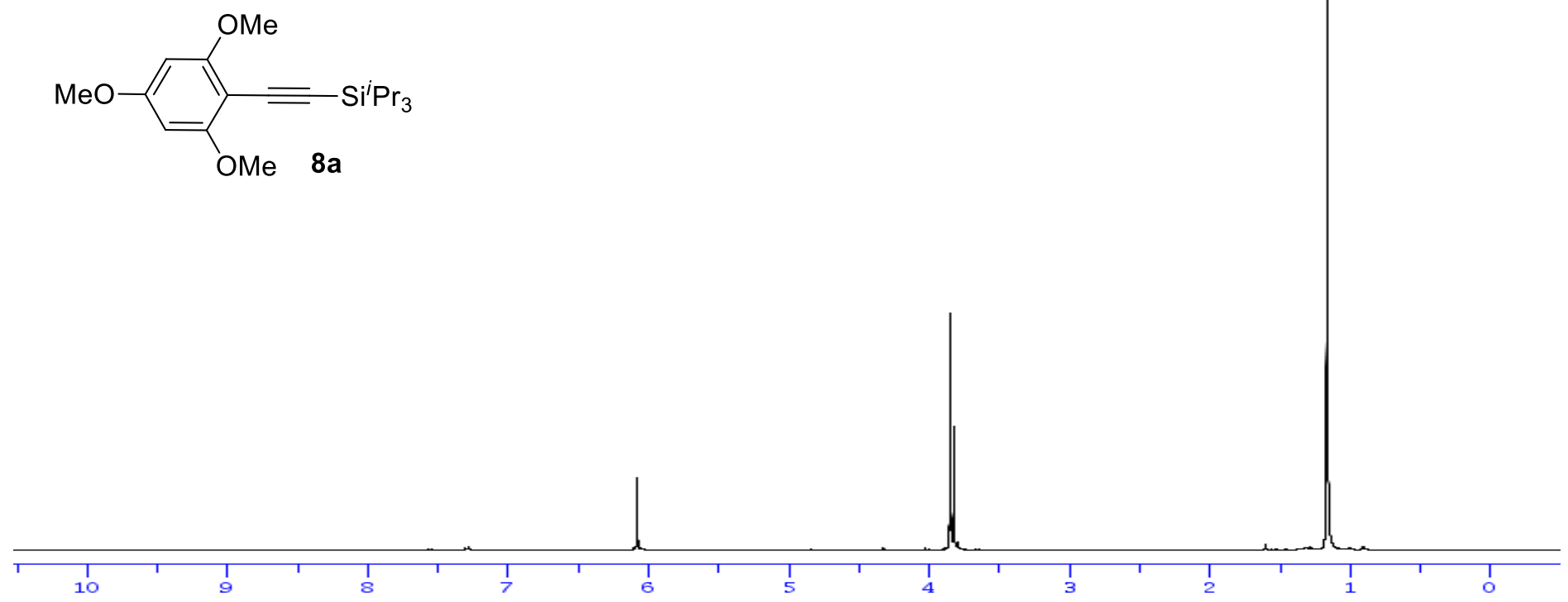

solvent: $<C D C I 3>$
Frequency. $100.612769 \mathrm{MHz}$
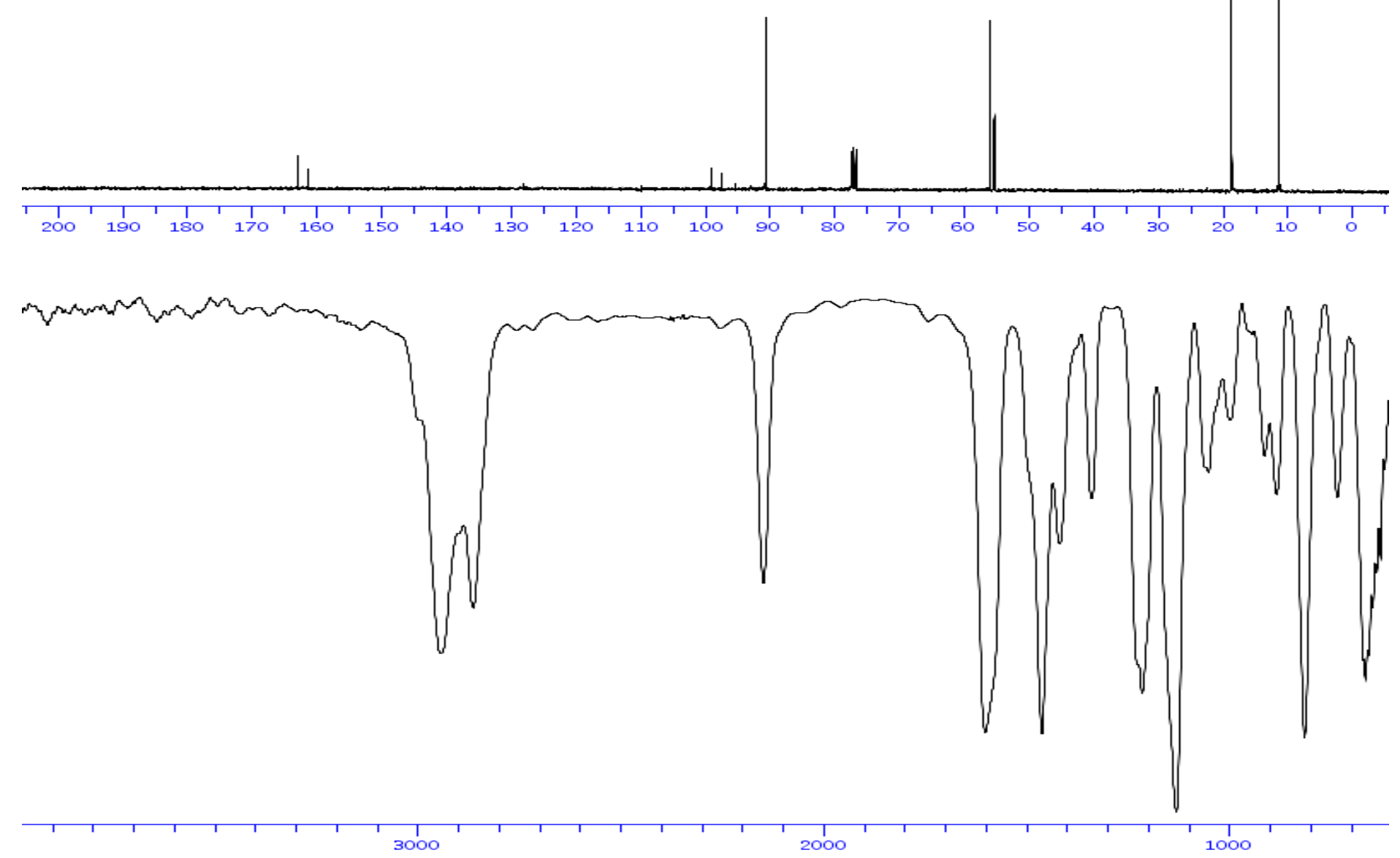
solvent: $\angle \mathrm{CDCI} 3>$

Frequencr $400.13 \mathrm{MHz}$
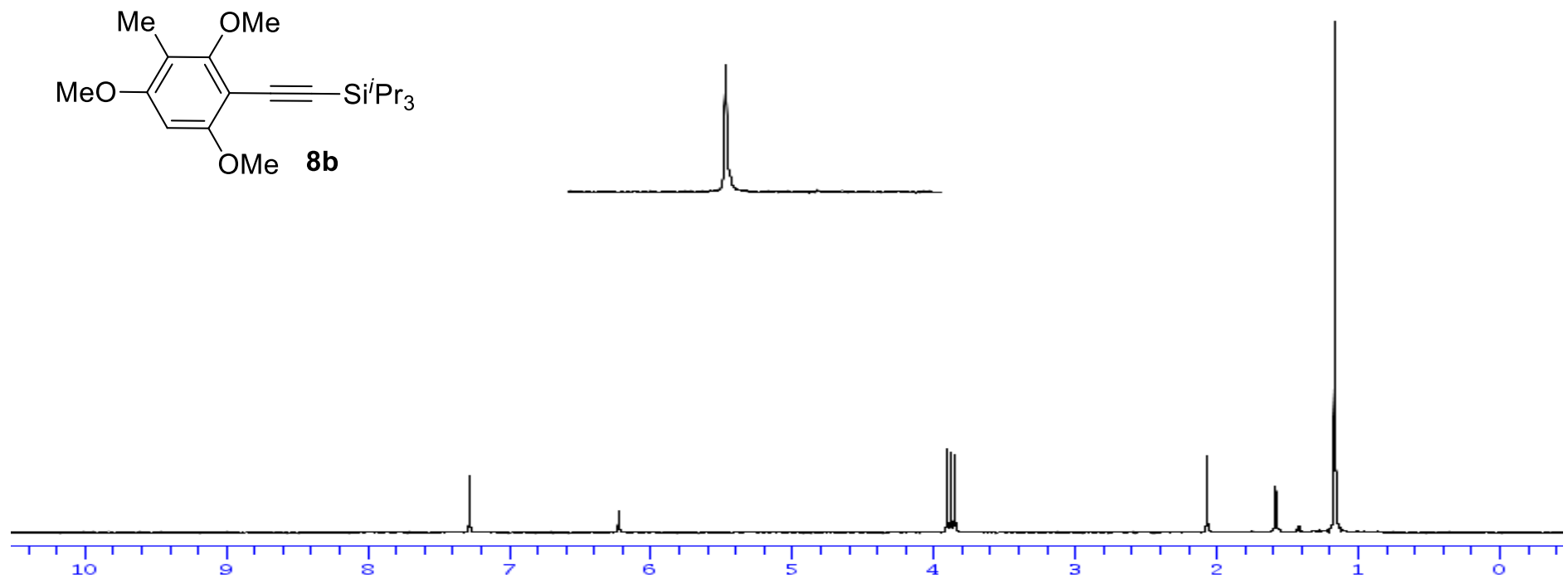

solvent: $<\mathrm{CDCI} 3$

Frequencr:100.612769MHz
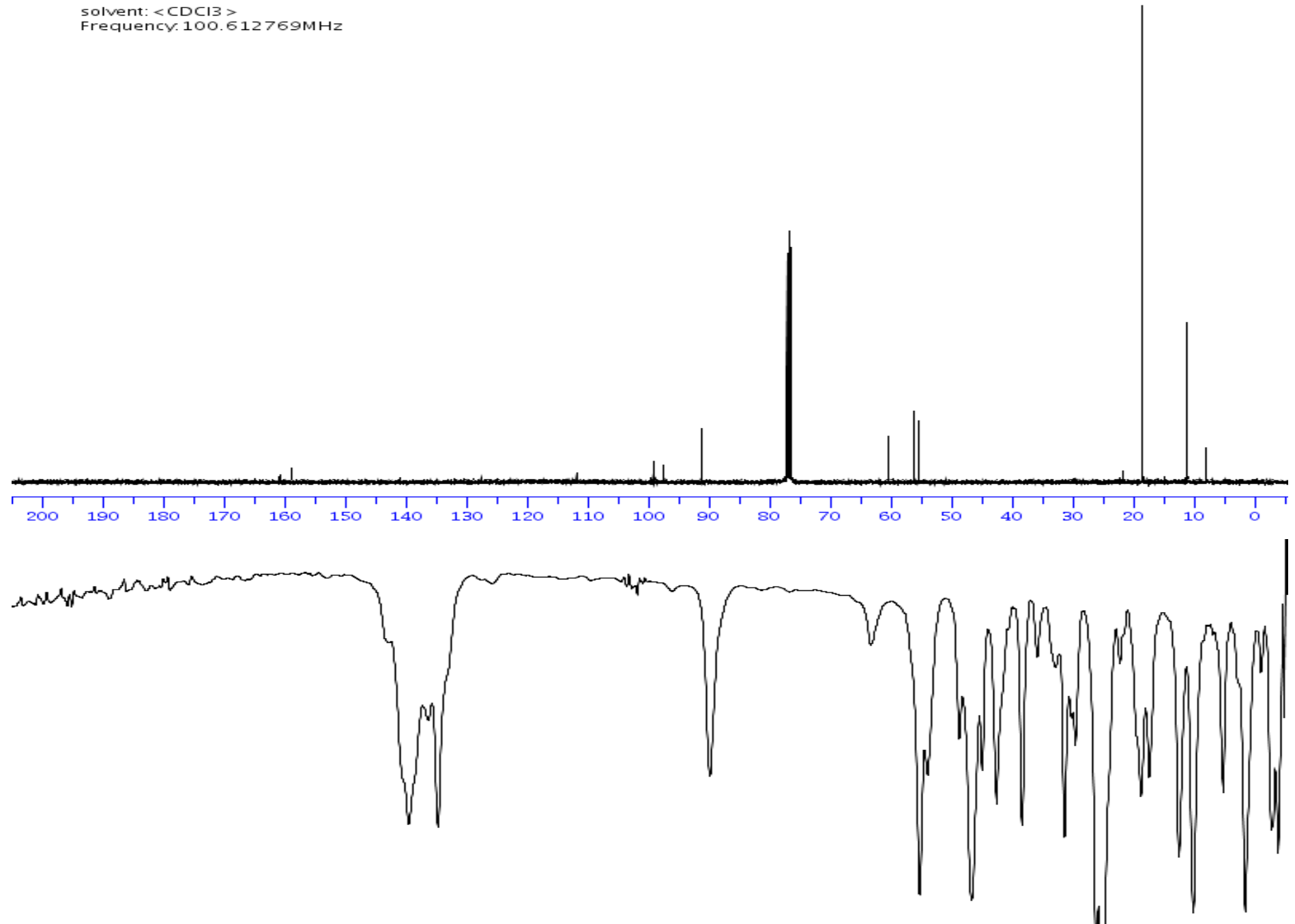